\title{
An Evaluation of the Membership Probability of $212 \lambda$ Boo Stars. I. A Catalogue
}

\author{
Simon J. Murphy ${ }^{1,2,8}$, Christopher J. Corbally ${ }^{3}$, Richard O. Gray ${ }^{4}$, Kwang-Ping Cheng ${ }^{5}$, James E. Neff ${ }^{6}$, \\ Chris Koen ${ }^{7}$, Charles A. Kuehn ${ }^{1,2}$, Ian Newsome ${ }^{4}$ and Quinlin Riggs ${ }^{4}$ \\ ${ }^{1}$ Sydney Institute for Astronomy (SIfA), School of Physics, University of Sydney, NSW 2006, Australia \\ ${ }^{2}$ Stellar Astrophysics Centre, Department of Physics and Astronomy, Aarhus University, 8000 Aarhus C, Denmark \\ ${ }^{3}$ Vatican Observatory Research Group, Steward Observatory, Tucson, AZ 85721-0065, USA \\ ${ }^{4}$ Department of Physics and Astronomy, Appalachian State University, Boone, NC 28608, USA \\ ${ }^{5}$ Department of Physics, California State University, Fullerton, CA, USA \\ ${ }^{6}$ Department of Physics and Astronomy, College of Charleston, Charleston, SC, USA \\ ${ }^{7}$ Department of Statistics, University of the Western Cape, Private Bag X17, Bellville, 7535 Cape, South Africa \\ ${ }^{8}$ Email: murphy@physics.usyd.edu.au
}

(Received July 06, 2015; AcCEPTED August 14, 2015)

\begin{abstract}
The literature on the $\lambda$ Boo stars has grown to become somewhat heterogenous, as different authors have applied different criteria across the UV, optical, and infrared regions to determine the membership status of $\lambda$ Boo candidates. We aim to clear up the confusion by consulting the literature on 212 objects that have been considered as $\lambda$ Boo candidates, and subsequently evaluating the evidence in favour of their admission to the $\lambda$ Boo class. We obtained new spectra of $\sim 90$ of these candidates and classified them on the MK system to aid in the membership evaluations. The re-evaluation of the 212 objects resulted in 64 members and 103 non-members of the $\lambda$ Boo class, with a further 45 stars for which membership status is unclear. We suggest observations for each of the stars in the latter category that will allow them to be confidently included or rejected from the class. Our reclassification facilitates homogenous analysis on group members, and represents the largest collection of confirmed $\lambda$ Boo stars known.
\end{abstract}

Keywords: stars: chemically peculiar, stars: early-type, stars: abundances

\section{INTRODUCTION}

\subsection{The class of $\lambda$ Boo stars}

The $\lambda$ Boo stars are a rare class, making up about $2 \%$ of the population of stars of spectral type A. Their main characteristic is a surface depletion of refractory elements; typically, Fe-peak elements are underabundant by $1 \mathrm{dex}$, and in extreme $\lambda$ Boo stars by 2 dex, while the volatile elements $(C$, $\mathrm{N}, \mathrm{O}, \mathrm{S}$ ) have solar abundances. For many years, this abundance pattern remained unexplained, but its origin is believed to lie in the accretion of material from either the interstellar medium (Kamp \& Paunzen 2002) or a circumstellar disk (Venn \& Lambert 1990; King 1994). In this environment, dust-gas separation occurs, with the refractory elements precipitating into dust grains. The strong radiation field of the A star ejects the dust whilst the star accretes the gas, leaving an enhancement of volatiles compared to refractories on the stellar surface.
Observationally, $\lambda$ Boo stars lie on the main sequence or in the later pre-main-sequence phases. Their calculated ages show a spread throughout the main-sequence phase (Iliev \& Barzova 1995) and some pre-main-sequence stars have $\lambda$ Boo-like abundance patterns (Folsom et al. 2012). Late-A stars fall inside the classical instability strip, where pulsation is driven by the opacity mechanism operating on helium. Hence, it is no surprise that so many $\lambda$ Boo stars with late-A hydrogen line types are found to pulsate (e.g. Weiss et al. 1994; Paunzen et al. 2002b). Asteroseismology of these stars facilitates distinction between a star whose metal deficiencies are limited to the surface, i.e. a $\lambda$ Boo star, and a star that is metal-weak throughout (e.g. Murphy et al. 2013). Asteroseismology may also constrain their ages (Moya et al. 2010; Sódor et al. 2014).

The spectra of $\lambda$ Boo stars show Ca II $\mathrm{K}$ line and metal-line types that are much earlier than the hydrogen line type, which is indicative of the metal weakness. The metal weakness is also apparent in the UV, where the reduced line-blanketing 
leads to a UV excess. UV characteristics of the group were discussed by Faraggiana, Gerbaldi, \& Boehm (1990), and we do not go into detail here. The infrared properties of the $\lambda$ Boo stars have also been investigated (Andrillat, Jaschek, $\&$ Jaschek 1995), and some $\lambda$ Boo stars are known to have an infrared excess (Holweger \& Rentzsch-Holm 1995) that is attributable to the dusty environment in which they reside. The residual dust may play a role in planet formation if the dust is able to coalesce within the strong radiation field. There is some evidence that it can, exemplified by the dusty $\lambda$ Boo star HR 8799 (HD 218396) which has at least four planetary companions (e.g. Soummer et al. 2011). But what is not known is whether the fraction of $\lambda$ Boo stars having an infrared excess is higher than that of normal stars, and an inhibiting factor in ascertaining that ratio is the heterogeneity of the $\lambda$ Boo class. This forms the basis of the motivation of this work, which is discussed in Section 1.2.

Unlike common chemical peculiarities seen in A stars, namely those of the Am and Ap stars, the $\lambda$ Boo phenomenon is not associated with slow rotation; on the contrary, the mean $v \sin i$ of the $\lambda$ Boo stars is in accord with the modal value for normal A stars, that being $\sim 165 \mathrm{~km} \mathrm{~s}^{-1}$ (Abt \& Morrell 1995).

\subsection{Motivation for membership evaluations}

Progress in understanding the $\lambda$ Boo stars has been hindered by a somewhat heterogeneous literature. The last catalogue of $\lambda$ Boo stars was assembled by Paunzen (2001), in the form of a list of new and confirmed $\lambda$ Boo stars, though in print that catalogue did not include some stars that were considered 'classic' $\lambda$ Boo stars at that time (e.g. HD 111786). Heterogeneity continued to proliferate because one survey would demand rejection of a star while another would consider it a firm member of the $\lambda$ Boo group. Without a consolidated evaluation, future studies were left unsure how to treat certain 'members'. The extent of the heterogeneity of the $\lambda$ Boo group was articulated by (and actually exacerbated by) Gerbaldi, Faraggiana, \& Lai (2003), after which interest in the $\lambda$ Boo stars dropped. That work has been heavily criticised (Stütz \& Paunzen 2006; Griffin, Gray, \& Corbally 2012) and we discuss it in more detail in Section 1.4.

Therefore, we aim here to provide a consistent, homogenous membership evaluation for every star that has been classified as a $\lambda$ Boo star in the literature, or considered for membership in that group. One must note that surveys for $\lambda$ Boo stars (e.g. Paunzen et al. 2001) will have considered the membership of hundreds of stars, so we only re-evaluate stars that have been called ' $\lambda$ Boo', or stars that an author has felt the need to stress are 'definitely not $\lambda$ Boo', presuming at least some evidence to the contrary has existed. We summarise the evidence for and against $\lambda$ Boo membership for each of the 212 stars considered as $\lambda$ Boo stars in the literature. We have avoided the inclusion of unpublished $\lambda$ Boo candidates in order to uphold authenticity; a key exception is a dynamic list of $\lambda$ Boo stars kept on Gray's website ${ }^{1}$, as it was when our candidate list was frozen for analysis at the start of 2014.

We thus provide a snapshot of the state of the field at the start of 2014, which will act as a firm footing on which to continue investigations into $\lambda$ Boo stars. In order to maintain as accurate a compilation as possible, we solicit notifications regarding evidence we may have overlooked, in case of future editions.

\subsection{Criteria for membership evaluations}

We considered the following forms of evidence when evaluating the membership status of $\lambda$ Boo candidates.

First and foremost, we look for a spectral classification of ' $\lambda$ Boo', particularly if performed by Gray or Gray \& Corbally, who have been authorities on the classification of $\lambda$ Boo stars for the last 25 years (Gray 1988; Gray \& Corbally 1993) and equally for A stars as a whole (Gray \& Garrison 1987, 1989a, 1989b; Gray \& Corbally 2009). We take a conservative approach to $\lambda$ Boo classifications made by Abt (Abt 1984a, 1985; Abt \& Morrell 1995), who was a little lenient when admitting stars to the $\lambda$ Boo group, having based some inclusions on a weak $\mathrm{Mg}$ II 4481 line only, when in fact that star could have belonged to any of a wider set of groups with that characteristic, such as classical shell stars, field horizontal branch (FHB) stars, or generally metal-weak stars (see Griffin et al. 2012).

We consider photometric measurements, especially Strömgren and $\Delta a$ (Maitzen \& Pavlovski 1989b, 1989a), that might support an optical assessment. A review of the utility of $\Delta a$ photometry in detecting chemically peculiar stars, including $\lambda$ Boo stars, was written by Paunzen, Stütz, $\&$ Maitzen (2005), who concluded that this photometric system is highly efficient in selecting $\lambda$ Boo stars. The use of Geneva photometry for the same purpose was discussed therein, but we deemed there was insufficient added value in this photometric system when compared with Strömgren and $\Delta a$ for it to warrant application here.

Local thermodynamic equilibrium (LTE) abundance analyses of refractory elements, especially iron-peak elements plus silicon and magnesium, are weighted strongly in our evaluations. Accompanying non-LTE (NLTE) abundance analyses for the volatile elements help to establish that the star exhibits the $\lambda$ Boo phenomenon, rather than just generic metal weakness, hence NLTE analyses are highly sought after. An abundance analysis that supports the $\lambda$ Boo spectral classification is the definitive assessment of the $\lambda$ Boo phenomenon, but for a rapidly rotating star the difficulty in performing an abundance analysis restricts the availability of the former somewhat.

The UV character of $\lambda$ Boo candidates provides valuable evidence for or against their inclusion in the class. We mostly use classifications from Baschek et al. (1984) and from Faraggiana et al. (1990), where the latter established their own

\footnotetext{
${ }^{1}$ http://www1.appstate.edu/dept/physics/spectrum/lamboo.txt
} 
criteria for examining the UV character of $\lambda$ Boo stars. Solano $\&$ Paunzen $(1998,1999)$ added to those criteria, but did not re-evaluate $\lambda$ Boo candidates from the literature. In this work, we have also inspected the UV spectra of some stars that were not included in the aforementioned samples, but no detailed analysis of those was attempted. We will refine the UV criteria and conduct a uniform analysis of the UV spectra in a later paper.

We consider an infrared excess or circumstellar absorption in the optical as supporting evidence for membership, but with a low weighting, to avoid contaminating observational results with the expectation that $\lambda$ Boo stars should be dusty if they accrete circumstellar material. Also as a low priority, we note inclusion, or equally, lack of exclusion, from earlier $\lambda$ Boo catalogues.

We use parallax and proper motion measurements from the SIMBAD data base ${ }^{2}$ to calculate a transverse velocity, by converting angular motion at a known distance into a space velocity, so that Population II stars might be identified and rejected. This is more useful than the sole use of proper motions, since nearby objects can have large proper motions without correspondingly large space velocities. We do note, however, that intermediate Pop. II stars cannot be distinguished from $\lambda$ Boo stars based on space velocities alone (Paunzen et al. 2014b).

A summary of the evidence for each of the 212 stars is provided in Section 2. A membership recommendation was then made based on this evidence, as consistently as possible, into one of four classes: member, probable member, uncertain member, or non-member. Notice we have included two categories of 'grey area'. These are used when evidence is in disagreement, inconclusive, or insufficient to make a firm decision. The need for two classes of grey area is founded. For example, some stars were suggested as $\lambda$ Boo stars on very little evidence, and are awaiting verification or nullification. These would fall in the 'uncertain member' group. On the other hand, a star that meets all but one of the criteria for a $\lambda$ Boo star that we have adopted might be considered a 'probable member', if that unsatisfied criterion were a normal UV spectrum, for instance. It is possible that the reader will disagree with some of our recommendations; the categorisation is unavoidably subjective at some level. Our decision-making process is kept transparent with the provision of all of the information involved in that decision. Hence, the reader may also inform his or her own opinion and come to his or her own decision on the borderline cases.

Elemental abundances form a continuous scale between normal and $\lambda$ Boo, hence mild members of the class exist. Where we are persuaded to accept a star as a mild or marginal $\lambda$ Boo, we indicate thusly, so as to distinguish between a definite mild $\lambda$ Boo star and an uncertain member of the class, whose uncertainty arises from conflicting evidence (e.g. a $\lambda$ Boo character in the UV, yet an apparently normal optical spectrum).

\footnotetext{
${ }^{2}$ http://SIMBAD.u-strasbg.fr/SIMBAD/
}

Particularly for stars in the 'uncertain' and 'probable member' categories, we have suggested observations that would help to arbitrate the membership of those stars in the $\lambda$ Boo class. While high-resolution spectra and abundances for every object would be ideal, they are not necessary in all cases, and cannot always be obtained (e.g. where $v \sin i$ is too high). Thus, we recommend full abundance analyses only when the existing evidence is insufficient. In any case, the first step in spectroscopic analyses should be an MK classification (Gray 2014), and so where practical and necessary we obtained at least one spectrum from one or more of the following observatories: Dark Sky Observatory (DSO), the Vatican Advanced Technology Telescope (VATT), Siding Spring Observatory (SSO) or the South African Astronomical Observatory (SAAO).

\subsubsection{Spectroscopic observations and MK classification}

The VATT spectra were obtained over four runs with the VATTspec spectrograph on the VATT $(1.8 \mathrm{~m}$, located on Mount Graham, Arizona). For these observations, the VATTspec is used with a $600 \mathrm{~g} \mathrm{~mm}^{-1}$ grating, which gives a resolution of $1.5 \AA / 2$ pixels in the vicinity of the Ca II $\mathrm{H}$ and $\mathrm{K}$ lines, with a spectral range of $3700-5540 \AA$ A. The spectra are recorded on a low-noise STA0520A CCD with $2688 \times 512$ pixels (University of Arizona Imaging Technology serial number 8228). Three pencil-style lamps, $\mathrm{Hg}, \mathrm{Ar}$, and $\mathrm{Ne}$, were observed simultaneously for wavelength calibrations, and the spectroscopic data were reduced with IRAF using standard techniques.

Many of our spectra were obtained at DSO on the 0.8$\mathrm{m}$ telescope as part of a regular observing programme. The observations were made with the GM spectrograph ${ }^{3}$ using the $1200 \mathrm{~g} \mathrm{~mm}^{-1}$ grating in the first order. The spectra have $1.8 \AA / 2$-pixel resolution, and cover the spectral range 3800 $4600 \AA$ A. A hollow-cathode $\mathrm{Fe}-\mathrm{Ar}$ comparison lamp was used for wavelength calibrations. Raw spectra were reduced using standard IRAF procedures.

Our SAAO spectra were obtained with the Grating Spectrograph on the 1.9-m telescope. The spectra cover the wavelength range $3800-5400 \AA$, and wavelength calibration is performed using a Cu/Ar lamp. The spectral resolution is $2 \AA / 2$ pixel.

Spectra from SSO were obtained during a single run in 2014 April. We used the WiFeS spectrograph (Dopita et al. 2007) on the ANU 2.3-m telescope at SSO. Our spectra in the blue-violet region were obtained in B3000 mode, and have a resolution of about $2.5 \AA / 2$ pixels. The WiFeS data were reduced with the PYwIFEs software package (Childress et al. 2014). Due to difficulty in rectifying the spectra over the Balmer jump, we trimmed the spectra to the range 3865$4960 \AA$. The spectra thus cover the region between the blue wing of $\mathrm{H} 8$ and the red wing of $\mathrm{H} \beta$.

We classified these spectra on the MK system. This enabled many of the initial 'probable' and 'uncertain' members

\footnotetext{
${ }^{3}$ http://www.appstate.edu/ $\sim$ grayro/spectrum/GM/GM.html
} 
to be moved to the 'member' or 'non-member' categories. Each spectrum was classified independently by all three of SJM, CJC and ROG, and after comparison of the initial classifications, the final spectral type assigned was agreed upon by iterative reclassification.

\subsection{Supplementary notes}

\subsubsection{Spectral range}

At early spectral types (at A0 or B9), it is very difficult to be sure of a $\lambda$ Boo classification, because metal lines are nearly absent anyway. Rapid rotation exacerbates the difficulty. The most notable metal lines at these spectral types are the Ca II $\mathrm{K}$ line and $\mathrm{Mg}$ II 4481 . Yet a weak $\mathrm{K}$ line or weak $\lambda 4481$ line alone cannot confirm a $\lambda$ Boo classification, since Am (or hot Am) stars can also have weak $\mathrm{K}$ lines, and shell stars have some features in common with $\lambda$ Boo stars. Good reference lines of iron, e.g. Fe I 4383, are required for comparison with $\lambda 4481$, and these are weak in late B stars. On the other hand, at the hot boundary, $\mathrm{He}$ I lines provide additional constraints on the temperature type (and to a lesser extent, the luminosity type), allowing the strengths of the metal lines to be evaluated from a firmer footing. In practice, we find that a $\lambda$ Boo classification can be considered at spectral types as early as A0.

At later spectral types, i.e. for the F stars, different complications obfuscate the classification. One example is the population of FHB stars. The metal weakness seen in FHB stars cannot be adequately distinguished from those of $\lambda$ Boo stars, unless abundances for the volatile elements $\mathrm{C}, \mathrm{N}$, and $\mathrm{O}$ can be measured. While volatile elements are roughly solar in abundance for $\lambda$ Boo stars, they share underabundances similar to the refractory elements in FHB stars (see, e.g. Takeda \& Sadakane 1997). As such, without the abundances of volatile elements, stars exhibiting $\lambda$ Boo features and having hydrogen line types of $\mathrm{F} 1$ or later are usually called 'late $\lambda$ Boo candidates' and their spectral types are assigned with uncertainty, e.g. 'F2 V kA5mA5 $\lambda$ Boo?'. Unfortunately, access to lines of the desired volatile elements in the classical blue-violet spectral region is poor, and UV or red spectra are required for this. Another difficulty in early $\mathrm{F}$ stars is that the $\mathrm{Mg}$ II 4481 line becomes increasingly blended with $\mathrm{Fe}$, rendering line ratios of, e.g. $\lambda 4481$ to Fe I 4383 , less useful.

\subsubsection{Ages of $\lambda$ Boo stars}

Discussions of $\lambda$ Boo candidates in the literature are not only limited to properties that establish their membership in the class. As an example, the age of $\lambda$ Boo stars was uncertain for a long time, in that modellers did not know whether to use main-sequence or pre-main-sequence evolutionary tracks for them. We do not comment on the age of individual stars since this does not aid the arbitration of membership within the $\lambda$ Boo group, but interested readers can refer to the work of Iliev \& Barzova (1995), who determined ages for definite $\lambda$ Boo stars (as classified here and elsewhere), and the review in the introduction of Paunzen et al. (2002a) along with ref- erences therein. It has been shown that $\lambda$ Boo stars have ages spanning from the zero- to the terminal-age main sequence (ZAMS and TAMS, respectively), and HAeBe stars identified as $\lambda$ Boo stars almost certainly extend that range to some of the pre-main-sequence phase. For convenience, we make note of the availability (typically from Iliev \& Barzova 1995) of 'key physical parameters', which include mass, effective temperature, radius, surface gravity, luminosity, and age.

\subsubsection{Pulsation in $\lambda$ Boo stars}

The pulsational properties of some candidates have also been presented by various authors. In favourable circumstances, asteroseismology can distinguish between global and surface depletions of metals. The requirement of excellent asteroseismic data has led to only a few determinations being made in this way. The available pulsational information is generally insufficient for arbitrating membership in the $\lambda$ Boo class, and not all stars have been monitored for oscillations, hence we have not generally included this information in our descriptions in Section 2. We leave the assessment of pulsations as future work. This document hence serves as a platform for future, homogeneous studies.

\subsubsection{Stellar rotation}

Rotation is an important property, in that rotation broadens spectral lines. At classification resolution (1.8 A per 2 pixel, or $\mathrm{R} \sim 2500$ at $4500 \AA$ ), a very fast rotator having $v \sin i>$ $200 \mathrm{~km} \mathrm{~s}^{-1}$ can mimic a $\lambda$ Boo star because the metal lines become broader and shallower. If they are sufficiently broad, they can blend and appear to be part of the continuum, and hence the star appears metal weak or $\lambda$ Boo-like. One must be very cautious in accepting a $\lambda$ Boo star if it has been noted to have a very high $v \sin i$. In Section 3, we tabulate the rotational velocity for $\lambda$ Boo candidates for which it has been measured.

\subsubsection{Binarity and the 'composite spectrum hypothesis'}

A discussion of binarity among $\lambda$ Boo stars warrants a short digression. At the turn of the century, a large amount of effort was being put into finding new $\lambda$ Boo stars while a complete understanding of the origin of their abundance anomalies was still lacking. In a series of papers, Faraggiana, Bonifacio and Gerbaldi attempted to demonstrate that the $\lambda$ Boo stars were a 'non-homogeneous' group and that a large number of them could be shown to be binaries (Faraggiana \& Bonifacio 1999; Faraggiana et al. 2001b; Faraggiana, Gerbaldi, \& Bonifacio 2001a; Faraggiana \& Gerbaldi 2003; Gerbaldi \& Faraggiana 2004). Their suggestion that the class was heterogeneous was in disagreement with Paunzen et al. (2002b), who showed the class to be quite homogeneous. Furthermore, the insistence that binaries be dropped from the class was in direct contradiction with the results of Iliev et al. (2001, 2002) who showed that for two separate $\lambda$ Boo binary systems, each member of each system (that is, all four stars) could be demonstrated to be true $\lambda$ Boo stars. Given the importance of binary systems in providing fundamental stellar 
parameters such as mass and radius, the suggestion to drop them would only hinder progress in $\lambda$ Boo star research.

Still, the 'binary hypothesis' - that binarity was responsible for mimicking metal weakness-was pursued. Faraggiana \& Bonifacio (2005) demonstrated that the characteristic solar abundance of $\mathrm{C}, \mathrm{N}$, and $\mathrm{O}$ in $\lambda$ Boo stars arises naturally if a binary system is confused as a single star and labelled a $\lambda$ Boo star. Conversely, Stütz \& Paunzen (2006) synthesised 105 hypothetical binary systems and compared the synthesised spectra with those of known $\lambda$ Boo stars. Their results indicated that for about 90 per cent of the group members, the spectroscopic binary hypothesis could not explain the observations.

The binary hypothesis requires that the radial velocity difference between the two stars in the binary causes the continuum of one star to 'veil' the lines of the other, implying that the radial velocity difference should be comparable to the $v \sin i$ of the stars. Griffin et al. (2012) sought to test the veiling mechanism and found it could produce the appearance of metal weakness by only a factor of about 2 , i.e. $[M / \mathrm{H}]=-0.3$ dex; it certainly could not produce metal weaknesses on the order of -2.0 dex that is seen in some $\lambda$ Boo stars.

Furthermore, Faraggiana et al. (2004) declared many stars to have 'composite spectra' based on variable radial velocities, i.e. by association in an SB 1 system. Their variable radial velocities were taken from comments of $V$ or $V$ ? from the Bright Star Catalogue (BSC; Warren \& Hoffleit 1987). Griffin et al. (2012) demonstrated that out of a sample of 12 stars that were $\lambda$ Boo candidates and were classified as having variable RVs in the BSC, 11 did not have any appreciable RV variability in their long-term monitoring programme. Further, they found that RV measurements of A stars 'can depend on the spectral region as well as on the technique adopted and the quality of the spectrum.' This work abolished the composite spectrum binary hypothesis. We also note that the radial velocity variations of a pulsating ( $\delta \mathrm{Sct})$ star can reach several $\mathrm{km} \mathrm{s}^{-1}$ (Breger, Hutchins, \& Kuhi 1976), and that the $\lambda$ Boo stars are predicted to be less stable against pulsation than normal A stars of the same spectral type (Murphy 2014).

In conclusion, we do not consider binarity to be a good reason to drop a star from the $\lambda$ Boo class, especially if the binarity is classified in the form of a 'variable radial velocity' or 'SB1' system. Since in A-star spectra it is not easy to distinguish between genuine line-doubling and rotational broadening if the rotational velocity is high-around $120 \mathrm{~km} \mathrm{~s}^{-1}$ or more-only 'SB2' classifications of binarity are meaningful in this regard, and only then when they have been determined with spectra of high resolution and high signal to noise.

Nonetheless, it is important to be aware of close companions (closer than 2 arcsec) that may contaminate CCD photometry or spectroscopy. We have therefore checked each candidate for close companions in the Washington Double Star (WDS) catalogue (Mason et al. 2001), and noted any matches. The Catalogue of Components of Double and Mul- tiple stars (CCDM, Dommanget \& Nys 2002) was also used for confirmation.

\subsection{Document layout}

In Section 2, we evaluate the membership of each $\lambda$ Boo candidate. The format of Section 2 is a list of stars ordered by increasing HD number, with one eponymous subsection per $\mathrm{star}^{4}$, in which the $\lambda$ Boo status of that star is investigated. New observations are recorded individually there. In Section 3 , we summarise the properties of the candidates and the recommendations made for them. A reference list is provided at the end.

\section{NOTES ON MEMBERS OF THE $\lambda$ BOO CLASS}

\subsection{HD 3}

Abt \& Morrell (1995) classified this star as A0 Vn(Lam Boo) with $v \sin i=210 \mathrm{~km} \mathrm{~s}^{-1}$, raising the concern this might just be a rapid rotator. There is little support for $\lambda$ Boo membership in the literature. Spectrum required. Recommendation: uncertain member.

\subsection{HD 319 (HR 12)}

Identified as a $\lambda$ Boo star by Abt (1984a), and given the spectral type A1mA2 Vb $\lambda$ Boo by Gray (1988). Gray's comments were that ' $\mathrm{Ca}$ II $\mathrm{K}$ has the strength of an A1 star, the metallic line spectrum that of an A2 star... Hydrogen lines show peculiar profiles with cores having strengths like those of an A7 star, but with a broader, shallower profile, and extraordinarily broad but shallow wings'. As he also noted, HD 319 has a common-proper-motion companion 1.9 arcsec away that is 5.1 mag fainter (WDS, Mason et al. 2001). The star falls mildly among the $\lambda$ Boo group (away from the line of normal stars) in the Maitzen \& Pavlovski (1989a) $\Delta a$ photometry study. Accepted as a $\lambda$ Boo star in the Renson, Faraggiana, \& Boehm (1990) catalogue. Stürenburg's (1993) abundance analysis shows a clear $\lambda$ Boo pattern, with a 0.75-dex underabundance of metals. Key physical parameters are available (Iliev \& Barzova 1995). Holweger \& Rentzsch-Holm (1995) found no signature of circumstellar material around the $\mathrm{Ca}$ II $\mathrm{K}$ line of this star. Paunzen et al. (1999b) confirmed near-solar $[\mathrm{C} / \mathrm{H}]$ and $[\mathrm{O} / \mathrm{H}]$ NLTE abundances. Faraggiana et al. (2004) recorded this star as a suspected double star, based on the aforementioned common-proper-motion companion, but in reality the separation and magnitude difference are too great to cause a false positive $\lambda$ Boo detection. Recommendation: member.

\footnotetext{
${ }^{4} \lambda$ Boo candidates commonly known under other names have those names appended to the subsection title in parentheses.
} 


\subsection{HD 2904}

Classified as A0 Vnn(Lam Boo) by Abt \& Morrell (1995), with $v \sin i=225 \mathrm{~km} \mathrm{~s}^{-1}$. The rapid rotation calls into question the $\lambda$ Boo classification. Hauck, Ballereau, \& Chauville (1998) found interstellar Ca II $\mathrm{K}$ line absorption, but there is no real evidence in favour of membership into the $\lambda$ Boo class. Spectrum required. Recommendation: uncertain member.

\subsection{HD 4158}

Long-known $\lambda$ Boo star (Graham \& Slettebak 1973). The Maitzen \& Pavlovski (1989a) $\Delta a$ photometry study gives a strong indication that this is a $\lambda$ Boo star, which agrees well with a UV assessment (Faraggiana et al. 1990). We have also inspected the IUE (International Ultraviolet Explorer) spectrum available for this star. There is not enough flux at $1600 \AA$ to see any depression, and the $1657 / 1670$ ratio also suffers from lack of flux; at longer wavelengths the overall character is metal weak, but the $\lambda 1937 \mathrm{C}$ line is strong. This is consistent with a $\lambda$ Boo classification. $[\mathrm{C}]$ abundance is -0.16 (Paunzen et al. 1999b). We classified a new spectrum of this star as F2 V kA1mA1 $\lambda$ Boo?, with the comment that Mg II 4481 is almost absent. Given the UV characteristics and near-solar $\mathrm{C}$ abundance, we can accept this as a $\lambda$ Boo star. Recommendation: member.

\subsection{HD 5789}

Hauck et al. (1998) found interstellar Na I D lines and Ca II K line absorption. Abt \& Morrell (1995) classified the star as B9.5 Vnn(Lam Boo); the 'nn' classification raises concerns. Nevertheless, Gerbaldi et al. (2003) found no reason to reject the star, even though it is a spectroscopic binary and has $v \sin i=300 \mathrm{~km} \mathrm{~s}^{-1}$ (Slettebak 1963). The evidence favours exclusion. Recommendation: non-member.

\subsection{HD 6173}

Among the $\lambda$ Boo population in the Maitzen \& Pavlovski (1989b) $\Delta a$ photometry study. Listed as a $\lambda$ Boo star in the Renson et al. (1990) catalogue. Gerbaldi et al. (2003) found no reason to reject this star. Classification of A0 Vn by Abt (1984b) and A0 IIIn by Paunzen et al. (2001). Photometry suggests a late A star (roughly A7) according to both $\beta$ and $b-y$. Even if the star is a giant, $b-y$ is not strongly influenced by luminosity so the agreement suggests $\sim \mathrm{A} 7$. The $c_{1}$ index is high for a typical A7 star. It is at A7 that the $m_{1}$ index reaches its maximum, with a value of $\sim 0.200$ according to Crawford's $(1975,1978,1979)$ standard relations, yet this star has $m_{1}=0.079$ (Hauck \& Mermilliod 1998) which is much weaker than even a typical A0 star. Normally, $m_{1}$ scales with $c_{1}$, so that a giant luminosity class has a higher $m_{1}$ index, hence there is good photometric evidence for metal weakness in HD 6173. $\lambda$ Boo membership is therefore not ruled out but a spectrum is definitely needed. Recommendation: uncertain member.

\subsection{HD 6870}

Identified as a 15-mmag, metal-weak pulsator (Breger 1979), HD 6870 falls among the $\lambda$ Boo population in the Maitzen \& Pavlovski (1989a) $\Delta a$ photometry study. It has a clear $\lambda$ Boo character in the UV (Faraggiana et al. 1990), and is listed as a $\lambda$ Boo star in the Renson et al. (1990) catalogue. $[\mathrm{C} / \mathrm{H}]$ and $[\mathrm{O} / \mathrm{H}]$ are both positive (Paunzen et al. 1999b). Gray's classification for the Paunzen et al. (2001) paper reads: 'HD 6870 (kA2hA7mA2 LB PHL): this star is clearly a lambda Boo'. Gerbaldi et al. (2003) argued this star is misclassified because it has kinematics slightly deviating from those of Pop. I stars, and suggested it belongs the thick disc population instead. Indeed, we note a transverse velocity of $60 \mathrm{~km} \mathrm{~s}^{-1}$, easily putting it in the top decile of stars considered in this work, and note that it is called an SX Phe star and an 'old-disc' star in the older literature. However, the solar $[\mathrm{C} / \mathrm{H}]$ and $[\mathrm{O} / \mathrm{H}]$ argue against a generic metal weakness expected from thick disc stars, and Paunzen et al. (2014b) warn against using velocities in $\lambda$ Boo membership assessments. Paunzen et al. (1999b) found discrepant $v \sin i$ values between the C and $\mathrm{O}$ lines; the lower $\left(128 \mathrm{~km} \mathrm{~s}^{-1}\right)$ agrees with the earlier value of Rodgers $\left(1968,130 \mathrm{~km} \mathrm{~s}^{-1}\right.$ ). Recommendation: member.

\subsection{HD 7908}

Classified as hF0mA3 LB by Paunzen et al. (2001), for which Gray commented that the spectrum was 'clearly $\lambda$ Boo'. It was accepted as a bona fide $\lambda$ Boo star in the results paper of that series (Paunzen 2001). Gerbaldi et al. (2003) found no reason to reject this star from the $\lambda$ Boo class. Recommendation: member.

\subsection{HD 9100}

This star is listed by Abt \& Morrell (1995) as an A2.5 V star (with $v \sin i=110 \mathrm{~km} \mathrm{~s}^{-1}$ ). It had been earlier classified as A3 Vb $\lambda$ Boo PHL? by Gray \& Garrison (1989b). To be specific, those authors said 'The hydrogen lines of this star show very broad, but rather shallow, wings and are reminiscent of the hydrogen lines of the PHL $\lambda$ Boo stars of Gray (1988). The $\beta$ index of this star is surprisingly low for hydrogen lines with such broad wings, a characteristic which also recalls the PHL $\lambda$ Boo stars. The $\lambda 4481$ line is slightly, but not obviously, weak. In the $b-y, m_{1}$ plane, this star lies within the $\lambda$ Boo "distribution". We obtained two spectra of this star with two different instruments, both of which we (independently) classified as A3 IVs (4481wk). In both spectra, the Mg II 4481 line is quite weak, but the other metal lines show weaknesses of no more than half a spectral subclass (i.e. A2.5). This classification differs considerably from the classification of Gray \& Garrison 
(1989b). Could this star be a spectrum variable? It is noteworthy that it is a $\delta$ Sct star with 20-mmag light variation, and was at one time the bluest $\delta$ Sct star known (Breger 1969). There are many papers on its $\delta$ Sct nature. Recommendation: non-member.

\subsection{HD 11413}

First identified by Abt (1984a) and later classified by Gray (1988) as 'A1 Va $\lambda$ Boo', whereupon it was described as having $\mathrm{Ca}$ II $\mathrm{K}$ line and metallic-line types 'intermediate to the A0 and A1 standards, with weak $\lambda 4481$. Hydrogen lines show broad, but rather shallow wings, but peculiarly weak cores, similar to the cores of an F0 star'. This star lies among the $\lambda$ Boo population in the Maitzen \& Pavlovski (1989a) $\Delta a$ photometry study, and appears in the Renson et al. (1990) $\lambda$ Boo catalogue. The abundance analysis carried out by Stürenburg (1993) shows solar $[\mathrm{C} / \mathrm{H}]$ but most metals are between -1.0 and -1.5 dex. Key physical parameters are available (Iliev \& Barzova 1995). Holweger \& Rentzsch-Holm (1995) detected infalling circumstellar gas around this star's Ca II K line. $[\mathrm{C} / \mathrm{H}]$ and $[\mathrm{O} / \mathrm{H}]$ are -0.25 and -0.11 dex, respectively (Paunzen et al. 1999b), which alongside the abundances from Stürenburg (1993) for the metals confirms a $\lambda$ Boo pattern. Koen et al. (2003) conducted a dedicated analysis of the pulsational content and stellar parameters from spectroscopy, which confirmed the star's $\lambda$ Boo nature. Recommendation: member.

\subsection{HD 11502}

HD $11502\left(\gamma^{1}\right.$ Ari) and HD $11503\left(\gamma^{2}\right.$ Ari $)$ are in a double system separated by 8.51 arcsec. The former has been reclassified for this work as A0 IV-V(n) kB8; the latter is an $\alpha^{2} \mathrm{CVn}$ star (and thus not a $\lambda$ Boo star), with spectral type 'knA0hA3 (IV) SiSr' (Gray \& Garrison 1989b). The spectrum of HD 11502 is metal weak except for the $\lambda 4481$ line, and the $\mathrm{H}$ and $\mathrm{He}$ lines confirm the temperature subclass $\mathrm{A} 0$. It is not a $\lambda$ Boo star.

However, confusion has arisen in the literature, as indicated by the fact that an essential note on SIMBAD states that HD 11503 is not HR 545; HR 545 is the designation of HD 11502. Such confusion is exemplified in Abt \& Cardona (1984), and corroborated by the fact that literature values of $v \sin i$ for HD 11503 are wildly discordant, and much too high for an $\alpha^{2} \mathrm{CVn}$ star. Recommendation: non-member.

\subsection{HD 11503}

See entry for HD 11502. Recommendation: non-member.

\subsection{HD 11905}

Misclassified, according to Renson et al. (1990). Classified as B8 III by Cowley et al. (1969), and as B9 HgMn in the Renson \& Manfroid (2009) catalogue. Rejected by Paunzen et al. (1997) for the Ap/Bp classification. Recommendation: non-member.

\subsection{HD 13755}

Paunzen et al. (2001) classified this star as hF2mA5 V LB. Gray's notes to Paunzen for this star read: 'HD 13755 (kA5hF2mA5 V LB): H-lines and metallic-line morphology match the F2 V std quite well. However, metals are clearly weak, at about A5. Hence, clearly metal-weak. hF2mA5 V'. We have observed and classified HD 13755 for this work and give the spectral type F1 V kA9mA6 ( $\lambda$ Boo), as thus accept it as a mild member. An abundance analysis should be performed to confirm membership. Recommendation: member.

\subsection{HD 15164}

See entry for HD 15165. Recommendation: member.

\subsection{HD 15165 (VW Ari)}

We note this is a visual triple system. We give component $\mathrm{A}$ the spectral type $\mathrm{F} 2 \mathrm{~V} \mathrm{kA} 2 \mathrm{~mA} 2 \lambda$ Boo? (i.e. this is a 'late $\lambda$ Boo candidate', see Section 1.4.1). Component B is in SIMBAD as HD 15164, to which we give the spectral type F1 V kA7mA6 ( $\lambda$ Boo)?, and component $\mathrm{C}$ has the designation HD $15165 \mathrm{C}$ that we classify as a normal K2 V star. The stars have proper motions that are the same to within one or two mas $\mathrm{yr}^{-1}$, so they are a physical triple. Paunzen et al. (2002a) did an abundance analysis, but did not specify which component was analysed. We therefore assume it was component $\mathrm{A}$, which is the brightest by two magnitudes. They found that $\mathrm{C}, \mathrm{N}, \mathrm{O}$, and $\mathrm{S}$ are approximately solar while $\mathrm{Mg}, \mathrm{Si}$, and $\mathrm{Ca}$ are 1-dex underabundant, which is a typical $\lambda$ Boo abundance pattern. Andrievsky et al. (1995) published a paper on the composition of components $\mathrm{A}$ and $\mathrm{B}$, which include a 2.2- and a 1.8- $\mathrm{M}_{\odot}$ star. The secondary is described as being of solar composition but the primary has $[\mathrm{Fe} / \mathrm{H}]$ of -0.46 . Their leading hypothesis for the differing composition was stellar capture. The primary, they say, is the $\delta$ Sct (or SX Phe) star and the secondary is non-variable. There are many $\delta$ Sct-themed papers on this star. We disagree that the secondary can have solar abundances given the appearance of the classification spectrum; a self-consistent abundance analysis of all three components, with NLTE abundances for the volatile elements, is highly desired. Recommendation: member.

\subsection{HD 16811}

Hauck et al. (1998) noted Na I absorption for this star, which is of uncertain membership in the Renson et al. (1990) catalogue. The Maitzen \& Pavlovski (1989a) $\Delta a$ photometry study shows it to be rather mild, not lying far from the line of normal stars. Gray \& Garrison (1987) classified it as A0 IVn, 
with the comment 'classified as a marginal $\lambda$ Boo star by Abt (1984). It does not look peculiar in our one (slightly underexposed) spectrum. Occultations indicate this star is a triple system'. Recommendation: non-member.

\subsection{HD 16955}

Very near the line of normal stars in the Maitzen \& Pavlovski (1989a) $\Delta a$ photometry study. Andrillat et al. (1995) stated 'according to all indications, this is a normal star'. A star with circumstellar Ca II K line absorption (Hauck et al. 1998). $u v b y \beta$ observations (Hauck \& Mermilliod 1998) indicate a normal A3 star. Recommendation: non-member.

\subsection{HD 16964}

HD 16964 was recorded as the brighter star in a visual double with HD 16965 and given a spectral type of A1 by Bidelman (1943). HD 16964 was classified as an A0 V $\lambda$ Boo star on a 1.2-mm wide spectrum by Abt (1988) and is clearly designated there as the fainter of the pair, but by only 0.3 mag in V. Their separation is 15.8 arcsec. No details were given on the reason for classification as $\lambda$ Boo, so we have reobserved both members. We give the spectral type A0 IV-V to component $\mathrm{A}$, and A0.5 IVn to component $\mathrm{B}$. The Ca II $\mathrm{K}$ line in component $\mathrm{B}$ is slightly peculiar, but neither component is a $\lambda$ Boo star. Recommendation: non-member.

\subsection{HD 17138 (RZ Cas)}

Narusawa et al. (2006a) determined this eclipsing algol-type binary to have $\lambda$ Boo-like abundances, and Narusawa et al. (2006b) presented evidence suggestive of circumstellar matter in the system. Richards \& Albright (1999) previously observed a gas stream for RZ Cas. The A3 V spectral type of the primary of this system is agreed upon in the literature, the secondary being K0 IV. The abundance analysis of Narusawa et al. (2006a) showed 'definite under-abundances (by -0.45 dex or more) of $\mathrm{Mg}, \mathrm{Si}, \mathrm{Ti}, \mathrm{Cr}$, and $\mathrm{Fe} \ldots$ On the other hand, light elements $\mathrm{C}$ and $\mathrm{O}$ show[ed] solar abundances and $\mathrm{Ca}$ [was] slightly underabundant ( $-0.3 \mathrm{dex})$ '. The mild $\lambda$ Boo abundance pattern favours acceptance, but its existence in a short-period binary $(P=1.1953 \mathrm{~d})$ raises suspicions. The RZ Cas system is well parametrized in, for example, Narusawa et al. (2006a). Recommendation: probable member.

\subsection{HD 21335}

Classified by Gray \& Garrison (1989b) as an A3 IVn star with the comment: 'Metals may be slightly weak, but $\lambda 4481$ is quite normal. Abt (1984) classifies this star as a marginal $\lambda$ Boo star. High $v \sin i$ '. Falls between the true $\lambda$ Boo and the normal stars in the Maitzen \& Pavlovski (1989a) $\Delta a$ photometry study. Tagged 'LB' in the Andrillat et al. (1995) IR study of $\lambda$ Boo stars, with the comment ' $\mathrm{Ca}$ is only slightly deficient', indicating no sign of a shell. Gerbaldi et al. (2003) found no cause for rejection. Likely, a composite spectrum according to Faraggiana et al. (2004) because Speckle interferometry indicated an object separated by less than 0.1 arcsec but with no magnitude difference recorded. The 0.1 arcsec separation is confirmed in the WDS catalogue (Mason et al. 2001). Recommendation: non-member.

\subsection{HD 22470}

Rejected by Gray (1988). Has a mutually exclusive spectral type: B9 Si (Renson \& Manfroid 2009). First appeared in papers on magnetic chemically peculiar stars four decades ago (Landstreet et al. 1975), and its average surface field strength is measured to be $2350 \mathrm{G}$ (Glagolevskij 2011). Has a companion at 0.2 arcsec that is $0.4 \mathrm{mag}$ fainter (WDS, Mason et al. 2001). Recommendation: non-member.

\subsection{HD 23258}

First classified as $\lambda$ Boo by Abt $\&$ Morrell (1995) (A0 Vp(Lam Boo)), but was described as a 'borderline case' by Paunzen \& Gray (1997), ultimately being classified as 'A0 Vb (sl wk metals)'. Paunzen et al. (2001) put it on a list of stars that were 'not classical $\lambda$ Boo stars', but of which some 'may be metal weak'. It did not make the Paunzen (2001) 'new and confirmed' list of $\lambda$ Boo stars. Andrievsky et al. (2002) found 'the typical abundance pattern (C and O solar whereas $\mathrm{Mg}, \mathrm{Si}, \mathrm{Cr}$, and Fe are moderately underabundant)'. Listed as a mild $\lambda$ Boo star on Gray's website, with spectral type A3 V kB9.5mB9.5 (Boo). Recommendation: member.

\subsection{HD 23392}

Presented as a new $\lambda$ Boo star with a spectral type A0 $\mathrm{Va}^{-}$ ( $\lambda$ Boo) by Paunzen \& Gray (1997), with the comment 'mild $\lambda$ Bootis star, hydrogen lines show broad wings'. We reclassified this star for this work, giving the spectral type A0 $\mathrm{Va}^{-} \mathrm{kB} 8.5$ ( $\lambda$ Boo), and note that the He I and H-lines clearly fix the spectral type at A0, but the $\lambda 4481$ and $\mathrm{K}$ line are weak. Photometrically constant at the 2-mmag limit (Paunzen et al. 1998c). Gerbaldi et al. (2003) called this star's UV flux 'inconsistent' because it was too high for them to fit, and included the note 'bin?', implying they suspected the star of binarity, but Marchetti, Faraggiana, \& Bonifacio (2001) found no evidence of binarity in their speckle interferometry survey. Recommendation: member.

\subsection{HD 24712}

Misclassified according to Renson et al. (1990). Recorded as an $\alpha^{2}$ CVn star on SIMBAD, with spectral type 'A9 Vp SrEuCr' (Abt \& Morrell 1995), necessitating rejection. Recommendation: non-member. 


\subsection{HD 24472}

Classified as 'hF2mA5 V LB' (Paunzen et al. 2001; Paunzen 2001). Gerbaldi et al. (2003) noted 'inconsistent UV flux (bin?)'. An uncertain late $\lambda$ Boo star on Gray's website, Gray (1989) has previously classified it as F3 V m-2, and in his notes to Paunzen on the aforementioned classification, Gray wrote 'Is it a Lambda Boo, or a Pop. II?' NLTE abundances of volatile elements would be required to confirm. Recommendation: probable member.

\subsection{HD 26801}

An uncertain member of the class according to the Renson et al. (1990) catalogue, owing to a classification by Abt (1985) of 'A0 III lambda Boo?'. The 'doubtful' infrared IRAS excess described by King (1994) is most likely associated with a neighbour at 38 arcsec. No strong support for $\lambda$ Boo membership in the literature. Recommendation: non-member.

\subsection{HD 27404}

Appears in Hauck's (1986) 'Search for $\lambda$ Boo candidates' paper, but is listed with a classification of Ap Si. Renson et al. (1990) rejected this star from their catalogue. Renson \& Manfroid (2009) gave a spectral type A0 Si. It is a known magnetic chemically peculiar star, with longitudinal field strength on the order of a kG (Kudryavtsev et al. 2006). Definitely not a $\lambda$ Boo star. Recommendation: non-member.

\subsection{HD 30422}

First identified as a $\lambda$ Boo star by Gray \& Garrison (1989b), with the spectral type A3 $\mathrm{Vb} \lambda$ Boo. Key physical parameters are available (Iliev \& Barzova 1995). NLTE abundances for $[\mathrm{C} / \mathrm{H}]$ and $[\mathrm{O} / \mathrm{H}]$ are -0.27 and -0.25 , respectively (Paunzen et al. 1999b). Gerbaldi et al. (2003) found no cause for rejection, but Faraggiana et al. (2004) called it a 'probable double' implying a composite spectrum, based on the radial velocities of Grenier et al. (1999); we see no evidence for binarity there, and note that only 'SB2' would be convincing evidence of binary contamination. We reobserved this target and classify it as A7 V kA3mA3 ( $\lambda$ Boo). Recommendation: member.

\subsection{HD 30739}

Spectral type of A0 Vp(Boo)n in Abt \& Morrell (1995). Comment of 'inconsistent UV flux (solar ab.)' in Gerbaldi et al. (2003). Classified as a normal star (A0.5 IVn) by Gray $\&$ Garrison (1987). No real evidence in favour of $\lambda$ Boo membership. Recommendation: non-member.

\subsection{HD 31293}

Gray \& Corbally (1998) classified HD 31293 (AB Aur) as 'A0 Vaer $\mathrm{Bd}<\mathrm{Nem5}$ ' but made no mention of $\lambda$ Boo properties and were explicitly looking for young $\lambda$ Boo stars. Identified by Acke \& Waelkens (2004) to have deficient Fe and enhanced $\mathrm{N}$ and $\mathrm{O}$. Folsom et al. (2012) called the Acke \& Waelkens (2004) abundance analysis into question, by pointing out the remarkably high $\log g$ value of 5.0 that was used. Folsom et al. (2012) favoured a more solar-like Fe abundance. They also provided key physical parameters. The star is a dusty HAeBe star, with an unconfirmed planetary system (Oppenheimer et al. 2008; Hashimoto et al. 2011). We have eight spectra, and the Mg II 4481 line is prominent in all of them. It is a spectrum variable, but at no phase does it appear like a $\lambda$ Boo star. In one of our spectra, it looks like a shell star, whereas in the others the hydrogen lines are in emission. Recommendation: nonmember.

\subsection{HD 31295}

One of the first $\lambda$ Boo stars identified (Slettebak 1954). Shown to have small space velocities (Hauck \& Slettebak 1983), and particularly strong $\lambda$ Boo characteristics in the UV (Baschek et al. 1984)-equivalent to or stronger than $\lambda$ Boo itself. The UV properties were later confirmed by Faraggiana et al. (1990). Sadakane \& Nishida (1986) reported a 60- $\mu \mathrm{m}$ excess, and included it in a list of Vega-like stars, implying the presence of cool circumstellar material. Gray (1988) included it in his list of $\lambda$ Boo stars, with the spectral type A0 Va $\lambda$ Boo, and the comment: "Very weak $\mathrm{K}$ line and $\lambda 4481$. Hydrogen lines are strong, like those of an early A type dwarf, and show broad, well-developed wings. Weak metallic lines are visible at [Fe I] $\lambda 4046$ and [Fe I] $\lambda 4064$ '. In this work, we reobserved the star and give the classification $\mathrm{kA} 0 \mathrm{hA} 3 \mathrm{~mA} 0 \mathrm{Va}^{-} \lambda$ Boo. HD 31295 falls mildly off the line of normal stars (towards $\lambda$ Boo stars) in the Maitzen $\&$ Pavlovski (1989b) $\Delta a$ photometry study. Accepted as a definite member of the $\lambda$ Boo class in the Renson et al. (1990) catalogue. Iliev \& Barzova (1993b) inspected the hydrogen lines of this and other $\lambda$ Boo stars, and proclaimed HD 31295 is an 'excellent example of a $\lambda$ Bootis type star with normal hydrogen lines'. Stürenburg (1993) confirmed a $\lambda$ Boo abundance pattern, with -1-dex metals, which was supported by later NLTE abundance analyses that showed almost solar C, N, O, and S (Paunzen et al. 1999b; Kamp et al. 2001) and by an LTE analysis of metals (Paunzen et al. 1999a). Key physical parameters are available (Iliev \& Barzova 1995). Gerbaldi et al. (2003) found no reason to reject this star, but Faraggiana et al. (2004) called it a probable double based on a variable $\mathrm{RV}$ that could not be traced in the cited text. This is one of the best-studied, bona fide $\lambda$ Boo stars. Recommendation: member. 


\subsection{HD 34787}

Paunzen et al. (1997) rejected HD 34787 because it did not meet the UV criteria of $\lambda$ Boo stars in the IUE analyses by Baschek et al. (1984) and Faraggiana et al. (1990). However, according to Gerbaldi et al. (2003), HD34787 was never mentioned in either of those two papers and has never been observed by the IUE satellite. Thus, Gerbaldi et al. (2003) rejected this star due to former misclassification, whereas they should have reobserved and/or reclassified the star based on the available literature.

Abt \& Morrell (1995) gave the spectral type B9.5 Vp(Lam Boo)n, but we have reobserved the star and give the spectral type A0 IIIn. We think the rapid rotation gave the appearance of weakness in the 4481 line that Abt \& Morrell (1995) detected. Evidence for an expanding gas shell was presented by Hauck et al. (1998), which suggests the weak 4481 line may also have a shell origin, though we did not see this ourselves. Recommendation: non-member.

\subsection{HD 34797}

Proposed by Slettebak (1963) as an uncertain member of the $\lambda$ Boo group. Membership can be excluded on the grounds of its $\alpha^{2} \mathrm{CVn}$ nature, and having a spectral type 'B7 Vp He wk' (Abt \& Cardona 1983). Meets none of the UV criteria for membership in Faraggiana et al. (1990). Listed as misclassified in the Renson et al. (1990) catalogue. Recommendation: non-member.

\subsection{HD 35242}

Although already classified as A1 Vp(4481 weak) by Abt \& Morrell (1995), the $\lambda$ Boo nature of the object was first noted in the Paunzen et al. (2001) survey, for which Gray's notes on the spectrum read: 'This is clearly a Lambda Boo star. . . Hlines are an excellent match to Bet Leo [A3 Vas], but metals are clearly weaker, including $\lambda 4481$. Metals are closest to A0.5; $\mathrm{K}$ line is about A1'. The spectral type thus assigned was A3mA1 Va Lam Boo. Recommendation: member.

\subsection{HD 36496}

A mild $\lambda$ Boo star according to Abt (1984b), but Abt \& Morrell (1995) classified it as 'A5 Vn'. Its distance from the line of normal stars in the $\Delta a$ photometry study of Maitzen \& Pavlovski (1989b) is also consistent with mild $\lambda$ Boo peculiarity. No evidence for a shell in the IR (Andrillat et al. 1995). Faraggiana et al. (2004) listed the star as having a composite spectrum; in the WDS this object is one of a pair of stars separated by 0.2 arcsec with a magnitude difference of 1.0. uvby photometry is consistent with a star around A8 with metals around A1. A new spectral classification is needed. Recommendation: uncertain member.

\subsection{HD 36726}

A new $\lambda$ Boo star in the Paunzen \& Gray (1997) survey paper, where its spectrum is described as being very similar to HD 31295- one of the best-studied $\lambda$ Boo stars. They gave a spectral type of 'kA0hA5mA0 V $\lambda$ Boo'. The abundances show solar $\mathrm{C}$ and $\mathrm{O}$, but underabundances of refractory elements of between 0.5 and $1.0 \mathrm{dex}$ (Andrievsky et al. 2002). We reobserved this target and classified it as hA4 Vb kA0.5mA0.5 $\lambda$ Boo, noting the particularly broad $\mathrm{H}$ wings. Recommendation: member.

\subsection{HD 37411}

A Herbig Ae star, and dubbed a 'fully-fledged $\lambda$ Boo star' by Gray \& Corbally (1998), where it is given the spectral type ' $\mathrm{kA} 0 \mathrm{hA} 3 \mathrm{~mA} 0 \mathrm{Va}(\mathrm{e}) \lambda$ Boo' and is discussed at length. We also obtained a new spectrum for this work and give the classification hA2 Vae $\mathrm{kB} 8 \mathrm{mB} 8 \lambda$ Boo, noting clear emission in $\mathrm{H} \beta$. Recommendation: member.

\subsection{HD 37886}

This star was described in a paper on very young (1.7 Myr) HgMn stars (Woolf \& Lambert 1999), and it is this classification that leads us to reject the star. Recommendation: non-member.

\subsection{HD 38043}

Lies among the $\lambda$ Boo population in the $\Delta a$ photometry study of Maitzen \& Pavlovski (1989a), and is a $\lambda$ Boo star according to the Renson et al. (1990) catalogue. It is recorded in the first volume of the Michigan Catalogue (Houk \& Cowley 1975) as 'hF0kA4/7 metals very weak or invisible'. The discrepancy in the $b-y$ and $m_{1}$ indices (Eggen 1984) amounts to metal weakness of an entire spectral class. Paunzen et al. (2005) did not label the star as $\lambda$ Boo because it was classified earlier as a blue giant. We observed this star and give the spectral type F1 V kA5mA3 $\lambda$ Boo?. The 4481 line is very weak, but in early F stars this is blended with lines of Fe (see Section 1.4.1), and so an NLTE abundance analysis showing solar abundances of $\mathrm{C}, \mathrm{N}, \mathrm{O}$, and/or $\mathrm{S}$ is required to distinguish it from Pop. II stars. We do note that radial velocity and proper motions are very small, and thus tentatively accept it as a $\lambda$ Boo star for now. Recommendation: member.

\subsection{HD 38545 (HR 1989; 131 Tau)}

Identified as a $\lambda$ Boo star by Gray $\&$ Garrison (1987), with a classification 'A2 Va+ $\lambda$ Boo', and thus included as a $\lambda$ Boo star by Gray (1988). It does not lie far from the line of normal stars in the Maitzen \& Pavlovski (1989a) $\Delta a$ photometry study. An abundance analysis (Stürenburg 1993) shows very mild metal weakness; at around -0.5 dex, the abundances are only 1-2 $\sigma$ from solar for most refractories. Key 
physical parameters are available (Iliev \& Barzova 1995). Its $\lambda$ Boo nature has been discussed by Bohlender, Gonzalez, \& Matthews (1999), who reviewed the literature and suggested it may instead be an Ae or shell star, though we note that those classes do not ipso facto exclude membership in the $\lambda$ Boo group. Indeed, Bohlender \& Walker (1994) considered it a $\lambda$ Boo star with a shell. It was included in the Paunzen et al. (1997) consolidated catalogue of $\lambda$ Boo stars but was later dropped (Paunzen 2000) because of recently detected binarity. The WDS catalogue (Mason et al. 2001) lists a companion at 0.1 arcsec that is only 0.5 mag fainter. The star's rapid rotation (191 $\mathrm{km} \mathrm{s}^{-1}$, Royer, Zorec, \& Gómez 2007) may also have had some role in early identifications as a $\lambda$ Boo star, especially when combined with mild metal weakness. Our cursory inspection of an IUE UV spectrum shows that this star is normal, according to the UV criteria outlined by Faraggiana et al. (1990). The evidence argues against a $\lambda$ Boo classification. Recommendation: non-member.

\subsection{HD 39283}

One of the first $\lambda$ Boo stars recorded (Slettebak 1952), but it is actually a normal star-classified as 'A1 Va' (Gray \& Garrison 1987), it lies on the line of normal stars in the $\Delta a$ photometry study of Maitzen \& Pavlovski (1989b) and according to Faraggiana et al. (1990) the UV character of the star does not warrant inclusion in the $\lambda$ Boo group. They added that it might have 'some slight $\mathrm{Al}$ deficiency' and that it is a 'possible weak-line star'. It was thus given a misclassified ('/) designation in the Renson et al. (1990) catalogue. Strong evidence for a shell was presented by Andrillat et al. (1995), who also called the star $\lambda$ Boo but possibly only in reflection of the literature. Gerbaldi et al. (2003) wrote 'inconsistent UV flux (solar ab.)', thus suggesting rejection. Recommendation: non-member.

\subsection{HD 39421}

Falls among the $\lambda$ Boo population in the $\Delta a$ photometry study of Maitzen \& Pavlovski (1989a). Described as an A2 Vp star with strong O I and S I star in the IR study of Andrillat et al. (1995). Classified as A1 Va (wk 4481) by Paunzen et al. (2001), but not as a $\lambda$ Boo star, even though the star was included in the consolidated list of Paunzen et al. (1997). Abt \& Morrell (1995) also noted the $\lambda 4481$ weakness. $u v b y \beta$ photometry (Hauck \& Mermilliod 1998) indicates a hydrogen type about A4 from both $\beta$ and $b-y$, for which the $m_{1}$ index of 0.161 is only slightly small. UV spectrum analysis shows a $\lambda$ Boo nature (this work). However, our re-investigation of the star's optical spectrum yielded the spectral type A1 Vn, i.e. not $\lambda$ Boo. Earlier detections of a weak 4481 lines might be due to the rapid rotation. In light of the mixed evidence, we leave the membership as uncertain. Recommendation: uncertain member.

\subsection{HD 40588}

Abt \& Morrell (1995) noted a weak $\lambda 4481$ line in this star, and Paunzen et al. (2001) classified it as 'A1 Va (sl weak 4481)' but neither paper refers to the star as a $\lambda$ Boo star. Andrievsky et al. (2002) confirmed membership in the $\lambda$ Boo group with an LTE abundance analysis, showing approximately solar $\mathrm{C}$ and $\mathrm{O}$, but deficient $\mathrm{Mg}$ and -1 dex abundances of $\mathrm{Fe}$ and $\mathrm{Cr}$. We reobserved this target and give the spectral type A3 V kA0.5mA0 $\lambda$ Boo. Recommendation: member.

\subsection{HD 41580}

Classified 'A0 Vp lambda Boo' by Abt (1985), and appears in the Renson et al. (1990) catalogue. Not suggested for rejection by Gerbaldi et al. (2003). No real evidence in favour of inclusion into the class, and our new observations indicate the spectral type A1 IIIp Si. Recommendation: non-member.

\subsection{HD 42503}

An A2 $\mathrm{V} \lambda$ Boo star (Paunzen et al. 2002b). $v \sin i$ was not determined, but $\log g=3.1$ is very low for a $\lambda$ Boo star. [Z] was given as $-0.83 \pm 0.20$. Handler's (1999) $u v b y \beta$ photometry gives consistent hydrogen line strengths from $b-y$ and $\beta$ that suggest an $\sim \mathrm{A} 8$ dwarf, with an $m_{1}$ index about as small as an A0 star. We observed this star and give the spectral type hA9 Vn kA2mA2 $\lambda$ Boo. Recommendation: member.

\subsection{HD 47152}

The essential notes on SIMBAD for this object, 53 Aur, indicate it is a $\lambda$ Boo star. This is incorrect. It was classified as a $\lambda$ Boo star by Abt \& Morrell (1995) but later dismissed by Hauck et al. (1998) because of its 'well-known' identification as an $\mathrm{Hg}$ star (they referenced Osawa 1965). Zverko et al. (2008) revisited this system to give a definitive classification. They determined B9 Mn for the primary, and F0m for the secondary, i.e. neither object is a $\lambda$ Boo star. ${ }^{5}$ Recommendation: non-member.

\subsection{HD 54272}

Classified by Paunzen et al. (2001) as kA3hF2mA3 V LB and included in the results paper of that series with the comment 'very metal-weak star' (Paunzen 2001). No $\delta$ Sct pulsational variability at the 1.4-mmag level (Paunzen et al. 2002b). Gerbaldi et al. (2003) noted 'inconsistent UV flux (bin.?)'. On Gray's list of late $\lambda$ Boo candidates, though in his notes to Paunzen, Gray writes 'FBS?? (This is one of Olsen's FBS).' [FBS = field blue straggler.] Proper motions (0.8 and

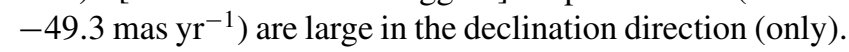

\footnotetext{
${ }^{5} \mathrm{~A}$ recommendation has been made that the SIMBAD administrators remove the note that states this star is a $\lambda$ Boo star.
} 
Previously classified as an RRab pulsator, with 0.1-mag light variation and 0.779-d pulsation period (Szczygieł \& Fabrycky 2007; Skarka 2013), but recently overturned in favour of $\gamma$ Dor pulsation by Paunzen et al. (2014a) who gave a new spectral type of $\mathrm{kA} 3 \mathrm{hF} 2 \mathrm{~mA} 3 \mathrm{~V} \mathrm{LB}$, and found $v \sin i=250 \pm 25 \mathrm{~km} \mathrm{~s}^{-1}$ and $[M / \mathrm{H}]=-0.8$ to $-1.1 \mathrm{dex}$. The quoted $v \sin i=250 \mathrm{~km} \mathrm{~s}^{-1}$ is very rapid for an $\mathrm{F}$ star. From three spectra taken with two different instruments, we find that HD 54272 is not a rapid rotator. We could not find any literature $v \sin i$ values against which to compare. Yet the spectrum that Paunzen et al. (2014a) presented does look broad lined. We tentatively suggest that the spectrum they gave is misidentified.

Our new spectra do show clear metal weakness, but there is no evidence that this is a $\lambda$ Boo star $(\lambda 4481$ is borderline normal, and is blended with Fe in F2 stars; see Section 1.4.1). We give the spectral type F1 V kA6mA3. Since this is not a rapid rotator, a high-resolution spectrum including lines of volatile elements could provide a definitive membership assessment. Recommendation: uncertain member.

\subsection{HD 56405}

This star was given an uncertain designation in the Renson et al. (1990) catalogue, presumably because it had only been identified as a $\lambda$ Boo candidate from Abt's (1984a) work. Gerbaldi et al. (2003) rejected the star because of 'inconsistent UV flux (bin.?)' and noted RV variability. Rejected earlier by Paunzen et al. (1997) because it was identified by Gray \& Garrison (1987) as a high $v \sin i$ A2 Va standard. Recommendation: non-member.

\subsection{HD 64491}

First classified as $\lambda$ Boo by Abt \& Morrell (1995), its $\lambda$ Boo nature was confirmed by Paunzen \& Gray (1997) who gave the spectral type 'kA3hF0mA3 V $\lambda$ Boo'. Kamp et al. (2001) derived $[\mathrm{N}]$ and $[\mathrm{S}]$ abundances of -0.30 and -0.09 , respectively, when considering the star as single. However, in a dedicated paper where this star's literature coverage is documented in detail, Faraggiana \& Gerbaldi (2003) presented evidence that HD 64491 is not a single object of $[M / \mathrm{H}]$ $\sim-1.5$ dex, but is better explained as two slightly metal poor objects $(\sim-0.5 \mathrm{dex})$ of similar temperature and different $v \sin i$. Their poor temporal coverage leaves their SB2 detection as a borderline one in significance, and depending on the exact metallicity of the two objects, they may have found that this object is composed of two mild $\lambda$ Boo stars rather than one single one. We obtained two spectra of this star and each has the spectral type F1 Vs kA3mA3 $\lambda$ Boo. Recommendation: member.

\subsection{HD 66684}

Close visual binary, primary is B9 Va and secondary A1 IVn (Gray \& Garrison 1987). Abt \& Morrell (1995) classified the
B component as A0 Vp (4481 wk)n and the A component as B9.5 Vp(Lam Boo). Paunzen et al. (2001) gave the classification as B9.5 Va (sl wk met) but did not state whether this was the A or B component. LTE abundance analysis revealed underabundance of 1 dex in Fe, but $\lambda$ Boo membership could not be ascertained (Andrievsky et al. 2002). Ironically, Gerbaldi et al. (2003) rejected HD 66684 because of 'discordant $v \sin i$ values', not because of binarity. Our own classification is based on one new spectrum when the seeing was not good enough to resolve the two components (the separation is $\sim 2.5 \mathrm{arcsec}$ ). We give the spectral type B9.5 Va (wk 4481), but we do not consider it a $\lambda$ Boo star. Recommendation: non-member.

\subsection{HD 66920}

This star is allegedly misclassified as a $\lambda$ Boo star (Paunzen et al. 2002b), since Paunzen et al. (2001) gave the nonpeculiar classification A3 V. $\Delta a$ photometry (Vogt et al. 1998) does not support a $\lambda$ Boo classification. Recommendation: non-member.

\subsection{HD 68695}

Spectral type 'A0 V' according to Houk (1978). Folsom et al. (2012) described this Ae star as having 'clear $\lambda$ Boo peculiarities', with $\mathrm{C}, \mathrm{N}, \mathrm{O}$, and $\mathrm{S}$ being $1 \sigma$ above solar, and Fe-peak elements being 2-3 $\sigma$ below solar. We obtained two spectra at different times for this work, from which we conclude the star is probably a spectrum variable; our best spectral type is A3 Ve kA1mA0, in which we note strong emission in $\mathrm{H} \beta$, but it does not appear to be a $\lambda$ Boo star generally. We leave the membership as uncertain, and suggest an investigation of the UV spectrum to shed further light. Recommendation: uncertain member.

\subsection{HD 68758}

Very rapid rotator $\left(\geq 270 \mathrm{~km} \mathrm{~s}^{-1}\right)$; only $[\mathrm{Fe} / \mathrm{H}]$ could be determined $(-0.58)$ by Solano et al. (2001), who also gave a mass of $2.4 \mathrm{M}_{\odot}$ and an age of $427 \mathrm{Myr}$. It was given the spectral type A1 IVp by Paunzen et al. (2001), but the exact peculiarity is not specified. If it were a $\lambda$ Boo star, that would have been indicated. Probably just a fast rotator. Recommendation: non-member.

\subsection{HD 73210}

Pavlovski, Schnell \& Maitzen (1993) used the $\Delta a$ photometry method to find three new $\lambda$ Boo stars in the Praesepe cluster: KW 50, KW 114, and KW 375, which are HD 73210, 73345, and 73872. Each of the three stars was refuted later by Gray \& Corbally (2002). HD 73210 was rejected because it was a A5 IIIs standard (Gray \& Garrison 1989b). The other two stars have spectral types A7 V kA8 and 
kA6hA8mA6 V(n), respectively (Gray \& Corbally 2002), i.e. none is a $\lambda$ Boo star. Recommendation: non-member.

\subsection{HD 73345}

See HD 73210. Recommendation: non-member.

\subsection{HD 73872}

See HD 73210. Recommendation: non-member.

\subsection{HD 74873}

Identified as a 4481-weak star by Abt \& Morrell (1995), and considered as a $\lambda$ Boo star by Kuschnig et al. (1996). Paunzen \& Gray (1997) included this as one of their new $\lambda$ Boo stars and gave it a more precise spectral type of ' $\mathrm{kA} 0.5 \mathrm{hA} 5 \mathrm{~mA} 0.5 \mathrm{~V} \lambda$ Boo'. There is disagreement in the literature over this star's $v \sin i$ value (see Heiter 2002 for a discussion). Heiter (2002) determined $v \sin i=130 \mathrm{~km} \mathrm{~s}^{-1}$ from high-resolution spectroscopy, and performed an abundance analysis showing moderate $(0.5 \mathrm{dex})$ underabundances of metals and overabundances of volatiles, i.e., HD 74873 has a $\lambda$ Boo abundance pattern. Paunzen et al. (2003) noticed an infrared excess that they attributed to dust. Gerbaldi et al. (2003) noted variable RVs had been reported for this star, though speckle interferometry had yielded no companion. They reported an inconsistent UV flux, in that solar abundances fit better than $\lambda$ Boo-like abundances in the UV, which Faraggiana et al. (2004) interpreted as evidence of a companion (despite one not being found). Recommendation: member.

\subsection{HD 74911}

Candidate $\lambda$ Boo star in Paunzen \& Gray (1997), where its spectral type was given as A2 IV (wk 4481). Classified as A3 IV by Paunzen et al. (2001), who were specifically looking for $\lambda$ Boo stars, but Andrievsky et al. (2002) 'confirmed' its $\lambda$ Boo membership with an abundance analysis showing metal deficiency ( -0.8 dex) alongside a $[\mathrm{C}]$ abundance of -0.25 dex. Strömgren photometry is consistent with a midA star with mild metal weakness. Our new spectrum yields the spectral type A8 Vn kA1mA1, from which we also conclude this star is just metal weak-it is not a $\lambda$ Boo star. Recommendation: non-member.

\subsection{HD 75654}

Serendipitously discovered to be variable by Balona (1977) with $11.5 \mathrm{~d}^{-1}$ variability, and later confirmed as a multiperiodic $\delta$ Sct star by Paunzen et al. (2002b). Hauck (1986) adopted HD 75654 as a $\lambda$ Boo candidate upon noticing it meets the photometric conditions for a $\lambda$ Boo star-a fact corroborated by Maitzen \& Pavlovski (1989a) who showed it to lie among the $\lambda$ Boo stars in a $\Delta a$ photometry. Included as a $\lambda$ Boo star in the Renson et al. (1990) catalogue. [C], [N], $[\mathrm{O}]$, and $[\mathrm{S}]$ abundances are $-0.44,+0.30,+0.14,-0.10$, respectively (Paunzen et al. 1999b; Kamp et al. 2001), which alongside abundances of metals of -1 dex in table 4 of Solano et al. (2001) support a $\lambda$ Boo classification. Included in Paunzen's (2001) results paper as a confirmed $\lambda$ Boo star with spectral type 'hF0mA5 V $\lambda$ Boo'. Mentioned as 'a probable double' by Faraggiana et al. (2004). A $\lambda$ Boo star on Gray's website with spectral type 'A7 kA3mA3 V $\lambda$ Boo'. Recommendation: member.

\subsection{HD 78316}

Misclassified as a $\lambda$ Boo star, according to Renson et al. (1990). It is a well-known Ap star, with spectral type 'B8IIIp HgMnEu MgII 4481A weak' (Abt \& Morrell 1995). The WDS catalogue (Mason et al. 2001) lists a companion at 0.2 arcsec that is $3.5 \mathrm{mag}$ fainter. Recommendation: non-member.

\subsection{HD 78661}

An uncertain member of the $\lambda$ Boo group (Renson et al. 1990), this star was rejected from the Paunzen et al. (1997) consolidated catalogue because it is an F2 star. IRAS infrared excess detected (King 1994). According to the WDS catalogue (Mason et al. 2001), this is a suspected occultation binary. The spectral type given by Abt \& Morrell (1995) is ' $\mathrm{F} 2 \mathrm{~V} \mathrm{mA8'}, \beta$ and $b-y$ are in rough agreement with each other, and support the F2 type, but $m_{1}$ indicates a metal type about four spectral subclasses earlier. This is in agreement with the metallicity of $[\mathrm{Fe} / \mathrm{H}]=-0.55$ in Nordström et al. (2004), and with our classification of a new spectrum, which is F2 V kA7mA7. We noted that the spectrum is metal weak, but $\lambda 4481$ is not unusually weak. Recommendation: non-member

\subsection{HD 79025}

Classified as A9 Vn by Paunzen et al. (2001), who were specifically looking for $\lambda$ Boo stars. Among the normal (or even enhanced metals) stars in a $(b-y, \Delta a)$ diagram, based on values catalogued by Vogt et al. (1998). Recommendation: non-member.

\subsection{HD 79108}

Not far from the line of normal stars in the Maitzen \& Pavlovski (1989a) $\Delta a$ photometry study. Included in the Renson et al. (1990) catalogue, and classified as A0 V $\lambda$ Boo by Abt \& Morrell (1995). An RV variable according to Gerbaldi et al. (2003), and listed as a star with a composite spectrum by Faraggiana et al. (2004), where in their section 3.2 their conclusion is that this star is 'in reality a complex system composed of at least two stars of similar luminosity and a third less luminous component'. In any case, the star is only 
slightly metal weak $([\mathrm{Fe} / \mathrm{H}]=-0.07$, Saffe et al. 2008) and some doubt has arisen over the $v \sin i$ value, in that those authors tabulate only $10.2 \mathrm{~km} \mathrm{~s}^{-1}$ and their reference does not follow up. Recommendation: non-member.

\subsection{HD 79469}

One of the first $\lambda$ Boo stars recorded (Morgan, Keenan \& Kellman 1943), but classified as 'B9.5 IV (CII)' by Gray $\&$ Garrison (1987). Falls exactly on the line of normal stars in the Maitzen \& Pavlovski (1989a) $\Delta a$ photometry study. Faraggiana et al. (1990) concluded this is not a $\lambda$ Boo star because it meets none of the UV requirements for membership. It is 'misclassified' according to the Renson et al. (1990) catalogue. Recommendation: non-member.

\subsection{HD 80081}

A lack of $\lambda$ Boo character in the UV led Paunzen et al. (1997) to declassify this star from a $\lambda$ Boo member, in accordance with the papers on the UV spectra of $\lambda$ Boo stars by Baschek et al. (1984) and Faraggiana et al. (1990). Lies directly on the line of normal stars in the Maitzen \& Pavlovski (1989b) $\Delta a$ photometry study. Classified as 'A1 V' by Abt \& Morrell (1995) and as A2 $\mathrm{IV}^{-}$by Gray \& Garrison (1987). There are indications of multiplicity in the WDS catalogue (Mason et al. 2001). Recommendation: non-member.

\subsection{HD 81104}

Abt (1985) gave the spectral type A2 Vn $\lambda$ Boo, but Bidelman, Ratcliff, \& Svolopoulos (1988) did not note peculiarity in their spectral type of A3 Vn and Paunzen et al. (2001) recorded a spectral type A3 Van, i.e. not $\lambda$ Boo although they were specifically looking for $\lambda$ Boo stars. Rejected by the Paunzen et al. (1997) catalogue, for inferences made in that survey. Recommendation: non-member.

\subsection{HD 81290}

Among the $\lambda$ Boo stars in in the Maitzen \& Pavlovski (1989a) $\triangle a$ photometry study. Classified as ' $\mathrm{kA} 5 \mathrm{hF} 3 \mathrm{mA5} \mathrm{V}$ LB' by Paunzen et al. (2001). Metal deficiencies are at about -1 dex (Solano et al. 2001). From our new spectrum, we give the spectral type hF2 $\mathrm{V} \mathrm{kA3mA3}$ ( $\lambda$ Boo?), meaning that it is a candidate as a mild $\lambda$ Boo star, but the late $\mathrm{H}$ line type is concerning (see Section 1.4.1). An NLTE analysis of the volatile elements is necessary to eliminate the possibility that this is a FHB star. Recommendation: probable member.

\subsection{HD 82573}

Claimed to be misclassified by Paunzen et al. (2002b) because it had been classified by Paunzen et al. (2001) as a normal star (A3 V). It is also normal according to Abt \& Morrell (1995, A7 V). uvby $\beta$ photometry (Hauck \& Mer- milliod 1998) indicates a slightly high $c_{1}$ index, while the $m_{1}$ index is what one expects given the $\beta$ and $b-y$ indices. Recommendation: non-member.

\subsection{HD 83041}

Very far from the line of normal stars in the Maitzen \& Pavlovski (1989a) $\Delta a$ photometry study, and a late but distinct $\lambda$ Boo member according to their figure 1. Spectral type of kA2hF2mA2 V LB (Paunzen et al. 2001). Metal weakness of over 1 dex (Solano et al. 2001). Rejected by Gerbaldi et al. (2003) because it was considered to be a FHB star with $\lambda$ Boo properties by Corbally \& Gray (1996), who actually classified it as 'F2 V kA3mA2 lam Boo PHL'. The measured radial velocity there was $46 \mathrm{~km} \mathrm{~s}^{-1}$. Gray, Corbally, \& Philip (1996) claimed this star lies significantly below the zero-age horizontal branch (ZAHB) and suggested it is a field blue straggler. We reobserved this star and give the spectral type $\mathrm{F} 1 \mathrm{~V} \mathrm{kA} 3 \mathrm{~mA} 2 \lambda$ Boo, but note that due to the late hydrogen line type, an NLTE abundance analysis should be carried out to ensure a $\lambda$ Boo abundance pattern is shown, and that this is not just a metal weak or horizontal branch star. Recommendation: probable member.

\subsection{HD 83277}

Paunzen et al. (2001) gave the spectral type kA3hF2mA3V LB. Gerbaldi et al. (2003) noted an inconsistent UV flux and possible binarity. We observed this star for this work and give the spectral type F1.5 V kA3mA3 $\lambda$ Boo?, indicating it is either a late $\lambda$ Boo star or Pop. II. A full abundance analysis including volatile elements is required. Recommendation: probable member.

\subsection{HD 84123}

A abundance analysis (Heiter et al. 1998) confirms this star is a member of the $\lambda$ Boo group with $[Z]=-1.0$. $T_{\text {eff }}=6800 \mathrm{~K}$ and $\log g=3.5$. It was given the spectral type 'kA6hF1mA6 V (LB)' by Paunzen et al. (2001). Gerbaldi et al. (2003) argued misclassification because of high space velocities, but the abundance pattern (Heiter et al. 1998) is truly $\lambda$ Boo, with $[\mathrm{C}],[\mathrm{N}],[\mathrm{O}]=-0.1,0.0$ and $-0.3 \mathrm{dex}$, respectively. The radial velocity is only $16.3 \mathrm{~km} \mathrm{~s}^{-1}$ and the

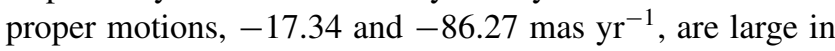
the declination direction (only). Our new spectrum yields the spectral type hF2 V kA6mA6 $\lambda$ Boo. Recommendation: member.

\subsection{HD 84948}

Among the $\lambda$ Boo population in the Maitzen \& Pavlovski (1989b) $\Delta a$ photometry study. Andrillat et al. (1995) noted 'LB, shell' in their infrared study, where in their figure 9 they show the asymmetric Paschen lines and redshifted $\mathrm{Ca}$ and $\mathrm{O}$ lines, but this is probably due to the binary 
nature. Heiter (2002) performed an abundance analysis, finding $\sim 1$ dex metal underabundances for the A component, and slightly smaller underabundances for the B component. The atmospheric parameters used were those of Paunzen et al. (1998a) which were challenged by Faraggiana et al. (2001b), which might explain the discrepant abundances for the two members, but which might also point to a triple system. Paunzen et al. (2001) gave the spectral type 'kA7hF1mA6 V (LB)', and from our new spectrum we give F1.5 Vs kA5mA5 $\lambda$ Boo?. NLTE abundances of the volatile elements would help arbitrate between a true $\lambda$ Boo and a Pop. II star. Recommendation: probable member.

\subsection{HD 87271}

Handler (1999) took $u v b y \beta$ photometry for HD 87271. $\beta$ (= $2.775)$ and $b-y(=0.151)$ are in agreement and correspond to a star with a hydrogen line type around A9, but the $m_{1}$ index is remarkably low, even lower than that expected of a B8 dwarf. Handler, Gray, \& Shobbrook (2000) followed up on the object with classification-resolution spectroscopy and described it as one of the most extreme classical $\lambda$ Boo stars known. They derived the spectral type A9 kA0mA0 V $\lambda$ Boo; we arrived at the same spectral type from our new spectrum. Recommendation: member.

\subsection{HD 87696}

Abt \& Morrell (1995) gave the spectral type A9 Vp (Boo; met: A5). According to Griffin et al. (2012), this star 'appears to be a single, rotationally broadened late-A star and has been widely classified as A7 V, with near-solar metallicity'. Recommendation: non-member.

\subsection{HD 89239}

Given the spectral type of A2 Vp(Boo; met:B9.5) in the Abt \& Morrell (1995) catalogue. No other evidence in favour. The $u v b y \beta$ data on SIMBAD show discordant Strömgren indices, but our new spectrum suggests this is a normal B9.5 V star.Recommendation: non-member.

\subsection{HD 89353}

Post-AGB star (Kohoutek 2001), discussed here because it was in the Renson et al. (1990) catalogue as an uncertain member. $u v b y \beta$ photometry (Hauck \& Mermilliod 1998) clearly confirms this is not an A-type dwarf. Recommendation: non-member.

\subsection{HD 90821}

Presented as a new $\lambda$ Boo star with spectral type 'kA2hA7mA2 Vn $\lambda$ Boo' and a comment 'normal hydrogen lines' by Paunzen \& Gray (1997), and included in the results paper of that series (Paunzen 2001). Andrievsky et al.
(2002) performed an LTE abundance analysis and wrote that HD 90821 'can be definitely ruled out as being [a member] of the $\lambda$ Bootis group', adding 'the abundances derived for seven elements do not show any significant deviation from solar values'. The solar metallicity is corroborated by a $[\mathrm{Fe} / \mathrm{H}]$ value of -0.03 in the PASTEL catalogue (Soubiran et al. 2010). We have obtained a new spectrum and classify HD 90821 as a normal A3 IV-V star. Recommendation: non-member.

\subsection{HD 91130}

HD 91130A = HR 4124. Abt \& Morrell (1995) classified this star as 'A0 Vp(Lam Boo)', which was confirmed by Paunzen \& Gray (1997) with the spectral type 'A0 Va- $\lambda$ Boo (PHL)' and a comment that its hydrogen lines are shallow and broad. Gray has reclassified that spectrum for this work, giving the spectral type 'kA0hA1mA0 Va ( $\lambda$ Boo)', and he noted a very weak $\lambda 4481$ line compared to the A0 standard. NLTE abundances for $[\mathrm{N}],[\mathrm{O}]$, and $[\mathrm{S}]$ are typical for $\lambda$ Boo stars at $-0.30,0.14$, and 0.18 , respectively (Paunzen et al. 1999b; Kamp et al. 2001). LTE abundances of refractory elements from Andrievsky et al. (2002) are around -1 dex, with -1.7 dex for [Fe]. Recommendation: member.

\subsection{HD 97411}

Abt \& Morrell (1995) described HD 97411 (HR 4347) as A0 IVp (4481-wk) with $v \sin i=25 \mathrm{~km} \mathrm{~s}^{-1}$. Paunzen et al. (1997) rejected HD 97411 based on Gray's (1988) comments. Gray wrote: 'The 4481 line is present and appears to be of quite normal strength. Hence, there appears to be no compelling reason to include this star in the $\lambda$ Boo class'. He added that any peculiarities could be produced by the close binary nature (sep. $0.2^{\prime}, \Delta V=0.4 \mathrm{mag}$ ) of this star. Recommendation: non-member.

\subsection{HD 97773}

Intermediate between the $\lambda$ Boo stars and normal stars in the Maitzen \& Pavlovski (1989b) $\Delta a$ photometry study. Membership is uncertain according to the Renson et al. (1990) catalogue. In their infrared study, Andrillat et al. (1995) listed a spectral type of A8 Vwl, with a metallic line type of A3, and 'LB?', and no indication of a shell. Gerbaldi et al. (2003) wrote 'composite, Hipparcos' next to this star, and it was therefore recorded by Faraggiana et al. (2004) as a $\lambda$ Boo star with a composite spectrum. The WDS catalogue (Mason et al. 2001) lists a companion at 0.3 arcsec of similar magnitude. We obtained a new spectrum that did not resolve the binarity and that we found difficult to classify. We give the type F1 Vn kA7mA5, with the comment: 'a very rapid rotator, which causes some uncertainty in the metal line type, even when comparing to A5 Vn and A7 Vn standards. The Mg II 4481 line looks weak even compared to the rapidly rotating standards, but an artificially broadened spectrum of the 
F0 Vs standard, HD 23585, up to $v \sin i=250 \mathrm{~km} \mathrm{~s}^{-1}$ has approximately the same $\mathrm{Mg}$ II 4481 strength'. Its $\lambda$ Boo nature remains uncertain. Recommendation: uncertain member.

\subsection{HD 97937}

Lies among the $\lambda$ Boo stars in the Maitzen \& Pavlovski (1989a) $\Delta a$ photometry study, but was given an uncertain membership likelihood in the Renson et al. (1990) catalogue. Classified as A9 Vp 4481-wk (Abt \& Morrell 1995). Rejected by Paunzen et al. (1997) because it was allegedly classified by Gray (1989) as an evolved star, but inspection of Gray's work shows a metallicity class -1.5 , but a spectral type of F0 V, i.e. not evolved. No abundances, but $u v b y \beta$ photometry (Hauck \& Mermilliod 1998) shows the $\beta$ and $b-y$ indices are in agreement with an early $\mathrm{F}$ star and the $c_{1}$ index is consistent with a luminosity class $\mathrm{V}$ object there. Then, the $m_{1}$ index suggests metal weakness by about 10 spectral subclasses. We obtained a new spectrum and give it the spectral type F1 V kA9mA6, but we note that the discrepancy between the $\mathrm{K}$ line and metallic-line types suggest this is a composite spectrum. We assign the star to the 'uncertain' membership category, pending a full abundance analysis. Recommendation: uncertain member.

\subsection{HD 98353 (55 UMa)}

Abt (1984a) noted the peculiarity of this object, that was later accepted as a $\lambda$ Boo star in the Renson et al. (1990) catalogue. Earlier investigations into its UV properties revealed no peculiarities; a spectral type of A2 was derived (Cucchiaro et al. 1980). Andrillat et al. (1995) presented substantial evidence for a shell. Long known to be a spectroscopic binary system, characterised by Batten, Fletcher, \& Mann (1978) as having an orbital period of $2.5 \mathrm{~d}$. Its binarity has been the subject of dedicated papers; Lloyd (1981) confirmed the orbital period and noted a high eccentricity $(e=0.43)$, which is very odd given the short orbital period - Lloyd calculated that the orbit should have circularised within 5 Myr. Lloyd concluded that at least one component of the system appears to have a UV excess, consistent with a $\lambda$ Boo star, but given the estimated mass ratio of the objects of 0.9 , it was not possible to say which. Gray \& Garrison (1987) classified this star as A1 Va with the comment 'Cores of the hydrogen lines look slightly washed out. A composite spectrum?' In a later works, Horn et al. (1996, and references therein) developed the picture of a triple system for $55 \mathrm{UMa}$, where the third component has a 1870-d orbital period. With synthetic spectra they obtained a best fit of $T_{\text {eff }}=9500 \mathrm{~K}$ and $\log g=4.5$ for both the primary and tertiary components, with $v \sin i=33$ and $55 \mathrm{~km} \mathrm{~s}^{-1}$, respectively, but the secondary could only be determined to be of spectral type A. In a tomographic separation of the constituent spectra, Liu et al. (1997) refined the $T_{\text {eff }}$ values of each member to $9230 \pm 230,8810 \pm 250$, and $9290 \pm 190$ for components $\mathrm{Aa}, \mathrm{Ab}$, and B respectively.
Their synthetic spectrum analysis showed that all the components, but particularly Aa and Ab of the close binary, had enhanced metal contents, and were consistent with the class of marginal Am stars. This directly conflicts with a $\lambda$ Boo classification. Recommendation: non-member.

\subsection{HD 98772}

Included in the Renson et al. (1990) catalogue, but determined not to be a $\lambda$ Boo star by Andrievsky et al. (2002) because it had only been described as a rapidly rotating normal or 4481-weak star in the past, and they found the abundances were not typical for a $\lambda$ Boo star - the abundances of $\mathrm{Mg}$ and $\mathrm{Si}$ are solar. In fact, it was in the consolidated catalogue of $\lambda$ Boo stars (Paunzen et al. 1997) but not the following paper in the series (Paunzen et al. 2001), where its spectral type was A1 Va. Recommendation: non-member.

\subsection{HD 100546}

'A clear $\lambda$ Boo star' (Acke \& Waelkens 2004). Also one of the closest and brightest HAeBe objects, and a $\beta$ Pic-like object. Classified as 'B9 Vne' by Houk \& Cowley (1975), and our new spectrum has the type $\mathrm{A} 0$ Vae $\mathrm{kB} 8$, with clear emission in $\mathrm{H} \beta$ and almost absent metals. It does have a $\lambda$ Boo character, in that the $\lambda 4481$ line is weak, but due to the near-absence of metal lines in the classification spectrum with which to compare the $\lambda 4481$ weakness, we cannot rule out that the star is just metal-weak overall, rather than a $\lambda$ Boo star. We have also inspected the available $I U E$ spectrum, which looks normal. Specifically, there is no $1600-\AA$ flux depression. Mulders et al. (2013) ruled on the sub-stellar companion associated with this star, concluding it is a $60_{-40^{-}}^{+20}$ $\mathrm{M}_{\text {Jup }}$ brown dwarf. Recommendation: uncertain member.

\subsection{HD 100740}

Classified in Paunzen et al. (2001) as kA4hA8mA5 V, but not as $\lambda$ Boo even though mild metal weakness is apparent. It is not classified as a $\lambda$ Boo anywhere else, and our new spectrum shows that the star has a normal $\lambda 4481$ line. It is slightly metal weak though, even after accounting for the very rapid rotation. We give the spectral type A3 IVnn kA2.5mA2.5. Recommendation: non-member.

\subsection{HD 101108}

One of Hauck \& Slettebak's (1983) nine official $\lambda$ Boo stars. Falls among the $\lambda$ Boo population in the $\Delta a$ photometry study of Maitzen \& Pavlovski (1989b). Matches all of the UV criteria for membership in Faraggiana et al. (1990); our re-investigation of an $I U E$ spectrum agrees. Iliev \& Barzova (1993b) confirmed the PHL profile with a 8000-K core and an 8400-K wing. They also said of the spectrum that the Ca II $\mathrm{K}$ line was strong but metallic lines were otherwise weak. No shell recorded by Andrillat et al. (1995). Paunzen et al. (2001) 
did not classify it as a $\lambda$ Boo star, though, rather as A3 IV (wk 4481). Strangely, it was then adopted in the results paper (Paunzen 2001) as 'A3 IV-V ( $\lambda$ Boo)', but without a special note other than to say it was discussed in their outline paper (Paunzen \& Gray 1997), where the comment given was that it could not be classified as a $\lambda$ Boo star at first sight. Heiter (2002) later found a mild $\lambda$ Boo abundance pattern. The hydrogen lines in our spectrum match the A3 IV standards better than the $\mathrm{A} 7 \mathrm{~V}$ ones, and then the metal lines are not generally peculiarly weak. The $\lambda 4481$ line is still weak in that case, though. We give the spectral type A3 IV (4481wk), and conclude that this may be an extremely mild $\lambda$ Boo star. Recommendation: probable member.

\subsection{HD 101412}

Cowley et al. (2010) studied HD 101412 and noted it to be somewhere between a $\lambda$ Boo star and a Vega-like object. The $\lambda$ Boo characteristics were confirmed by Folsom et al. (2012), who also summarised literature magnetic field measurements. This star is unusual in that it is a magnetic $\lambda$ Boo star, with a longitudinal field of $500 \pm 100 \mathrm{G}$. It shows depletions in the intermediate volatile zinc, which has bearing on the gas-grain separation discussed in $\lambda$ Boo formation theory. We have classified a new spectrum of HD 101412 as A3 Va(e) kA0mA0 $\lambda$ Boo, noting an emission notch in $\mathrm{H} \beta$ and a weak $\lambda 4481$ line. Recommendation: member.

\subsection{HD 102541}

Strömgren colours suggest HD 102541 is a metal-weak dwarf (Gray \& Olsen 1991), contrary to the SIMBAD spectral type (A3 II/III, Houk 1982). Kuschnig, Paunzen, \& Weiss (1997) took a spectrum and derived a spectral type kA3hA5mA3 V (LB), noting the Mg II 4481 line is normal for A3 and not remarkably weak. Confirmed as a $\lambda$ Boo star by Paunzen (2001) with a spectral type. 'kA5hF0mA5 V LB', and in this work we give the spectral type A9 V kA4mA4 ( $\lambda$ Boo), with the parentheses denoting its mild $\lambda$ Boo character. A note in Gerbaldi et al. (2003) reads 'inconsistent UV flux (bin.?)' but no firm evidence to suggest binarity exists. This star does have an unusually large transverse velocity, which cannot be explained by large uncertainties in the parallax or proper motion measurements. A full abundance analysis should be carried out to be sure the abundance pattern matches that of the $\lambda$ Boo stars. Recommendation: probable member.

\subsection{HD 103483}

The spectral type, kA2hA5mA3 V, given by Paunzen et al. (2001) is consistent with a mild $\lambda$ Boo star, but they did not identify it as such. Andrievsky et al. (2002) performed an abundance analysis for this star, showing metal underabundances of $\sim 0.6 \mathrm{dex}$, but since oxygen was equally underabundant, they rejected it from the $\lambda$ Boo group. Also a detached eclipsing binary of the Algol type. In fact, it is part of a sextuple system (Zasche et al. 2012). Recommendation: non-member.

\subsection{HD 105058}

Its $\lambda$ Boo status was established by Slettebak, Wright $\&$ Graham (1968), who noted that the ratio of the Ca II K line with the hydrogen lines corresponded to a spectral type near A2, but Mg II 4481 and other metallic lines were weak for this type. Baschek et al. (1984) investigated the UV spectrum for this star, which has a moderate $\lambda$ Boo character, as confirmed by Faraggiana et al. (1990). Falls firmly in the $\lambda$ Boo group in the Maitzen \& Pavlovski (1989b) $\Delta a$ photometry study. Adopted as a definite member of the $\lambda$ Boo group in the Renson et al. (1990) catalogue. Its hydrogen lines are of the PHL type (Iliev \& Barzova 1993b), and show 'emissionlike' features (Iliev \& Barzova 1998). Andrillat et al. (1995) had nothing peculiar to report in the infrared. Paunzen \& Gray (1997) gave a spectral type 'kA1hA7mA1 V $\lambda$ Boo'. Andrievsky et al. (2002) found -1 dex metal underabundances in an LTE analysis, but concluded its $\lambda$ Boo nature is ambiguous because no abundances for $\mathrm{C}, \mathrm{N}, \mathrm{O}$, or $\mathrm{S}$ are available. From our spectrum, we obtain the spectral type hA8 V kA0.5mA0.5 $\lambda$ Boo. Recommendation: member.

\subsection{HD 105199}

Paunzen \& Gray (1997) discussed this star. With a spectral type there of 'kA0.5hF0mA3 V $\lambda$ Boo:' they wrote ' $[\mathrm{t}] \mathrm{his}$ would be the first $\lambda$ Boo star where the $\mathrm{K}$ line type is in gross disagreement with the metallic-line spectrum'. In his 'Miscellaneous spectroscopic notes', Bidelman (1988) entered the note 'early Am' for this star, in direct contravention of the expected metal weakness of $\lambda$ Boo stars. In this work, a 3.6- $\AA$ spectrum of this star was obtained that shows a clear, mild Am character with the spectral type kA2hA5mA5 (IV), with mildly enhanced Sr II 4077 (typical of Am stars), and a mild anomalous luminosity effect. The only deviation from an Am star is a slightly weak Mg II 4481 line. Definitely not a $\lambda$ Boo star. Recommendation: non-member.

\subsection{HD 105260}

An uncertain member of the class (Renson et al. 1990), and classified by Abt (1984b) as F0 V wl(met:A5). A reinvestigation of the spectrum is required. Recommendation: uncertain member.

\subsection{HD 105759}

Despite being sixth magnitude, HD 105759 was not discovered to be variable until 1991, with a 0.045-d period (Colomba, de Benedetto, \& Ielo 1991). Further frequencies were found and Strömgren photometry obtained by Koen et al. (1995); Strömgren photometry indicates a metal-poor star near A9. It was described as a multiperiodic $\delta$ Sct star 
with $[Z]=-1$ dex in a dedicated paper (Martinez et al. 1998), though no abundances for C, N, O, or S were determined. We refer the reader there for more information, including their justification for acceptance of this star into the $\lambda$ Boo group. We accept this star as a member based on their recommendation, but a spectrum is desired. Recommendation: member.

\subsection{HD 106223}

Slettebak (1968) described this object as having Balmer lines near F0, but 'all of the metallic lines, including the Ca II K line, are exceedingly weak for this spectral type, which is reflected in the very low $m_{1}$ index'. One of the stars furthest from the line of normal stars in the $\Delta a$ photometry study of Maitzen \& Pavlovski (1989b), and the reddest of the stars they studied. It was described as a cool $\lambda$ Boo star by Faraggiana et al. (1990), but was too cool for their UV membership criteria to be applied. It was accepted into the Renson et al. (1990) catalogue, and Iliev \& Barzova (1993b) confirmed a peculiar hydrogen line profile, whose cores matched a 6900$\mathrm{K}$ spectrum but whose wings matched a $7300-\mathrm{K}$ spectrum. They argued the hydrogen lines did not agree with suggestions that this is an FHB star. No evidence for a shell in IR observations was found by Andrillat et al. (1995). Observed to be constant at the 3-mmag level (Paunzen et al. 1998c). Given the spectral type 'kA3hF3mA3 V LB' by Paunzen et al. (2001), where an FHB nature was again ruled out. An abundance analysis confirmed $\sim 2$ dex underabundances of metals (Andrievsky et al. 2002). A spectrum obtained for this work shows a $\lambda$ Boo character, with spectral type F4 V kA1.5mA1 $\lambda$ Boo. We noted a weak G band and peculiar $\mathrm{H}$ lines. Recommendation: member.

\subsection{HD 107223}

Examined by Paunzen et al. (2002b) for pulsation, but was found to be constant at the 1.9-mmag level. That is the only literature reference for this star on SIMBAD, so we are forced to consider it may have been a typo of the confirmed member HD 107233 (see the next subsection) or HD 106223 (above). Strömgren photometry (Hauck \& Mermilliod 1998) shows $\beta=2.9$, which is as high as values of $\beta$ reach. However, the $m_{1}$ index $(=0.11)$ is discordant with that and would imply a metal weakness. This is another potential explanation for its appearance in the Paunzen et al. (2002b) paper. There is no evidence to suggest membership, and our spectrum shows this star to be an excellent match to the A1 IVs standard, $\rho$ Peg. Recommendation: non-member.

\subsection{HD 107233}

Gray \& Olsen (1991) took Strömgren photometry for this star in their work on supergiants-HD 107233 is classified as such on SIMBAD. The measurements were $b-y=0.192$, $m_{1}=0.110, c_{1}=0.710$, and $\beta=2.743$. Collectively, $b-y$, $c_{1}$ and $\beta$ are consistent with a dwarf near $\mathrm{F} 1$, but for such a star the $m_{1}$ index is exceedingly weak (though no note to that effect is provided ibid.). The next time HD 107233 appears in the literature is in a table of confirmed $\lambda$ Boo stars (table 2 of Gray \& Corbally 1993), with a spectral type kA1hF0mA1 Va $\lambda$ Boo, which was later repeated by Paunzen et al. (2001). Key physical parameters are available from Iliev $\&$ Barzova (1995), who found this star to be both the lowest mass $\left(1.6 \mathrm{M}_{\odot}\right)$ and the oldest (1 $\left.090 \mathrm{Myr}\right) \lambda$ Boo star then known. Solano et al. (2001) found a temperature of $6950 \mathrm{~K}$ and $v \sin i=110 \mathrm{~km} \mathrm{~s}^{-1}$. Heiter (2002) preferred $6800 \mathrm{~K}$ and $v \sin i=80 \mathrm{~km} \mathrm{~s}^{-1}$, and confirmed $\lambda$ Boo abundances with only slightly deficient $[\mathrm{C}](-0.3 \mathrm{dex})$ alongside average metal deficiencies of -1.4 dex. If the typo of HD 107223 (see the previous subsection) was meant to be 107233 in the Paunzen et al. (2002b) paper, then we conclude non-variability at the 1.9-mmag level. Recommendation: member.

\subsection{HD 108283}

A $\lambda$ Boo star according to Abt \& Morrell (1995), but Hauck et al. (1998) rejected it because its $\Delta m 2$ index is positive, with the same value as that of an Am or Ap star, and it has been classified as an HgMn star. Indeed, Gray \& Garrison (1989a) classified it as A9 IVnp Sr II. Recommendation: non-member.

\subsection{HD 108714}

Listed as an uncertain member of the $\lambda$ Boo group in the Renson et al. (1990) catalogue. Falls among the population of normal stars in the extended $\Delta a$ photometry study of Maitzen \& Pavlovski (1989a). $u v b y \beta$ photometry shows no discordance between the $m_{1}$ index and the $\beta$ and $b-y$ indices. Recommendation: non-member.

\subsection{HD 108765}

Paunzen et al. (2001) gave a three-part spectral type in their observational paper of ' $\mathrm{kA} 3 \mathrm{hA} 3 \mathrm{~mA} 0 \mathrm{~V}$ ', while Andrievsky et al. (2002) stated this star can definitely be ruled out as being a member of the $\lambda$ Boo group because the abundances were, within the errors, solar. Recommendation: non-member.

\subsection{HD 109738}

A comment on this star in Vol. I of the Michigan Catalogue (Houk \& Cowley 1975) describes the Ca II K line and metal lines as very weak. Consequently, HD 109738 appeared in Hauck's (1986) list of $\lambda$ Boo candidates. It appears well amongst the $\lambda$ Boo stars in the extended $\Delta a$ photometry study of Maitzen \& Pavlovski (1989a). A member of the $\lambda$ Boo group in the Renson et al. (1990) catalogue. Given a spectral type of 'kA1hA9mA1 V LB' in Paunzen's (2001) 'new and confirmed' list of $\lambda$ Boo stars. (As an aside, contrary to Paunzen in that 2001 article, this should have been 
a 'confirmed' rather than a 'new' $\lambda$ Boo star). A new spectrum obtained for this work confirms the $\lambda$ Boo membership with the spectral type A9 Vn kB9.5mA0 $\lambda$ Boo. Solano et al. (2001) derived metal underabundances of -1 dex, but no abundances for volatile elements are available. Recommendation: member.

\subsection{HD 109980}

Abt \& Morrell (1995) classified this star as A6 Vp(Lam Boo) with an uncertain $v \sin i=255 \mathrm{~km} \mathrm{~s}^{-1}$. The rapid rotation may be responsible for the perceived metal weakness. Spectral type in Gray, Napier, \& Winkler (2001) is 'A8 Vnn kA6', i.e. it was not identified them as a $\lambda$ Boo star. Recommendation: non-member.

\subsection{HD 110377}

Classified as A6 Vp(Lam Boo) by Abt \& Morrell (1995), but with $v \sin i=160 \mathrm{~km} \mathrm{~s}^{-1}$. Subject of a dedicated paper into its $\delta$ Sct variability (Bartolini et al. 1980), where the multiperiodicity was interpreted as radial pulsation. Well covered in the $\delta$ Sct literature. $u v b y \beta$ photometry (Hauck \& Mermilliod 1998) suggests a normal star around A5. No abundances to suggest membership. Recommendation: non-member.

\subsection{HD $110411(\rho$ Vir)}

Gray (1988) classified HD 110411 as A0 Va ( $\lambda$ Boo) with the comment ' $\lambda 4481$ very weak compared to the metallicline spectrum. Hydrogen line profiles are nearly normal, except wings appear shallow. This star is a variable with an amplitude of $0.02 \mathrm{~V}$. It is also a spectroscopic binary'. He has reclassified the star for this work, with the spectral type 'kA0hA3mA0 Va $\lambda$ Boo', and noted the $\lambda 4481$ line is weak compared to the A0 standard. HD 110411 falls far from the line of normal stars in the Maitzen \& Pavlovski (1989a) $\Delta a$ photometry study. Meets all the UV criteria for $\lambda$ Boo membership (Faraggiana et al. 1990). A $\lambda$ Boo member in the Renson et al. (1990) catalogue. LTE metal abundances for measured elements and ions sit at -1 dex (Stürenburg 1993). Key physical parameters are available from Iliev \& Barzova (1995), but their suggested age of $200 \mathrm{Myr}$ is inconsistent with the $500 \pm 200$ Myr age recorded by Vican (2012). Described as a dusty system by Cheng et al. (1992), but Holweger \& Rentzsch-Holm (1995) found no evidence of circumstellar dust in the $\mathrm{Ca}$ II $\mathrm{K}$ line, and concluded that any circumstellar component present at the time of observation must have been weaker than $3 \mathrm{~m} \AA$. However, substantial evidence for a debris disc does exist (Booth et al. 2013). Although HD 110411 appears in the online data of the second paper (Paunzen et al. 2001) of the series of papers by Paunzen, which searched for new $\lambda$ Boo stars, it is curiously not included in the 'new and confirmed' list of $\lambda$ Boo stars in the third (Paunzen 2001). The reason is that known $\lambda$ Boo stars were observed as comparison stars for the project; these stars were not included in print, but did appear in the online data of the second paper in the series (Paunzen, private communication). Heiter (2002) confirmed a $\lambda$ Boo abundance pattern, with both [C] and [O] being supersolar, though it was noted that strangely $\mathrm{Mg}$ and $\mathrm{Si}$ were not as underabundant as other metals. Although some evidence is conflicting, there is enough to support $\lambda$ Boo membership. Recommendation: member.

\subsection{HD 111005}

Solano et al. (2001) gave the spectral type hF0mA3 V, with abundances showing only mild $(\sim 0.3 \mathrm{dex})$ metal deficiency, but only Fe and Ti were measured. Kamp et al. (2001) added $\mathrm{Ca}$, finding $[\mathrm{Ca} / \mathrm{H}]=-0.40$ with an LTE analysis. Paunzen et al. (2001) classified this star as kA3hF0mA3 V LB. Composite spectrum reported by Gerbaldi et al. (2003). Recorded as an (uncertain) Am star in the Renson \& Manfroid (2009) catalogue with $\mathrm{kA} 4 \mathrm{mF} 2$. Our new spectrum fixes the spectral type at F2 V kA5mA5 $\lambda$ Boo?, where the late hydrogen line type necessitates abundances of $\mathrm{C}$ or $\mathrm{O}$ to distinguish this object from the intermediate Pop. II stars. Recommendation: probable member.

\subsection{HD 111164}

The first spectral types assigned to this star were as an early A star: A3 V (Appenzeller 1967) and A3 Vn (Jackisch 1972), while the first classifiers to assign a $\lambda$ Boo classification were Abt \& Morrell (1995) with 'A3 Vp(Lam Boo)'. We obtained a new spectrum and saw a normal A3 IV-V star. Unfortunately, HD 111164 does not have wide coverage in the $\lambda$ Boo literature and an abundance analysis is lacking, though a high $v \sin i$ value $\left(175 \mathrm{~km} \mathrm{~s}^{-1}\right.$, Abt \& Morrell 1995; $191 \mathrm{~km} \mathrm{~s}^{-1}$, Royer et al. 2002) might preclude one. The high $v \sin i$ might also have swayed Abt \& Morrell (1995) towards a mild $\lambda$ Boo classification. Hauck \& Mermilliod (1998) took $u v b y \beta$ measurements for this star, and Johnson $B$ and $V$ values are available. $B-V, b-y, \beta$ and $c_{1}$ collectively agree on a dwarf near A4. However, an enhanced $m_{1}$ index indicates strong metals, near A7. Recommendation: non-member.

\subsection{HD 111604}

Various consistent sources of $u v b y \beta$ photometry suggest this is a star near A7, where the $m_{1}$ index suggests the star is metal weak. Hence, the IUE proposal (Faraggiana 1990) with which data were obtained under the title 'The Lambda Boo Stars' before this star was confirmed as a class member. Renson et al. (1990) adopted this star as an uncertain member of the class, but Weiss et al. (1994) subsequently criticised this classification because the latter authors mistakenly associated HD 111604 with the Nova DQ Her. Based on the UV criteria for membership that were established by Faraggiana et al. (1990), Gerbaldi \& Faraggiana (1993) admitted this star to the $\lambda$ Boo group. Abt $\&$ Morrell (1995) 
independently classified this star as 'A5 Vp lambda Boo'. Andrievsky et al. (2002) carried out an LTE abundance analysis, finding $[\mathrm{C} / \mathrm{H}]=-0.25$ and metal deficiencies of 1 dex, confirming its $\lambda$ Boo status. A spectral type of ‘A8 Vn kA1.5 mA1 $\lambda$ Boo' was given by Griffin et al. (2012), who, like Stütz \& Paunzen (2006), refuted the claims that this star's spectrum is a composite, made by Gerbaldi et al. (2003) and Faraggiana et al. (2004). Recommendation: member.

\subsection{HD 111786 (HR 4881)}

Andersen $\&$ Nordstrom (1977) provided the first $\lambda$ Boo classification, but with a slightly contradictory statement: 'probably $\lambda$ Boo star: neutral lines strong, Ca II K and Mg II $\lambda 4481$ weak'.

There is a lot of evidence in favour of membership in the $\lambda$ Boo group. Baschek et al. (1984) noted moderate $\lambda$ Boo characteristics in the UV from low-resolution IUE spectra; these characteristics were confirmed and emphasised by Faraggiana et al. (1990). Maitzen \& Pavlovski (1989a) included this star in their $\Delta a$ photometry study, wherein it lies clearly among the $\lambda$ Boo population. The many investigations before 1990 led Renson et al. (1990) to include HD 111786 as a definite member of the class. Stürenburg's (1993) LTE abundance analysis showed metal depletions of -1.5 dex and a nearly solar C abundance; he calculated NLTE corrections for each element which revises the metal weakness to around -1.3 dex. It was independently classified as $\lambda$ Boo by Abt $\&$ Morrell (1995). We note that HD 111786 did not appear in the paper version of Paunzen's (2001) 'new and confirmed' list of $\lambda$ Boo stars because it was observed as a known $\lambda$ Boo (i.e. as a comparison star), but it is there in the online data in the second paper of that series.

Gray (1988) classified HD 111786 as 'A1.5 Va' $\lambda$ Boo', adding that $\lambda 4481$ was very weak compared to the metallicline spectrum, and that a weak, narrow, absorption core could be seen in the $\mathrm{Ca}$ II $\mathrm{K}$ line, which he attributed to circumstellar material. With higher resolution spectra, Holweger \& Stuerenburg (1991) described that 'weak, narrow, absorption core' as 'extremely conspicuous', as illustrated in their figure 3. Narrow absorption components were also noted for $\mathrm{Na} \mathrm{D}$ lines. Holweger \& Rentzsch-Holm (1995) noted the radial velocity of the circumstellar feature at $\mathrm{Ca}$ II $\mathrm{K}$ line to vary with time. In addition to the Ca II K line feature, Gray (1988) also noted peculiar hydrogen lines, which Iliev \& Barzova (1998) later investigated to show substantial residuals in the core of the Balmer lines.

Both the circumstellar features and the peculiar hydrogen lines have been explained as arising from an SB2 system (Faraggiana, Gerbaldi, \& Burnage 1997), where the primary is a broad lined and the secondary a narrow-lined A star. Faraggiana et al. (2001b) discovered that HD 111786 is, in fact, a multiple system having (probably) five members, as illustrated using the $\mathrm{O}$ I 7772-7775 feature in their figure 15. It appears to be composed of one broad-lined star, and four narrow-lined stars each having similar physical param- eters (luminosity and temperature). We acknowledge their statement that '[i]f we accept the definition of $\lambda$ Boo stars as single objects with peculiar atmospheric abundances then the classification of this complex object as a $\lambda$ Boo star must be definitely rejected'.

However, we are not ready to reject this classic $\lambda$ Boo star just yet. Let us consider the $\mathrm{Ca}$ II $\mathrm{K}$ line, for which the 'extremely conspicuous' absorption feature remains conspicuous in the binary hypothesis. The Ca II K line, for late-A stars and especially for early-F stars, is intrinsically broadno amount of slow rotation produces a narrow absorption component. We also look to the recent paper by Griffin et al. (2012), which rejects the composite-spectrum hypothesis as producing the $\lambda$ Boo phenomenon. They state that $\lambda$ Boo stars cannot be dismissed by 'that route'.

It is, incidentally, a 6-mmag pulsator with a frequency of $30 \mathrm{~d}^{-1}$ (Kuschnig, Paunzen, \& Weiss 1994a) that was later found to be multiperiodic (Paunzen et al. 1998b) and pulsating non-radially (Bohlender et al. 1999). If the pulsations can be shown to originate in one star, asteroseismic modelling may be able to shed light on the metal weakness. Key physical parameters are available (Iliev \& Barzova 1995) to facilitate this.

We obtained a new spectrum for this work, and classify it as F0 V kA1mA1 $\lambda$ Boo. It looks extremely metal weak and like a classic $\lambda$ Boo star. Mg II 4481 is barely a notable feature of the spectrum. $\mathrm{H}$ cores are slightly weak.

Recommendation: member.

\subsection{HD 111893}

HD 111893 is designated an uncertain member in the Renson et al. (1990) catalogue. Rapid rotation was noted in the spectral type (A5 IV-Vnn) given by Gray \& Garrison (1989b). Not identified as $\lambda$ Boo by Abt \& Morrell (1995), either. IRAS infrared excess detected (King 1994). It is not clear why Renson et al. (1990) ever considered this a $\lambda$ Boo candidate. Recommendation: non-member.

\subsection{HD 112097}

Classified as 'F0 Vp(Boo, met:A7) by Abt \& Morrell (1995), but the Skiff (2013) catalogue states it has been classified as Am: in the past. It is an Am star in the Renson \& Manfroid (2009) catalogue (Ca II K line: A6, metal lines F1), but inspection of $u v b y \beta$ photometry suggests hydrogen lines of about F0 with substantial metal weakness. Gray et al. (2001) gave a spectral type of $\mathrm{kA} 7 \mathrm{hF} 0 \mathrm{mF} 0(\mathrm{~V})$, supporting an Am: type. Recommendation: non-member.

\subsection{HD 113848}

Cowley \& Bidelman (1979) gave the classification F3 pec with the note ' $\mathrm{H} \sim \mathrm{F} 8$; $\mathrm{Ca}$ II $\sim \mathrm{F} 0$; no $G$ band; metals $\sim \mathrm{F} 3$ '. The fact that this is a known double star with sub-arcsec separation (WDS, Mason et al. 2001) could explain the peculiar 
spectrum. Designated as uncertain in the Renson et al. (1990) catalogue. IRAS infrared excess detected (King 1994). SIM$\mathrm{BAD}[\mathrm{Fe} / \mathrm{H}]$ values show only mild deficiency. Probably too late to be a $\lambda$ Boo star. A new and preferably high-resolution spectrum might provide a definitive assessment. Recommendation: non-member.

\subsection{HD 114879}

Designated as uncertain in the Renson et al. (1990) catalogue. Classified by Paunzen et al. (2001) as A3 V, with no comment suggesting a $\lambda$ Boo character, when they were looking for $\lambda$ Boo stars. Thus, we might assert it is not a $\lambda$ Boo star. Hauck \& Mermilliod's (1998) $u v b y \beta$ data show agreement in the $\beta$ and $b-y$ indices that this star is about A5/6, and the $c_{0}$ index matches A6 for a luminosity class $\mathrm{V}$ object. Then, the $m_{1}$ index suggests only mild metal weakness of 12 spectral subclasses. Reinvestigation of spectrum advised. Recommendation: non-member.

\subsection{HD 114930}

Designated as uncertain in the Renson et al. (1990) catalogue. Classified by Paunzen et al. (2001) as F0 IV, with no comment suggesting a $\lambda$ Boo character, when they were looking for $\lambda$ Boo stars. Thus, as for HD 114879, we might assert it is not a $\lambda$ Boo star. However, inspection of the Hauck $\&$ Mermilliod (1998) $u v b y \beta$ photometry suggests hydrogen lines of about F0/1 but metals of strength about A1. We have obtained and classified a new spectrum of this star, which warrants its rejection: F1 Vs, definitely not metal weak. The Ti/Fe II 4172-9 blend clearly points to luminosity class V. Recommendation: non-member.

\subsection{HD 118623}

Abt \& Morrell (1995) classified HD 118623 as 'F0 Vp(Lam Boo)n' with $v \sin i=190 \mathrm{~km} \mathrm{~s}^{-1}$. Classified as F0 Vnn kA8 by Gray et al. (2001), and Gray recommended it be dropped from the $\lambda$ Boo class for this reason, that is, for very rapid rotators $\lambda$ Boo classifications should be treated with skepticism. Previously classified by Jaschek \& Jaschek (1980) as 'UV: abn. CrEuSr ... MK: A7p ( $\mathrm{Sr}, \mathrm{Cr}) \ldots$ notes: $v \sin i$ $=204 \mathrm{~km} \mathrm{~s}^{-1}$. Others: A7 III'. HD 118623 has one close companion at 1.8 arcsec east, which is 2 mag fainter (WDS, Mason et al. 2001). Gerbaldi et al. (2003) noted 'composite, Hipparcos' and this candidate was therefore described as a $\lambda$ Boo star with a composite spectrum by Faraggiana et al. (2004) because it is less than 2 arcsec from another star that is less than 2.2 mag fainter. In this work, we have inspected the UV spectrum against the $\lambda$ Boo criteria in the UV given by Faraggiana et al. (1990); it does not have a $\lambda$ Boo character. Recommendation: non-member.

\subsection{HD 119288}

Uncertain member in the Renson et al. (1990) catalogue. F3 Vp star with $[\mathrm{Fe} / \mathrm{H}]=-0.46, T_{\text {eff }}=6600 \mathrm{~K}$ and $\log g=$ 4.03 (Cenarro et al. 2007). Classified as 'F5 V ((metalweak))' by Gray et al. (2001). IRAS infrared excess detected (King 1994). Reported long-period roAp star (Matthews \& Wehlau 1985), where its spectrum was described as being atypical for a magnetic Ap star since $\mathrm{Ca}$ is enhanced and metals slightly underabundant, but follow-up observations could not confirm variability (Heller \& Kramer 1988). No particular evidence in favour of $\lambda$ Boo membership except slight metal weakness; a high-resolution spectrum should easily confirm our recommendation for rejection. Recommendation: non-member.

\subsection{HD 120500}

Presented as a new $\lambda$ Boo star by Paunzen $\&$ Gray (1997), who gave a spectral type of 'kA1.5hA5mA1.5 V ( $\lambda$ Boo)' and a comment 'mild $\lambda$ Bootis star'. NLTE abundances for $[\mathrm{N}],[\mathrm{O}]$, and $[\mathrm{S}]$ are $-0.30,+0.19$, and -0.13 , respectively (Paunzen et al. 1999b; Kamp et al. 2001). LTE abundances for metals (Andrievsky et al. 2002) suggest only a mild $\lambda$ Boo character, which is why Andrievsky et al. described their results as 'ambiguous' in evaluating this star's $\lambda$ Boo membership. We accept HD 120500 as a mild $\lambda$ Boo star. Recommendation: member.

\subsection{HD 120896}

Classified as kA6F0mA6 V LB in the Paunzen et al. (2001) spectroscopic survey and given in the results paper (Paunzen $2001)$ as a new $\lambda$ Boo star. Strömgren indices from Hauck $\&$ Mermilliod (1998) $(b-y=0.166, \beta=2.674)$ agree with the hydrogen line type, for which $m_{1}(=0.150)$ reveals a marked metal weakness. This is a mild $\lambda$ Boo star. Recommendation: member.

\subsection{HD 123299}

This star, $\alpha$ Dra, was adopted by Gray \& Garrison (1987) as one of their A0 III low $v \sin i$ standards, and is upheld as such by Gray \& Corbally (2009). This is sufficient reason to reject the star from the $\lambda$ Boo group. Uncertain designation in the Renson et al. (1990) catalogue. Rejected by Paunzen et al. (1997) because of its identification by Gray \& Garrison (1987) as a non- $\lambda$ Boo star. Recommendation: non-member.

\subsection{HD 125162}

This is $\lambda$ Boo itself, the group prototype. Obviously a member. The spectral type on Gray's website is 'A3 Va kB9mB9 Lam Boo'. Recommendation: member. 


\subsection{HD 125489}

Abt \& Morrell (1995) gave the spectral type 'F0 Vp(Boo, met: A5)'. Gerbaldi et al. (2003) gave the remark: 'inconsistent UV flux (bin.?)'. RVs at different wavelengths in Griffin et al. (2012) show discrepancies, but they described it as 'a single, late-A dwarf or sub-giant with slightly enhanced metal abundances of 0.20 dex'. The WDS does list a companion at 1.8 arcsec, but it is $6.5 \mathrm{mag}$ fainter. So although binary contamination is not an issue, metal enhancement means it is not $\lambda$ Boo star. Recommendation: non-member.

\subsection{HD 125889}

One of the confirmed $\lambda$ Boo stars of Paunzen (2001) with a spectral type 'kA4hF2mA4 V ( $\lambda$ Boo)'. We confirm the weak 4481 line in our spectrum, with the type $\mathrm{F} 1 \mathrm{Vs} \mathrm{kA} 4 \mathrm{~mA} 4$ ( $\lambda$ Boo), but given the late hydrogen line type, an abundance analysis is required to distinguish from an intermediate Pop. II star. Recommendation: probable member.

\subsection{HD 128167 ( $\sigma$ Boo)}

An uncertain member of the $\lambda$ Boo group according to the Renson et al. (1990) catalogue. A fairly late $\lambda$ Boo candidate, with $B-V=0.36$, classified by Gray et al. (2001) as 'F4 V kF2mF1'. Paunzen et al. (1997) rejected it for this reason. Abundances from Adelman et al. (1997) show metal deficiencies of about 0.4 dex, but the same is true for [C], hence the $\lambda$ Boo pattern is not exhibited. IRAS infrared excess detected (King 1994). NLTE abundances would help to more firmly reject this candidate. Recommendation: nonmember.

\subsection{HD 130158}

Misclassified according to the Renson et al. (1990) catalogue. Called 'A0 IIIp(Lam Boo)' later by Abt \& Morrell (1995). Identified as an Ap star: 'A0 II-IIIp (Si)' in the observational paper of Paunzen et al. (2001) and B9 Si in the Renson \& Manfroid (2009) catalogue. Physical parameters of this magnetic Ap star can be found in Wraight et al. (2012). Recommendation: non-member.

\subsection{HD 130767}

Paunzen et al. (2001) gave the spectral type 'A0 Va $\lambda$ Boo' in their observational paper, with the additional comment in the results paper that this star is very similar to $\lambda$ Boo itself (Paunzen 2001). Key physical parameters are available (Paunzen et al. 2002a). No published high-resolution spectroscopy/abundance analysis is available. Recommendation: member.

\subsection{HD 138527}

Abt \& Morrell (1995) classified HD 138527 as 'B9.5 Vp(Boo: Ca, $4481 \mathrm{wk}$ )' but were unable to determine $v \sin i$. Adaptive optics observations revealed this star to have a companion (Gerbaldi et al. 2003), but RVs are constant within the errors (table 3 of Griffin et al. 2012). The latter authors also wrote 'metals are sub-solar by 0.8 dex and its high rotational velocity [135 $\left.\mathrm{km} \mathrm{s}^{-1}\right]$ accentuates the weakness of its lines', and that an interstellar Ca II K line feature was seen (see their figure 7). They acknowledge the double star nature claimed by Gerbaldi et al. (2003), but assert the spectra show no RV shift or other signature of an SB2 system, and that the magnitude difference $(\Delta m=3.2 \mathrm{mag}$ in the IR, and greater in the blue) is too large for the 'contaminating' star to veil the lines of the brighter component. An NLTE abundance analysis of volatile elements, if possible given the $v \sin i$, would arbitrate membership into the $\lambda$ Boo group. Recommendation: probable member.

\subsection{HD 139614}

Although Acke \& Waelkens (2004) found an overall metal deficiency, they did not see selective $\lambda$ Boo depletion. Folsom et al. (2012) did, however, and describe this star as having a 'clear $\lambda$ Boo pattern'. Inspection of their abundances show this is very mild. Volatile and refractory elements are, on average, about 0.1 and 0.5 dex below solar, respectively. $\mathrm{Mg}$ is $0.23 \mathrm{dex}(=3 \sigma)$ below solar. Our spectral type is F0 Vse kA4mA6 ( $\lambda$ Boo). The $\lambda 4481$ weakness is mild, and so we accept this as a very mild $\lambda$ Boo star. The WDS catalogue (Mason et al. 2001) lists a companion at 1.4 arcsec, but $\Delta m=3.5 \mathrm{mag}$, so any contamination is small. No $I U E$ spectrum is available to investigate the UV properties. Recommendation: member.

\subsection{HD 141569}

Gray \& Corbally (1998) grouped this star into a set that were metal weak, without displaying all $\lambda$ Boo features, and commented that these stars are probably metal weak due to accretion of metal-poor material. Folsom et al. (2012) 'found $\lambda$ Boo peculiarities' in this star through an abundance analysis. Although $\mathrm{C}$ is approximately solar, $\mathrm{N}$ and $\mathrm{O}$ are underabundant by 0.3 dex each ( 1 and $3 \sigma$, respectively), $\mathrm{Mg}$ is underabundant by $0.4 \mathrm{dex}(4 \sigma)$, and $\mathrm{Fe}$ and $\mathrm{Ti}$ are both 0.7 dex $(2 \sigma)$ underabundant. This is consistent with a mild $\lambda$ Boo star. Our spectral type is $\mathrm{A} 2 \mathrm{Ve} \mathrm{kB} 9 \mathrm{mB} 9 \lambda$ Boo. We noted infilling of $\mathrm{H} \beta, \mathrm{H} \gamma$ and $\mathrm{H} \delta . \lambda 4481$ is weak, and the spectrum is generally metal weak. The WDS catalogue (Mason et al. 2001) paints a picture of multiplicity: companions of estimated masses $0.45,0.22$, and $1 \mathrm{M}_{\odot}$ for components $\mathrm{B}$, $\mathrm{C}$, and D were determined by Feigelson, Lawson \& Garmire (2003). Their separations from HD 141569 A are 7.6, 8.1, and 1.5 arcsec, respectively. Recommendation: member. 


\subsection{HD 141851}

Identified as a $\lambda$ Boo star by Abt (1984a) and included in the Renson et al. (1990) catalogue. Paunzen et al. (1999b) found NLTE $[\mathrm{C}]$ and $[\mathrm{O}]$ abundances of -0.81 and -0.21 , respectively, and $v \sin i$ in excess of $200 \mathrm{~km} \mathrm{~s}^{-1}$. Kamp et al. (2001) did an LTE abundance analysis and found $[\mathrm{Ca} / \mathrm{H}]=-1.30$ dex but reliable NLTE abundances for $\mathrm{N}$ and $S$ could not be determined. Paunzen et al. (2001) gave a spectral type 'A2 Van', but no mention of $\lambda$ Boo. Paunzen et al. (2002b) argued this star is misclassified (that is, not a $\lambda$ Boo star), citing the results paper of Paunzen (2001). No mention of this star is actually made in the latter paper, but Paunzen et al. (2001) did clearly state that this is not a $\lambda$ Boo star. Andrievsky et al. (2002) found $[\mathrm{Fe} / \mathrm{H}]=-0.70$ and $[\mathrm{Si} / \mathrm{H}]=-0.65$ with $[\mathrm{Na} / \mathrm{H}]=+0.60$ dex, but were not able to decide if this star was a $\lambda$ Boo star. Indeed, the overall picture from abundance analyses is that of either a metal poor or mild $\lambda$ Boo star. Gerbaldi et al. (2003) recalled the existence of a companion at 0.1 arcsec and claimed to measure a magnitude difference but did not provide it. A comment of 'detection of a star three times fainter $0.15 \operatorname{arcsec}$ (east)' is given in their adaptive optics summary table (their table 3 ). The contamination is discussed in more detail by Faraggiana et al. (2004). The WDS catalogue (Mason et al. 2001) lists a separation of $0.4 \operatorname{arcsec}$ and $\Delta V=2.6 \mathrm{mag}$.

In our own spectrum of this star, we note that the $\mathrm{H}$ lines do not match particularly well at any type, but the spectrum is consistent with a normal A2 IVn star. The $\mathrm{H}$ line cores are shallower than in $\beta$ Ser (the A2 IVn standard), even though $v \sin i$ is about the same, but the match is otherwise good. The $\lambda 4481$ line is still weak, but in rapidly rotating early $\mathrm{A}$ stars, $\lambda 4481$ does look weak (Gray 1986).

In light of the new spectral type, the mild metal underabundances, the non-solar volatile element abundances, the rejection from earlier catalogues, and the complexities introduced by the double/multiple star nature, we reject this candidate. Recommendation: nonmember.

\subsection{HD 142666}

Has a spectral type of A8 Ve (Mora et al. 2001). Folsom et al. (2012) found $\mathrm{C}, \mathrm{N}$, and $\mathrm{O}$ to have solar abundance while the Fe-peak elements are 0.5 dex below solar (Fe itself is at $-0.25 \mathrm{dex})$, concluding this is a weak $\lambda$ Boo star. We reobserved this target, whose spectrum suggests it is a shell star: 'F0 Vs shell?', and perhaps a spectrum variable. In our spectrum, the $\mathrm{H}$ lines have very strong cores and the metal lines have inconsistent morphologies with one another. Despite the mildly anomalous abundances reported by Folsom et al. (2012), the morphology is consistent with a shell star but not a $\lambda$ Boo star, and so in the absence of a UV spectrum we are forced to reject this star. Recommendation: nonmember.

\subsection{HD 142703}

Suggested candidate by Hauck (1986) and listed in the Renson et al. (1990) catalogue. PHL with 7200-K cores but 7700K wings (Iliev \& Barzova 1993b). Gray \& Corbally (1993) classified the star as 'kA1hF0mA1 Va $\lambda$ Boo'. Key physical parameters are available (Iliev \& Barzova 1995). Heiter (1998) listed a cool $T_{\text {eff }}$ of $7000 \mathrm{~K}$ and found $\log g=3.7$, $[Z]=-1.5$ and $v \sin i=100 \pm 10 \mathrm{~km} \mathrm{~s}^{-1}$. Iliev \& Barzova (1998) saw emission in $\mathrm{H} \gamma$ (see their figure 1). NLTE abundances of $[\mathrm{C}],[\mathrm{N}],[\mathrm{O}]$, and $[\mathrm{S}]$ are $-0.52,-0.60,-0.19$, and -0.52 , respectively (Paunzen et al. 1999b; Kamp et al. 2001), and the latter authors also measured an LTE Ca abundance of -1.40 dex. For refractory metals ( $\mathrm{Mg}$ through to $\mathrm{Zn}$ ) abundances are on the order -1 dex (Solano et al. 2001; Heiter, Weiss, \& Paunzen 2002). No remarks to justify rejection in Gerbaldi et al. (2003), but Faraggiana et al. (2004) claimed it is a probable multiple system with no further comment. Recommendation: member.

\subsection{HD 142944}

Described in Paunzen et al. (2002b) as a pulsating $\lambda$ Boo star with $v \sin i=180 \mathrm{~km} \mathrm{~s}^{-1},[Z]=-0.91$ and a period of $8.1 \mathrm{~d}^{-1}$, but the low $\mathrm{Q}$ value on the pulsation implies this is not the fundamental radial mode. Key physical parameters available from Iliev \& Barzova (1995) and Paunzen et al. (2002a) are in agreement. The MK type of A0 V (Houk 1982) is much earlier than the $B-V$ measurement of $0.17 \mathrm{mag}$ (Slawson, Hill \& Landstreet 1992) would suggest. No argument in favour of $\lambda$ Boo membership, based on spectra, can be found in the literature. No $u v b y \beta$ photometry exists. Our conclusion is that its entry was a mistake (typo) on the part of Paunzen et al. (2002b) and Paunzen et al. (2002a), and really they meant to write HD 142994. The latter is confirmed as a $\lambda$ Boo star in the following subsection. Given the faintness of HD 142944, and lack of published Strömgren photometry, we conclude it did not fall within Paunzen's survey boundaries. Paunzen has confirmed in a private communication that HD 142944 was a typo in the data tables (it should have read HD 142994), but the main text remains accurate. Recommendation: non-member.

\subsection{HD 142994}

Gray (1988) claimed this is a genuine $\lambda$ Boo star with a spectral type 'A3 Va $\lambda$ Boo ... PHL' and a note: 'the $\mathrm{K}$ line and metallic-line spectrum are similar in strength to those of the A3 IV standard $\beta$ Eri, but $\lambda 4481$ is very weak, but present. The hydrogen lines have weak cores, with strengths similar to those of F0 stars, but with broad and very shallow wings. Moderate $v \sin i$. Photometry from Olsen (1983) who reported possible variability in the $c_{1}$ index. Photometry of this star in May 1987 shows similar residuals in $c_{1}$ '.

Key physical parameters are available (Iliev \& Barzova 1995). Paunzen et al. (1999a) could only gather LTE 
abundances for three elements: $[\mathrm{Mg} / \mathrm{H}]=-0.2,[\mathrm{Ti} / \mathrm{H}]=$ -1.5 , and $[\mathrm{Fe} / \mathrm{H}]=-1.5$ dex. High $v \sin i\left(180 \mathrm{~km} \mathrm{~s}^{-1}\right.$, Paunzen et al. 2003) inhibits abundance analyses, but nonetheless those authors recorded the presence of circumstellar gas, citing Bohlender et al. (1999). No evidence of a companion (e.g. Solano et al. 2001; Gerbaldi et al. (2003)). From existing and new spectra, we determine this is a bona fide $\lambda$ Boo star with spectral type F0 $\mathrm{V}$ kA3mA3 $\lambda$ Boo. Recommendation: member.

\subsection{HD 143148}

Given as ‘ $\lambda$ Boo(?)’ by Paunzen, Weiss, \& Kuschnig (1996). Paunzen et al. (2002b) argued this star to have been misclassified, since Paunzen et al. (2001) found no $\lambda$ Boo characteristics, giving a classification of A7 IVn. We suspect this star was originally a $\lambda$ Boo candidate sometime after Olsen (1979) published Strömgren indices. He only identified two $\lambda$ Boo candidates, and this was not one of them. Rather, it was identified as an FHB star. But if one studies carefully the $u v b y \beta$ photometry available in the literature (Hauck \& Mermilliod 1998), one can infer hydrogen strengths near F0 but metal strengths near A0. Given the lack of evidence in favour of a $\lambda$ Boo classification, we reject the star for now, but it would be worthwhile to obtain a spectrum. Recommendation: non-member.

\subsection{HD 144708}

Abt \& Morrell (1995) classified HD 144708 as B9 Vp(Lam Boo)nn with a $v \sin i$ measurement of '255:' i.e. given with uncertainty. Not discredited by Gerbaldi et al. (2003), despite the 'essential note' on SIMBAD that this object includes another component. Faraggiana \& Bonifacio (1999) pointed out the duplicity of this star, with $P_{\text {orb }}=4.02 \mathrm{~d}$. We find the high $v \sin i$ for what must be a very strongly tidally braked system to be suspicious (see discussion in Murphy 2014, Ch 2). Highly discordant $u v b y \beta$ photometry. No good evidence in favour of $\lambda$ Boo membership. Recommendation: non-member.

\subsection{HD 145782}

Of the five spectral types available in the Skiff (2013) catalogue, not one suggests $\lambda$ Boo membership. Indeed, Paunzen et al. (2001) classified the star as A3 V, and they were specifically looking for $\lambda$ Boo stars. This is probably why Paunzen et al. (2002b) rejected this star, which was labelled as ' $\lambda$ Boo(?)' by Paunzen et al. (1996). Recommendation: non-member.

\subsection{HD 148638}

Solano et al. (2001) had this target in their sample, but noting it was a binary they chose not to derive the abundance pattern. In fact, the separation is $21.9 \operatorname{arcsec}$ (WDS, Mason et al. 2001) so there should have been no problem. Kamp et al. (2001) did, however, proceed to analyse the spectrum and determined supersolar $\mathrm{N}$ and $\mathrm{S}$ abundances using NLTE, along with $[\mathrm{Ca} / \mathrm{H}]=-1.20$ using LTE. Their figure 2 does appear to show large residuals after fitting the synthetic spectrum, possibly due to the binarity. Paunzen et al. (2001) classified the star as ' $\mathrm{hA} 7 \mathrm{~mA} 2 \mathrm{~V}$ LB', where the wide visual binary nature is acknowledged as a note in the result paper. Metal abundances have not been determined. $u v b y \beta$ photometry (Oblak 1978) is available; the $b-y$ and $\beta$ indices suggest a late A star, but then the $m_{1}$ index suggests considerable metal weakness, and the $c_{1}$ index is high. From our new spectrum, we classify the star as $\mathrm{A}^{2} \mathrm{IV}^{-} \mathrm{n}(4481-w \mathrm{k})$, but note that the spectrum is otherwise normal. Unfortunately, the substantial rotation will hinder high-resolution spectroscopic analyses, but a new analysis that considers the possibility of a hotter and less peculiar star would perhaps shed more light on this system. Recommendation: uncertain member.

\subsection{HD 149130}

Classified as kA7hF0mA7 V LB by Paunzen et al. (2001), with the note in Paunzen (2001) that the spectrum is only mildly metal weak and that the Michigan Catalogue gives the spectral type as A8wl. This star is mentioned in many papers on weak-lined stars. Abundance analysis desired to confirm membership.Recommendation: probable member.

\subsection{HD 149303}

Appears in the Renson et al. (1990) catalogue. NLTE $[\mathrm{O} / \mathrm{H}]=$ -0.14 and LTE $[\mathrm{Ca} / \mathrm{H}]=-0.50$ (Kamp et al. 2001). Abundance analysis in Paunzen (2000) exists only for $\mathrm{Mg}(-0.2)$, $\mathrm{Ti}(-1.3), \mathrm{Fe}(-0.9)$, and $\mathrm{Cr}(-0.5 \mathrm{dex})$, indicating a mild $\lambda$ Boo star. Classified as A3 IV-V (wk 4481) by Paunzen et al. (2001), but not as $\lambda$ Boo, hence it was rejected by Paunzen et al. (2002b). Binarity not recorded by Gerbaldi et al. (2003) even though it was detected as an SB system by Paunzen et al. (1999b). The separation is large, at 16.3 arcsec (WDS, Mason et al. 2001). Gray et al. (2001) gave the secondary a spectral type of F9 V, but did not observe the primary. We observed both stars for this work. We give the spectral type A2 IV-Vn (normal) for the primary, and F9 V for the secondary. The secondary shows emission reversals in the $\mathrm{Ca}$ II $\mathrm{K}$ and $\mathrm{H}$ lines, and is therefore an active star. Age-activity relations will allow an age for the system to be determined. Recommendation: uncertain member.

\subsection{HD 153747}

The results paper of Paunzen (2001) lists the 'estimated' spectral type as hA7mA0 V LB, where its $\delta$ Sct variability is also noted; Desikachary \& McInally (1979) reported multiperiodicity in the light curve. Paunzen et al. (2002b) gave $[Z / H]=-0.86 \pm 0.20$. The pulsational nature of this star has led to wide coverage in the literature, but little appears to be 
said of its $\lambda$ Boo character. We obtained a new spectrum to rectify this, but the classification was difficult. The hydrogen lines fit best at A7, taking into account the enormous difference in line blanketing, but a match near $\mathrm{A} 1 \mathrm{~V}^{-}$cannot be excluded, although the core is a bit too deep and narrow for this match. In either case, the star is metal weak, and would be classified as $\lambda$ Boo under both interpretations. The photometry really does not help to decide between the two, because of the possibility of reddening, and the fact that a very metal-weak A7 star would be quite blue. The dominance of Ca I 4226 in its region and complete lack of Ti/Fe II 41729 Fe II 4233 argues for the cooler type. Our final spectral type is $\mathrm{A} 7 \mathrm{~V}$ kA0mA0 $\lambda$ Boo. Recommendation: member.

\subsection{HD 153808}

Abt \& Morrell (1995) gave the classification A0 IVp ( $\lambda$ Boo) with $v \sin i=50 \mathrm{~km} \mathrm{~s}^{-1}$, whereas Gray \& Garrison (1987) gave the classification $\mathrm{A} 0 \mathrm{IV}^{+}$. The star's duplicity (multiplicity) was discussed by Faraggiana et al. (2001b), where the contamination is demonstrated to be large. There is no evidence, other than a contaminated and uncorroborated classification by Abt \& Morrell (1995), to suggest we confer $\lambda$ Boo membership. Recommendation: non-member.

\subsection{HD 154153}

Renson et al. (1990) listed this star as misclassified, but it did not appear to be decisively dropped by others; Paunzen et al. (1997) rejected it because it is an evolved star, while the online data for the observational paper of Paunzen et al. (2001) has the spectral type 'A3 V LB', and the results paper (Paunzen 2001) gave the spectral type ' $\mathrm{hF0mA5}$ ( $\lambda$ Boo)'. In their investigation into the period-luminosity-colour relation in pulsating $\lambda$ Boo stars, Paunzen et al. (2002b) treated this star as a $\lambda$ Boo star and found it to be non-variable. We observed this target and give the spectral type F1 V kA3mA3 $\lambda$ Boo?, where the late hydrogen line type has prevented distinction between a $\lambda$ Boo star and a Pop. II star until NLTE abundances of volatile elements are available. The $\lambda 4481$ line is weak. Recommendation: probable member.

\subsection{HD 156954}

The remark for this star in Paunzen et al. (1997) reads 'Mg II too weak, otherwise normal metal spectrum', but Paunzen (2001) later gave the spectral type 'hF1mA5 V ( $\lambda$ Boo)' in his results paper. Solano et al. (2001) provided physical parameters. Metal deficiencies there are $\sim 0.5$ to $1.0 \mathrm{dex}$, with $<[\mathrm{Fe} / \mathrm{H}]>=-0.66$ dex. Gerbaldi et al. (2003) noted an inconsistent UV flux and suspected binarity. We have classified a new spectrum of this star as F1 Vs kA5mA4, with the comment ' $\mathrm{H}$ cores are shallow. Not Boo, just metal weak $-\lambda 4481 / \lambda 4383$ ratio is the same as at $F 1$, or perhaps very marginally weak, and at F1 this is a blend with Fe'. With the low $v \sin i$ of $51 \mathrm{~km} \mathrm{~s}^{-1}$ (Heiter et al. 2002) an
NLTE abundance analysis of the volatile elements would provide a decisive assessment. Recommendation: uncertain member.

\subsection{HD 159082}

Classified as A0 IVp $\lambda$ Boo by Abt \& Morrell (1995). Hauck et al. (1998) found a circumstellar Na I D signature, but noted this star was classified as a $\mathrm{HgMn}$ star (cf. entry in the Renson \& Manfroid 2009 catalogue as A0 HgMn) and should thus be rejected. Gerbaldi et al. (2003) said the UV flux was inconsistent and this star is a suspected binary; indeed, Bidelman (1988) described it as a 6.80-d binary and noted strong Hg II. Recommendation: non-member.

\subsection{HD 160928}

Listed in the Renson et al. (1990) catalogue. A comment in the Paunzen et al. (1997) consolidated catalogue of $\lambda$ Boo stars notes that $\mathrm{Mg}$ II is too weak, but the spectrum is otherwise normal. Paunzen et al. (2001) later classified the star as A2 IV (wk met), i.e. not as $\lambda$ Boo. This, in itself, is a good reason to exclude the star here. Fulfils the criteria of Faraggiana et al. (2004) for having a composite spectrum, because it is in the WDS Catalogue as having a separation of less than 2 arcsec from a star with a magnitude difference not larger than $2.2 \mathrm{mag}$. Indeed, this is evident in our classification spectrum, in that the $\mathrm{H}$ cores appear weak. We give the spectral type $\mathrm{A} 2 \mathrm{IV}^{-} \mathrm{n}$, and note a normal $\lambda 4481$ line, given the rapid rotation. Recommendation: non-member.

\subsection{HD 161223}

Studied among other pulsating $\lambda$ Boo stars by Gopka et al. (2007), where its chemical composition was provided for the first time and the authors described it as 'a new $\lambda$ Boo star'. However, it was already classified as a 'mild but bona fide $\lambda$ Boo star' by Gray \& Corbally (2002), with a spectral type of A9 V kA5 mA5 ( $\lambda$ Boo). It has, in the past, been classified as an SX Phe star (a population II $\delta$ Sct star), but the pulsation frequencies quoted by Gopka et al. (2007) are quite low for such an object. Furthermore, its proper motions

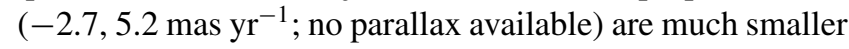
than one expects for a population II star. Gopka et al. (2007) found $\log g=3.3$, indicating this is quite an evolved $\lambda$ Boo star, and they state 'the classification of V2314 Oph as a $\lambda$ Boo type star seems reliable'. Clarification with a UV spectrum should be sought, and an abundance analysis is desirable. Recommendation: member.

\subsection{HD 161868}

Classified as ‘A0 Vp (4481 wk)n’ by Abt \& Morrell (1995), with $v \sin i=185 \mathrm{~km} \mathrm{~s}^{-1}$. Gray \& Garrison (1987) had earlier classified this as A0 Van, and Gray et al. (2003) classified this star as A1 Vn kA0mA0 with $T_{\text {eff }}=8951 \mathrm{~K}, \log g=4.03$ and 
$[M / H]=-0.81$. Hauck et al. (1998) reported an expanding circumstellar gas from the Ca II $\mathrm{K}$ line of this star (see also their figure $1 \mathrm{~h}$ ). Perhaps, it was the lack of ' $\lambda$ Boo' in the Gray $\&$ Garrison (1987) classification led Paunzen et al. (1997) to reject this star from the $\lambda$ Boo group when making their consolidated catalogue. Recommendation: non-member.

\subsection{HD 168740}

Proposed as a 'good $\lambda$ Boo candidate' by Hauck (1986). Paunzen et al. (1999b) found NLTE [C] and [O] abundances of -0.42 and -0.03 , respectively. Solano et al. (2001) provided key physical parameters and gave a spectral type of $\mathrm{hA} 7 \mathrm{~mA} 2 \mathrm{~V}$ and $[\mathrm{Fe} / \mathrm{H}]=-0.73$. The abundance analysis in Heiter (2002) agrees with the $\sim 1$ dex underabundance of metals, and the abundance pattern was described there as being typical of $\lambda$ Boo stars. From our new spectrum, we give the spectral type A9 V kA2mA2 $\lambda$ Boo. Recommendation: member.

\subsection{HD 168947}

The results paper of Paunzen (2001) lists this as a new $\lambda$ Boo star with a spectral type of kA3hF0mA3 V $\lambda$ Boo. We confirm this spectral type with our new spectrum. In their analysis of the Rodríguez, López-González, \& López de Coca (2000) catalogue of $\delta$ Sct stars, Rodríguez \& Breger (2001) tabulated this star as a 20-mmag $\delta$ Sct star with an oscillation frequency of $17 \mathrm{~d}^{-1}$ (its variability was first detected by Paunzen, Weiss \& North 1994). Strömgren photometry from Gray \& Olsen (1991) supports the spectral type. Recommendation: member.

\subsection{HD 169009}

Spectral type of A1 V $\lambda$ Boo according to Abt \& Morrell (1995), but a normal A0 IV star in the Paunzen et al. (2001) observational data. Since they were looking for $\lambda$ Boo stars, and did not classify this as $\lambda$ Boo, we can assert for now that it is not a member. This assertion is apparently justified, as in their extensive notes on this star, Griffin et al. (2012) discussed the peculiarity of this star as 'B9 V He-wk', and presented no evidence supporting a $\lambda$ Boo classification. Recommendation: non-member.

\subsection{HD 169022}

Originally classified as ' $\lambda$ Boo or composite' by Slettebak (1975), but Gray (1988) argues misclassification. Faraggiana et al. (1990) confirmed this star meets none of the UV criteria for $\lambda$ Boo membership, except from a negative $\Delta m_{1}$ index. In the Renson et al. (1990) catalogue, it is listed as misclassified, and Gerbaldi et al. (2003) wrote the spectral type as 'A0 $\mathrm{II}^{-}(\mathrm{n})$ shell ... misclassified', where that spectral type is the one assigned by Gray \& Garrison (1987). High proper motion but small tangential velocity. Recommendation: non-member.

\subsection{HD 169142}

Paunzen et al. (2001) classified HD 169142 as 'F0 Ve'. Folsom et al. (2012) found 'a clear $\lambda$ Boo pattern of abundances' in this star. ' $\mathrm{C}$ and $\mathrm{O}$ are solar, and $\mathrm{S}$ is only slightly underabundant, while iron peak elements are underabundant by between 0.5 and 1 dex'. We have obtained two spectra of this target, which we classify as F1 V kA3mA3 and F1 V kA4mA5. Both spectra show shallow H cores, but neither has a typical $\lambda$ Boo morphology. The metal lines have inconsistent strength, being stronger in the violet than the blue. The shallow $\mathrm{H}$ cores could be from emission, though we see no clear emission lines, or due to binarity. Indeed, weak emission in the $\mathrm{H} \alpha$ (Dunkin, Barlow, \& Ryan 1997) and the O I 6300 line (Acke, van den Ancker, \& Dullemond 2005 ) are recorded. The former gave the classification A5 Ve, and noted 'an absence of any significant depletion of $\mathrm{Ca}$, Ti or Fe'. We infer HD 169142 is a spectrum variable. Recommendation: uncertain member.

\subsection{HD 170000}

A0 IIIp $(\lambda$ Boo) in the Abt \& Morrell (1995) catalogue, with $v \sin i=65 \mathrm{~km} \mathrm{~s}^{-1}$. Garrison \& Gray (1994) gave the spectral type 'kB9hB9HeA0 V (Si)', in agreement with the Renson \& Manfroid (2009) entry 'A0 Si'. HD 170000 was even used as an Ap standard in testing of the spectral classification software, MKCLASS (Gray \& Corbally 2014). The WDS catalogue (Mason et al. 2001) lists a companion at 0.5 arcsec with $\Delta V=1.4 \mathrm{mag}$. A probable orbital period of $27 \mathrm{~d}$ is given there. Recommendation: non-member.

\subsection{HD 170680}

Has a spectral type of A0 Van ( $\lambda$ Boo) NHL in Paunzen \& Gray (1997), with the additional note "classified as A0 Vp (Ca, Mg wk) by Abt (1984a), confirmed as $\lambda$ Boo star in the UV by Baschek \& Slettebak (1988)'. Gray has reclassified that spectrum for this work, as 'A0 Van kB9 ( $\lambda$ Boo)'. NLTE abundances for $[\mathrm{C}]$ and $[\mathrm{O}]$ show solar values, at -0.06 and -0.07 dex, respectively (Paunzen et al. 1999b). Abundance analysis of four metals shows mild weakness $(\sim 0.4 \mathrm{dex}$, Heiter 2002), but $v \sin i$ is recorded there as $200 \mathrm{~km} \mathrm{~s}^{-1}$, making abundance analysis difficult. Listed as a double star with 0.1 -arcsec separation in the WDS catalogue (Mason et al. 2001), but with no matching entry in the CCDM (Dommanget \& Nys 2002). Recommendation: member.

\subsection{HD 171948 A}

Classified as A0 Vp $\lambda$ Boo by Abt (1985). Has a spectral type from Paunzen \& Gray (1997) of A0 $\mathrm{Vb} \lambda$ Boo NHL. Gray has reclassified that spectrum for this work 
as 'A3 $\mathrm{Va}^{-} \mathrm{kB} 8.5 \lambda$ Boo', and commented this is an extreme $\lambda$ Boo star with a very weak $\lambda 4481$ line. Iliev et al. (2002) conducted a full abundance analysis on this binary system and determined that both stars are true $\lambda$ Boo stars, and that the system is quite young (10-100 Myr). They found $T_{\text {eff }}=9000 \pm 200 \mathrm{~K}, \log g=4.0 \pm 0.15$, and $v_{\text {mic }}=2 \pm 0.5 \mathrm{~km} \mathrm{~s}^{-1}$ for both components, with $v \sin i=15$ and $10 \mathrm{~km} \mathrm{~s}^{-1}$ for the $\mathrm{A}$ and $\mathrm{B}$ components, respectively. Each star has a mass listed there as $2.0 \pm 0.1 \mathrm{M}_{\odot}$. Recommendation: member.

\subsection{HD 171948 B}

See entry for HD 171948 A. Recommendation: member.

\subsection{HD 172167}

This star, Vega, was noted to have $\lambda$ Boo like abundances by Baschek $\&$ Slettebak (1988) and is listed as an uncertain member of the $\lambda$ Boo group in the Renson et al. (1990) catalogue. Section 6.5 of Holweger \& Rentzsch-Holm (1995) is dedicated to the discussion of Vega and $\beta$ Pic as $\lambda$ Boo stars, but for Vega this is largely focussed on its rotation velocity and inclination angle. It is listed there with the 'dusty, normal stars'. Its abundances are like those of a mild $\lambda$ Boo star, with $\mathrm{C}$ and $\mathrm{O}$ being solar while metals are about $0.5 \mathrm{dex}$ below solar (see Hekker et al. 2009 for a literature comparison). The question of whether Vega should be adopted was addressed by Ilijic et al. (1998). They decided it should be based on abundances. The metal weakness is also observed in the UV, but there is no published application of $\lambda$ Boo UV criteria to Vega. A cursory examination of one of many of the IUE spectra of Vega for this work showed no 1600$\AA$ flux depression, and a normal C/Al ratio. It thus remains unclear whether Vega should be accepted as a (mild) $\lambda$ Boo star. Recommendation: probable member.

\subsection{HD 174005}

Described as an $\mathrm{A} 0+\mathrm{A} 2$ spectroscopic binary in the online catalogue of Paunzen et al. (2001), it was given the spectral type A7 V kA2 mA2 $\lambda$ Boo by Gray et al. (2001), with the comment that it is a 'classic $\lambda$ Boo star'. Faraggiana et al. (2004) included HD 174005 in their list of $\lambda$ Boo stars with composite spectra, but the maximum separation is 37.9 arcsec (Solano et al. 2001; Mason et al. 2001), which is rather far for contamination to be an issue. We note from our own observations that this is the brightest star around, and all other noticeable but fainter stars within 1 arcmin have their own HD designations. Recommendation: member.

\subsection{HD 175445}

Spectral type of $\mathrm{hA} 5 \mathrm{~mA} 2 \mathrm{~V} \lambda$ Boo in the results paper of Paunzen (2001), with the note that this star is very similar to HD 120500. Not much information is available on this star;
Strömgren photometry indicates a spectral type around A2/3 with slightly weak metals, but reddening is unknown. There is no abundance analysis or UV spectroscopy to evaluate membership, but our new spectrum shows this is a normal star, to which we give the spectral type A1.5 Van. Recommendation: non-member.

\subsection{HD 177120}

Originally termed $\lambda$ Boo by Abt (1985), it was classified as 'A0.5 IV (shell)' in the online data of the observations paper of Paunzen et al. (2001), but importantly, not classified as $\lambda$ Boo, indicating the star should not be listed as a member of the $\lambda$ Boo group. This means it was 'downgraded' by them from $\lambda$ Boo, on account of its appearance in the earlier paper of Paunzen et al. (1997) as a $\lambda$ Boo star. This is explained by the (unpublished) classification notes that Gray wrote for that paper: 'The hydrogen lines are certainly peculiar; their profiles are best matched at A0/A1, but they are shallow in the cores, and the wings are roughly III-IV, although this is a function of the hydrogen line. For instance, at $\mathrm{H} \gamma$, the wings are best matched at III-IV, but at $\mathrm{H} \delta$ they are closer to IV, and at the higher [blue-ward] $\mathrm{H}$ lines they agree well with the A1 IV std. The discrepancy at the core is most pronounced for $\mathrm{H} \gamma$, but is present for all hydrogen lines. The $\mathrm{K}$ line has a curious profile, having broad wings and a shallow, pointed core. All of these details suggest a shell phenomenon. Thus, A0.5 IV (shell)'. We obtained a new spectrum, which shows exactly the same properties. Although HD 117120 is potentially an astrophysically interesting object worthy of spectroscopic follow-up, there is no evidence in favour of it being a $\lambda$ Boo star. Although we reject this candidate, an abundance analysis and would be valuable, as would a UV spectrum, but no IUE data exist for this star. Recommendation: non-member.

\subsection{HD 177756}

An Abt \& Morrell (1995) $\lambda$ Boo star that was suspected of having a composite spectrum on account of its variable RV (Faraggiana et al. 2004), yet RV measurements in the Griffin et al. (2012) debunking paper show constant RV within the errors. Those authors claimed any RV variation could be explained by rapid rotation and an oblate spheroidal stellar structure. The variations are not consistent with a SB, they said. They commented the spectrum is around B8 or B9, with a metal deficiency of about 0.5 dex. Spectral type given there was 'B9 IV (He-wk)', and it was noted that the Si lines looked normal.

We investigated this star's IUE spectrum for this work, but the results are inconclusive: the $\mathrm{C} / \mathrm{Al}$ ratio $(\lambda 1657 / \lambda 1671)$ would suggest a $\lambda$ Boo-like character in a cooler star, but at such an early spectral type the behaviour of these lines is unknown. Contrary to typical $\lambda$ Boo spectra in the UV, there is no 1600-A depression. Our spectral type based on a new blue-violet spectrum is of a normal B8.5 Vn star, though the 
$\lambda 4481$ line is weak. In reality, HD 177756 is just too early for a $\lambda$ Boo star. Recommendation: non-member.

\subsection{HD 179218}

Spectral type of A0 IVe (Mora et al. 2001). Quoting Folsom et al. (2012): 'We found a solar He abundance, abundances of $\mathrm{C}, \mathrm{O}$, and $\mathrm{Na}$ that are marginally enhanced relative to solar, and $\mathrm{N}$ and $\mathrm{S}$ abundances that are uncertain, but apparently enhanced relative to solar. Iron peak elements are depleted relative to solar by $\sim 0.5$ dex. We conclude that HD 179218 displays $\lambda$ Boo peculiarities'. Additional membership criteria, such as investigation of the UV spectrum, should be evaluated in light of the apparently mild peculiarity. Recommendation: probable member.

\subsection{HD 179791}

Uncertain classification in the Renson et al. (1990) catalogue. Hydrogen line profile was determined to be normal (Iliev \& Barzova 1993a), where they described the Mg II 4481 line to be strong. Described as misclassified by Paunzen et al. (2002b); indeed, Paunzen et al. (2001) gave it the spectral type A2 IV in their search for $\lambda$ Boo stars. Gerbaldi et al. (2003) said the UV spectrum showed solar abundances. Recommendation: non-member.

\subsection{HD 181470}

A 'significantly evolved star' according to table 1 of King (1994), and thus not pursued further by him. Narusawa et al. (2006a) called this star a 'metal-poor A star' and provided abundances from the literature showing metal deficiencies of between 0.4 and 0.8 dex alongside a sub-solar $\mathrm{C}$ abundance of -0.36 dex. Cowley (1991) made reference to this star's low $v \sin i$, putting it in a category alongside Vega as a suspected $\lambda$ Boo star seen pole-on. Abt \& Morrell (1995) did actually classify this star as 'A0 V', which is the same type as Vega. There is little evidence suggesting HD 181470 is a $\lambda$ Boo star. We therefore observed this star at classification resolution and found it to be distinctly non- $\lambda$ Boo in nature. We give the spectral type kA0hA1mA1 III-IVs, which is typical of a mild (hot) Am star. The $\lambda 4481$ line is normal. We also looked at an available $I U E$ spectrum, which showed this star is clearly not a $\lambda$ Boo star (and is perhaps a mild Am star); in $\lambda$ Boo stars the C I 1657/Al II 1670 ratio is usually enhanced, but in this star it is considerably weak. In our observations, we did not detect a binary nature, but the WDS catalogue (Mason et al. 2001) records a separation of 0.1 arcsec and notes that this is the spectroscopic binary U Sge. Ibanoğlu et al. (2006) determined the absolute parameters of this binary and gave spectral types B7.5 V + G4 III-IV. Recommendation: nonmember.

\subsection{HD 183007}

Erroneously dubbed a probable Am star by Abt \& Morrell (1995) with the classification 'Am (A1/A4:/A3)', but the early $\mathrm{K}$ and metal lines are actually consistent with the $\lambda$ Boo type. Entry in Paunzen et al. (2001) online data is just 'A1 IV'. Gray's notes on Paunzen's spectrum (private communication) read: 'Yes, this is a mild Lambda Boo star. . hA7mA3 V Lam Boo', yet strangely, Paunzen (2001) did not include it in his 'new and confirmed' list. Known binary in a 165-d orbit (Budaj 1997). Cucchiaro et al. (1979) noted that if their UV classification scheme is applied to this star, it has an Am character in the UV. High proper motion with a transverse velocity of $43.5 \mathrm{~km} \mathrm{~s}^{-1}$.

We assign the spectral type A8 Vs kA2(p)mA3 $\lambda$ Boo with the following comments: the $\mathrm{Ca}$ II $\mathrm{K}$ line is peculiar, with a narrow core but deep wings. This is common in magnetic Ap stars, but this spectrum shares no other properties with Ap stars. Instead, the peculiar $\mathrm{K}$ line is probably indicative of a composite spectrum. Indeed, we also note that the metal lines are stronger in the violet, where they have a strength of around A5, than in the blue where they are at A2 or A3. The metals are still quite weak compared to the $\mathrm{H}$ line type, especially the $\mathrm{Mg}$ II 4481 line, which is much weaker than for an A8 star. This indicates a $\lambda$ Boo nature.

In conclusion, the spectrum shows a variety of evidence consistent with the composite nature reported in the literature. HD 183007 could be a mild LB star with an Am companion, but that hypothesis is very difficult to evaluate.

Recommendation: uncertain member.

\subsection{HD 183324}

Gray (1988) classified the spectrum as A0 Vb $\lambda$ Boo NHL, with the note 'very weak $\mathrm{K}$ line, metallic-line spectrum featureless. $\lambda 4481$ is only marginally present. Hydrogen lines show very broad wings. Hydrogen line profiles are quite normal, except that the cores may be slightly weak for A0'. He has reclassified the star for this work to give a $\mathrm{K}$ line and metallic line type, as ' $\mathrm{kB} 9 \mathrm{hA} 3 \mathrm{mB} 9 \mathrm{Va} \lambda$ Boo', and commented that this is an extreme $\lambda$ Boo star. Renson et al. (1990) described this as one of only six 'definite' members of the $\lambda$ Boo class. One of the more extreme $\lambda$ Boo stars, with metal underabundances of 1 to 2 dex, with normal $\mathrm{C}$ and $\mathrm{Na}$ (Stürenburg 1993). Iliev \& Barzova (1993b) observed the hydrogen lines, confirming the NHL profile with $T_{\text {eff }}=9100 \mathrm{~K}$. Possibly variable, according to Kuschnig, Paunzen, \& Weiss (1994b). The IR study of Andrillat et al. (1995) resulted in the note ' $\mathrm{LB}$ shell? The $\mathrm{O}$ I lines are sharp, although not strong and the $\mathrm{C}$ I feature at $10684[\AA]$ is resolved into two components as in other shells'. Key physical parameters are available (Iliev \& Barzova 1995). Volatiles are near-solar $([\mathrm{C}]=-0.14,[\mathrm{~N}]=-0.30$, and $[\mathrm{S}]=-0.13$, Kamp et al. 2001). RV variable (Gerbaldi et al. 2003). Ambiguous evidence for circumstellar gas, and no indication of dust (Paunzen et al. 2003). Recommendation: member. 


\subsection{HD 184190}

No metal deficiency seen (Solano et al. 2001), contradicting the classification of A7/9s wl 'no metals' by Houk (1978). Thus, there is no good evidence for $\lambda$ Boo status, but a lack of metal deficiency at high resolution is sufficient to rule it out. Recommendation: non-member.

\subsection{HD 184779}

The spectral type in Paunzen's (2001) list of $\lambda$ Boo stars is $\mathrm{kA} 4 \mathrm{hF} 1 \mathrm{~mA} 4 \mathrm{~V}$ ( $\lambda$ Boo), where it is described as a "very cool $\lambda$ Boo star similar to HD 107223'. Houk (1978) gave the spectral type A3/5 IIp, with the qualifier 'probably early weak lined rather than luminous (narrow $\mathrm{H}$ lines)'. Appears in the paper on FHB stars by Philip \& Hayes (1983) but is labelled there as Pop. I. We have reobserved this star and classify it as F0.5 V kA5mA5 ( $\lambda$ Boo). We accept it as a mild $\lambda$ Boo star, but abundances are desirable for confirmation. Recommendation: member.

\subsection{HD 187949}

Identified by Hauck (1986) as a metal weak binary in a search for new $\lambda$ Boo stars, with spectral type A0 V + F8 IV, but that spectral type actually comes from the BSC (Hoffleit \& Jaschek 1982). Eclipsing binary of the Algol type (detached). Faraggiana et al. (1990) rejected the star because the only evidence of $\lambda$ Boo character they found was a slight discrepancy between the optical and UV spectral types, and on account of the binarity. Our new spectrum has shallow H cores, indicative of the binarity, and we give the spectral type A2 IV(n). Recommendation: non-member.

\subsection{HD 188164}

Paunzen et al. (1996) concluded this ' $\lambda$ Boo(?)' star was non-pulsating at the 4-mmag level. Spectral type of A3 V in Paunzen et al. (2001), i.e. they did not label this star $\lambda$ Boo, and they were specifically looking for $\lambda$ Boo stars. We have reobserved this target and give the spectral type A5 IV$\mathrm{V}$ kA2mA3 ( $\lambda$ Boo), but we note that there is degeneracy in the hydrogen line profile with $\mathrm{A} 7 \mathrm{~V}$ being an equal (and good) match. The $\lambda$ Boo peculiarity is only mild, and we recommend a full abundance analysis. Recommendation: member.

\subsection{HD 188728}

Spectral type of A1- in the Renson \& Manfroid (2009) catalogue, where the '-' character is used to denote an Am star. Temperature subclass is in agreement with other literature classifications. Lemke (1989) determined [Fe/H] was $\sim 0.5$ dex above solar. One of the few stars appearing above the line of normal stars in the $a$ vs. $b-y$ plot of Maitzen \& Pavlovski (1989b), whereas $\lambda$ Boo stars fall below that line.
Misclassified according to the Renson et al. (1990) catalogue. Recommendation: non-member.

\subsection{HD 191850}

Spectral type of kA4hF0mA4 V $\lambda$ Boo in the results paper of Paunzen (2001), where its entry in the Michigan Catalogue as A2 II/III was described as an indication of probable membership in the $\lambda$ Boo group. Atmospheric parameters listed by Paunzen et al. (2002b), including $[Z]=-0.96 \pm 0.30$. We reobserved the star, and give the spectral type F0 V kA3mA3 $\lambda$ Boo. Recommendation: member.

\subsection{HD 192424}

Described by Abt (1985) as 'A2 Vp $\lambda$ Boo' and the southern of a 6 arcsec common proper motion pair. This star is listed as a $\lambda$ Boo star in the Renson et al. (1990) catalogue, but Paunzen et al. (2002b) state this star is misclassified. Gerbaldi et al. (2003) found the best fit to the star's spectrum was with solar metallicity. Recommendation: nonmember.

\subsection{HD 192640 (29 Cyg)}

Discovered by Slettebak (1952), this is one of the best studied $\lambda$ Boo stars. It has a spectral type of A0.5 Va- $\lambda$ Boo PHL (Gray 1988). Additional comments there were ' $\lambda 4481$ extremely weak. The $\mathrm{K}$ line and the metallic-line spectrum are intermediate in strength to the A0 and A1 standards. The hydrogen lines have very broad, shallow wings and very shallow, weak cores ... many lines of Fe II are either missing or very weak. The Si II doublet $\lambda \lambda 4128-30$ is also extremely weak. $\delta$ Sct star with an amplitude of $0.03 \mathrm{~V}$ '. We reclassified the star for this work, as 'kA1.5hA7mA0.5 $\lambda$ Boo'. Doubly periodic (Paunzen \& Handler 1996). Well below the line of normal stars (and therefore among the $\lambda$ Boo stars) in the $a$ vs. $b-y$ plot of Maitzen \& Pavlovski (1989b). Meets all the UV criteria for membership (Faraggiana et al. 1990). One of only six stars with the certain member designation in the Renson et al. (1990) catalogue. PHL confirmed in Iliev \& Barzova (1993a), where a strong Ca II K line was noted. Stürenburg (1993) conducted an abundance analysis that showed metals are 1.0-1.5 dex below solar and $[\mathrm{C} / \mathrm{H}]$ $\sim-0.1$. A later abundance analysis shows $\mathrm{C}, \mathrm{N}, \mathrm{O}$, and $\mathrm{S}$ are approximately solar (within 1 or $2 \sigma$ ) and almost all metals studied are 1-2 dex below solar (Heiter et al. 1998). An NLTE abundance analysis of [N], [O], and [S] yielded -0.55 , $-0.15,-0.39$ (Kamp et al. 2001). The low $v \sin i\left(35 \mathrm{~km} \mathrm{~s}^{-1}\right.$, Abt \& Morrell 1995) favours abundance analyses, though interestingly Paunzen et al. (2003) found a higher $v \sin i$ of $80 \mathrm{~km} \mathrm{~s}^{-1}$ when looking in the IR spectrum of this star, and found evidence for interstellar gas and ambiguous evidence for circumstellar dust. Iliev \& Barzova (1998) observed $\mathrm{H} \gamma$ emission which they describe as 'a shell seen nearly 
pole-on', but Paunzen et al. (2003) ruled out an active accretion disk. Key physical parameters are available (Iliev \& Barzova 1995). Recommendation: member.

\subsection{HD 193063}

Noted as a $\lambda$ Boo star in a wide binary with spectral type A0 III by Abt (1985). Also in the Renson et al. (1990) catalogue. A double/multiple star with a magnitude difference $\Delta V=0.62 \pm 0.01$ and separation 5.38 arcsec (Nakos, Sinachopoulos \& van Dessel 1995). There was not much evidence for or against $\lambda$ Boo membership in the literature, so we reobserved this target. We give the spectral types for HD 193063A and B as B9 III and B9 IIIa, respectively. The spectra of the two stars are very similar, with the $\mathrm{H} \gamma$ line slightly narrower in B. Recommendation: nonmember.

\subsection{HD 193256}

Spectral type of A2 Va $\lambda$ Boo PHL in Gray (1988). The comments on this object recorded there are extensive, since this star is in a widely separated $(27.5 \mathrm{arcsec})$ double with HD 193281, with the latter being 1.1 mag brighter. Perhaps, the binarity is what led Renson et al. (1990) to record this star's membership in the $\lambda$ Boo group as uncertain. Stürenburg (1993) carried out an abundance analysis of both objects, where for both stars $[\mathrm{C}]$ is normal, $[\mathrm{Na}]$ is +1 dex and most metals are at -0.5 to -1 dex. The pattern is not the same for each metal for each star. Unusually, $\mathrm{Mg}$ is not weak for either star, in contradiction with Gray's (1988) assessment. Key physical parameters are available for both stars (Iliev \& Barzova 1995). Paunzen et al. (2003) looked for gas and dust around these stars, finding only interstellar gas and no evidence of dust. They recorded $v \sin i$ for each star, obtaining 250 and $95 \mathrm{~km} \mathrm{~s}^{-1}$, respectively. Soubiran et al. (2010) listed $T_{\text {eff }}=7860, \log g=3.74$, and $[\mathrm{Fe} / \mathrm{H}]=-0.95$ for HD 193256. We give the spectral type A9 Vn kA2mA2 $\lambda$ Boo to HD 193256 from our new spectrum. Recommendation: member.

\subsection{HD 193281}

See also entry on HD 193256. Classified as 'A3mA2Vb lambda Boo' (Gray \& Garrison 1987). This is one of those $\lambda$ Boo candidates where the chosen luminosity class has a large bearing on the inferred peculiarity. For instance, the spectral types 'A7 Vn kA2mA2' and 'A2 IVn' both describe the spectrum well, but since the latter does not require the star to be peculiar, it is preferred. The spectrum is a good match to the A2 IVn standard ( $\beta$ Ser), with possibly a slightly weak $\lambda 4481$ line, but the rotational broadening causes uncertainty. Given literature abundance analyses that suggest this is a $\lambda$ Boo star, the evidence is in conflict. Recommendation: uncertain member.

\subsection{HD 193322 D}

Renson et al. (1990) designated the membership of this star in the $\lambda$ Boo group as uncertain. Its spectral type there is recorded as $\mathrm{B} 8 \mathrm{~V} \mathrm{Si}$, and there appears to be no independent suggestion that it should be a member of the $\lambda$ Boo group.

Recommendation: non-member.

\subsection{HD 196821}

Classification as a HgMn star led Hauck et al. (1998) to reject this star from the $\lambda$ Boo group, though interestingly the Renson \& Manfroid (2009) catalogue does not list it as such, but rather as B9-. Its spectral type from Abt \& Morrell (1995) is A0 IIIp ( $\lambda$ Boo)s, with $v \sin i=10 \mathrm{~km} \mathrm{~s}^{-1}$. Hauck et al. (1998) also flagged this star as 'SB?', perhaps because of the low $v \sin i$; the radial velocity from the BSC is $-37 \mathrm{~km} \mathrm{~s}^{-1}$. It occurs in Faraggiana et al.'s (2004) list of $\lambda$ Boo stars with composite spectra. The work of Griffin et al. (2012), refuting the composite spectrum hypothesis, includes this star with the spectral type $\mathrm{A} 0 \mathrm{III}^{+} \mathrm{p} \mathrm{kB} 8 \mathrm{~mA} 1$ (CP). They showed this star is not a spectroscopic binary. They note changes in line profiles and depths that are 'unmistakeable', but state the overall envelope of a line does not move, which they interpret as a variable surface abundance rather than an SB2 spectrum. They confirm the spectral peculiarities but not of the $\lambda$ Boo kind. Recommendation: nonmember.

\subsection{HD 198160}

Gray (1988) gives the spectral type as A2 Vann wk4481, with the note 'Forms a close visual binary with HD 198161 [separation 2.4 arcsec]. The very broad, shallow hydrogen line wings seen in this star may be an effect of rapid rotation (cf. GG), but $\lambda 4481$ is extremely weak. Corbally \& Garrison (1980) classify this star as A2 Vn with A3 Vn for HD 198161. Corbally (1984) lists a spectral type for the pair as A2 III and A3 III, but notes that his (underexposed) $67 \AA / \mathrm{mm}$ spectra give a type of $\mathrm{V}$ for each, and that isochrones put the stars near the main sequence. Note the extremely red $b-y$ colour $[=0.108]$ for an A2 star'.

The abundance analysis by Stürenburg (1993) shows solar $\mathrm{C}, \mathrm{Na}$, and $\mathrm{Mg}$, and moderate underabunances $(-0.5$ to -1 dex) of most metals for both stars, under the assumption that they are twins (with the same $T_{\text {eff }}$ and $\log g$ ), though Gerbaldi et al. (2003) later argued this assumption to be invalid based on magnitude differences of 0.35 and 0.39 in $V$ and $B$, respectively. Iliev \& Barzova (1995) provided key physical parameters (for HD 198160 only). Circumstellar absorption in the Ca II $\mathrm{K}$ line was reported as 'probable' by Holweger \& Rentzsch-Holm (1995). NLTE abundances of [C] and [O] are -0.16 and -0.18 , respectively (Paunzen et al. 1999b), but these are quoted for HD 198160/1, i.e. not as separate entities. Evidence for dust causing an infrared excess was presented 
by Paunzen et al. (2003), where uncertain circumstellar gas absorption was also noted.

The observation of substantial metal deficiency for each star is in favour of membership, but solar NLTE abundances of volatile elements are not confirmed for each individual star. The abundances are suggestive of the typical $\lambda$ Boo abundance pattern, so we tentatively accept both stars as members. Recommendation: member.

\subsection{HD 198161}

See entry on HD 198160. Recommendation: member.

\subsection{HD 200841}

This star was first 'estimated' to be a $\lambda$ Boo star by Olsen (1980), and was subsequently shown to lie well below the line of normal stars in the Maitzen \& Pavlovski (1989b) $\Delta a$ photometry study. However, it was classified by Abt (1984b) as 'A0 Vn', which led Renson et al. (1990) to call it an uncertain $\lambda$ Boo star in their catalogue. We have obtained a new spectrum of this star which we classify as A0 IIIIV(n). The spectrum is a good match to the A0 IVn standard, HR 8451, with the exception of slightly narrower $\mathrm{H}$ lines in HD 200841. Recommendation: non-member.

\subsection{HD 201019}

This star's late spectral type [F3w (for metal weak), Houk $\&$ Cowley 1975], and red colour $(B-V=0.34)$, make it an uncertain member of the $\lambda$ Boo group, as reflected in the Renson et al. (1990) catalogue.

Gray (1988) commented on this star, that its membership as a horizontal branch star seems to be ruled out by low space velocities and by a $\delta c_{1}(=0.21)$ index in common with main-sequence stars. He suggested the late nature of this star might indicate it is evolved, but that it 'lies on the extension of the ' $\lambda$ Bootis distribution' in the $b-y, m_{1}$ plane' (see figure 2, there). He recommended a high-resolution spectrum for further classification. King (1994) recorded a 'doubtful' detection of an IRAS infrared excess. We have reobserved this star and give the spectral type F2 V kA7mA6 $\lambda$ Boo?, where an abundance analysis of volatile elements is required to distinguish between a true $\lambda$ Boo or a Pop. II star. Recommendation: probable member.

\subsection{HD 201184}

The identification of this star as a $\lambda$ Boo member is ambiguous according to Andrievsky et al. (2002). Their spectroscopic analysis provided $T_{\text {eff }}=9970 \mathrm{~K}$ and $\log g=4.2$, which agrees with the spectral type of A0 V (Houk \& SmithMoore 1988) and $B-V=0.01$. Andrievsky et al. (2002) recorded $v \sin i=200 \mathrm{~km} \mathrm{~s}^{-1}$ and found only mild metal underabundances (e.g. $[\mathrm{Fe} / \mathrm{H}]=-0.41$ ) but gave no $\mathrm{C}$ or $\mathrm{N}$ abundances. When investigating the observational properties of $\lambda$ Boo stars, Paunzen et al. (2002a) even used HD 201184 as a normal comparison star, presumably because they were unaware of its slight metal weakness. $u v b y \beta$ observations (Hauck \& Mermilliod 1998) suggest the strongest of hydrogen lines (i.e. around $\mathrm{A} 2 \mathrm{~V}$ ), but the $m_{1}$ and $c_{1}$ indices are consistent with an $\mathrm{A} 0 \mathrm{~V}$ star, and thus point to a mild metal weakness. Cross-referencing the Eggleton \& Tokovinin (2008) catalogue of multiplicity among bright stars shows this is a single star.

In the absence of firm evidence of this being a $\lambda$ Boo star, we reobserved and classified its spectrum. It has the spectral type A0.5 $\mathrm{Vn}$, and is therefore not a $\lambda$ Boo star. Recommendation: non-member.

\subsection{HD 204041}

Classified as 'A1 Vb $\lambda$ Boo PHL' (Gray 1988), with the following comment: 'The metallic-line spectrum and the $\mathrm{K}$ line are similar to the A1 standards. $\lambda 4481$ is weak for that spectral type. The hydrogen-line profiles are peculiar with broad wings but weak cores'. Strömgren colours and an LTE abundance analysis were given in Stürenburg (1993), where normal $\mathrm{C}$ and $\mathrm{Na}$ are shown alongside $\sim 1$ dex underabundances of most metals. A later NLTE abundance analysis (Paunzen et al. 1999b) for $\mathrm{C}$ and $\mathrm{O}$ revealed $[\mathrm{C} / \mathrm{H}]=-0.81$ and $[\mathrm{O} / \mathrm{H}]=-0.38$, and for $\mathrm{N}$ and $\mathrm{S}$ (Kamp et al. 2001) revealed $[\mathrm{N} / \mathrm{H}]=-0.35$ and $[\mathrm{S} / \mathrm{H}]=-0.17$. Key physical parameters are available (Iliev \& Barzova 1995). Gerbaldi et al. (2003) remarked an 'inconsistent UV flux' (see their figure 9). HD 204041 was also included in a study of $\lambda$ Boo stars compared with dusty, normal A stars by Holweger \& Rentzsch-Holm (1995), where its $v \sin i$ is listed as $68 \mathrm{~km} \mathrm{~s}^{-1}$. They reported no evidence of circumstellar material in the Ca II K line core. Solano et al. (2001) remarked this star shows no sign of a companion in the spectrum, and provided an abundance analysis, yet Faraggiana et al. (2004) suspected HD 204041 is a double star.

The abundances do not distinguish unambiguously in favour of $\lambda$ Boo classification, but do hint at it. Recommendation: member.

\subsection{HD 204754}

A known variable star, with a spectral type of B8 III (Hube 1970), and an uncertain member of the $\lambda$ Boo class according to Renson et al. (1990). $T_{\text {eff }}$ and $\log g$ have been recorded in Cenarro et al. (2007) as $12923 \mathrm{~K}$ and 3.50, respectively, making this a very hot $\lambda$ Boo candidate. Cayrel de Strobel et al. (1997) listed $[\mathrm{Fe} / \mathrm{H}]$ as -0.28 , which is not non-solar to any high significance. We obtained a new spectrum for this star, which we classify as B5 III-IVs. We note that the $\mathrm{Ca}$ II $\mathrm{K}$ line is quite strong, probably indicating a substantial interstellar component, and that $\mathrm{Si}$ II and some Ti II lines are enhanced. It is not a $\lambda$ Boo. Recommendation: non-member. 


\subsection{HD 204965}

In the Renson et al. (1990) catalogue as a misclassified member, probably because Hauck (1986) rejected it as a $\lambda$ Boo member for having a high luminosity class (III). Abt $\&$ Morrell (1995) then classified the star as 'A2 Vp Mg II 4481-wk'. It was listed as a spectroscopic binary by Gerbaldi et al. (2003), but this is not recorded on SIMBAD. $u v b y \beta$ photometry (Hauck \& Mermilliod 1998) puts the star near A3, for which the $m_{1}$ value indicates metal weakness but the $c_{1}$ value is high, implying an evolved star or perhaps a binary. There is little evidence in favour of membership, and a new spectrum obtained for this work has spectral type A2 IV 4481-wk; it is not a $\lambda$ Boo star. Recommendation: non-member.

\subsection{HD 207978}

Listed in the Renson et al. (1990) catalogue as a misclassified member. We found no source that recommended membership. This star has a late spectral type (F6IV-V w var, Barry 1970) for a $\lambda$ Boo star, though some spectral types are earlier with no indication of metal weakness (F2 V, Abt \& Morrell 1995). It appears that only the metal-weak classification would have led to a suggestion of this star as a member in the first place. Recommendation: non-member.

\subsection{HD 210111}

Gray (1988) gave the spectral type 'A2hA7mA2 Vas $\lambda$ Boo ... PHL', and the comment: 'hydrogen lines show very weak cores like those of an A7 or possibly F0 star, but broad and very shallow wings. The $\mathrm{K}$ line and the metallic-line spectrum are similar to the A2 standards, with $\lambda 4481$ weak with respect to that type. The spectrum of this star is very similar to that of HD 161817, a well-known blue horizontal-branch star, except for the very broad wings'. The abundance analysis by Stürenburg (1993) showed -1 dex metals, while NLTE abundances (Kamp et al. 2001) for [C] and [O] were -0.45 and -0.20 , respectively. Key physical parameters are available (Iliev \& Barzova 1995). Solano et al. (2001) confirmed the low metal abundances. Known SB, and the subject of a dedicated paper (Paunzen et al. 2012). Recommendation: member.

\subsection{HD 210418}

Andrillat et al. (1995) studied this star's infrared spectrum and remarked that it appears to be a normal star. Gray \& Garrison (1987) gave the spectral type A2mA1 IV-V with the note 'SB2, and therefore the spectrum may be composite and not actually metal weak'. High proper motion, with a transverse velocity of $38.1 \mathrm{~km} \mathrm{~s}^{-1}$. We reject the star due to lack of evidence in favour of membership, but UV observations and an abundance analysis could rule out membership definitively. Recommendation: non-member.

\subsection{HD 212061}

A binary star and one of the first stars identified as a $\lambda$ Boo star (Parenago 1958), but there is no trace of the original report of an abnormal spectrum. One of the few stars in the Maitzen $\&$ Pavlovski (1989b) $\Delta a$ photometry study that lies with the normal stars rather than the $\lambda$ Boo stars. It shows none of the UV characteristics of $\lambda$ Boo stars (Faraggiana et al. 1990), and was described as 'misclassified' in the Renson et al. (1990) catalogue. Indeed, Gray \& Garrison (1987) gave the normal spectral type 'B9.5 III-IV'. Recommendation: nonmember.

\subsection{HD 212150}

Classified as 'A0 Vp $\lambda$ Boo' by Abt \& Morrell (1995), but this is not supported elsewhere. Gerbaldi et al. (2003) reported an inconsistent UV flux and the star is in the list compiled by Faraggiana et al. (2004) of stars that likely display composite spectra, but with no specific comment. Not much is known about this star, and there is no compelling evidence to admit membership at all. Our spectrum of HD 212150, with spectral type B9 IIInp kA0, shows that this is a peculiar star, but it is not a $\lambda$ Boo star. $\lambda 4481$ is normal. Recommendation: non-member.

\subsection{HD 213669}

HD 213669 was given the spectral type kA1hF0mA1 V $\lambda$ Boo by Paunzen et al. (2002b), who noted that bona fide status was pending a full abundance analysis. Atmospheric parameters are also given there, but it is the only paper reporting on this star's $\lambda$ Boo status. We therefore obtained a new spectrum, for which our spectral type is F0.5 V kA2.5mA2.5 $\lambda$ Boo. Abundances would still be nice. No IUE spectrum is available. Recommendation: probable member.

\subsection{HD 214454}

Abt \& Morrell (1995) gave the spectral type 'F0 Vp ( $\lambda$ Boo; metals A6)'. SB according to Gerbaldi et al. (2003), but most probable configuration is single star according to the Eggleton \& Tokovinin (2008) catalogue of multiplicity among bright stars. An abundance analysis (Erspamer \& North 2003) showed a mild $\lambda$ Boo pattern. A new spectrum obtained for this work has the spectral type A9.5 V kA7mA6. There is some mild metal weakness but $\lambda 4481$ is normal-this is not a $\lambda$ Boo star. Recommendation: non-member.

\subsection{HD 216847}

Spectral type hF0mA3 Vn $\lambda$ Boo (Paunzen et al. 2001), with a comment in the third paper of that series: 'The hydrogen lines agree very well with F0, although it is a very rapid rotator, it is clearly metal-weak' (Paunzen 2001). Inconsistent UV flux and suspected binary (Gerbaldi et al. 2003). We indicate 
only probable membership until a spectrum can be classified against rapidly rotating standards to ensure it is of the $\lambda$ Boo type. Recommendation: probable member.

\subsection{HD 217782 (2 And)}

Identified by Parenago (1958) as a $\lambda$ Boo star because it was an early A star below the main sequence, but no metal weakness was actually reported (Sargent 1965). Falls on the straight line representing normal unreddened stars in the $\Delta a$ photometry study of Maitzen \& Pavlovski (1989b). Abt \& Morrell (1995) gave the classification A1 V. Faraggiana et al. (1990) found none of the UV criteria for $\lambda$ Boo membership are met, but the star does have the required negative $\Delta m_{1}$ index. There is disagreement in the literature over whether this is a $\lambda$ Boo star, with at least two references arguing on each side. This is probably why its membership is uncertain in the Renson et al. (1990) catalogue. Described as 'LB, shell' in the IR study of Andrillat et al. (1995, see additional comments, end Section 4, there). Evidence of Na I D lines and circumstellar Ca II K line absorption (Hauck et al. 1998). Appears in the list of Faraggiana et al. (2004) of stars with composite spectra, following the comment "composite, Hipparcos' in Gerbaldi et al. (2003); the WDS catalogue (Mason et al. 2001) lists a companion at $0.2 \operatorname{arcsec}$ that is $2.5 \mathrm{mag}$ fainter. We have obtained a new spectrum and classified it on the MK system as A2 IIIn. Recommendation: non-member.

\subsection{HD 218396 (HR 8799)}

Identified as a weak-lined star by Abt \& Morrell (1995). One of the few stars Gerbaldi et al. (2003) did not call a composite, with a spectral type listed there as kA5hF0mA5 V $\lambda$ Boo. HR 8799 is known to host four planets (e.g. Soummer et al. 2011). It is also a $\gamma$ Dor star, and a whole paper linking the $\lambda$ Boo and $\gamma$ Dor classes focussed on this object (Gray \& Kaye 1999; see also Moya et al. 2010, Wright et al. 2011, and Sódor et al. 2014 for asteroseimic studies of this star). The reader is referred to Gray \& Kaye (1999) for more details. Recommendation: member.

\subsection{HD 220061}

Listed in the Renson et al. (1990) catalogue, and also called $\lambda$ Boo by Abt \& Morrell (1995). This was called a 'normal star' of spectral type A5 V in the IR study of Andrillat et al. (1995). The spectral type in Gerbaldi et al. (2003) is A8 V kA5mA5 $\lambda 4481$-weak (probably taken from Gray et al. 2001), and it is noted there as an RV variable, but Griffin et al. (2012) found no line-profile variations to support binarity and played down those 'RV variations'. They reported only mild metal deficiencies of $<0.5 \mathrm{dex}$, and described the $\lambda$ Boo classification of this star as 'questionable' and 'tentative'. A cursory inspection of the UV spectrum (this work) was inconclusive on the $\lambda$ Boo nature, possibly because of the late hydrogen line type. At $v \sin i=150 \mathrm{~km} \mathrm{~s}^{-1}$ (Bernacca
$\&$ Perinotto 1970), an abundance analysis may be difficult. This star could be considered as an extremely mild $\lambda$ Boo star, but the evidence is clearly discordant. Recommendation: uncertain member.

\subsection{HD 220278}

Its spectral type in Vol. IV of the Michigan Catalogue (Houk \& Smith-Moore 1988) is A3 V, and Cowley et al. (1969) gave A5 Vn. Its spectrum is considered composite (e.g. Faraggiana et al. 2004) because it is separated by less than 2 arcsec from a star less than 2.2 mag fainter. Actual values of the separation and magnitude difference from the WDS catalogue are 0.2 arcsec and $1.1 \mathrm{mag}$ (Mason et al. 2001). HD 220278 does appear in the catalogue of $\lambda$ Boo stars by Renson et al. (1990), and is given as ' $\lambda$ Boo, CP(Mg wk), close binary' in Ohanesyan (2008), where the EW of the $\lambda \lambda 2786-2810$ and 4481 features are described for many stars, but its status as a true $\lambda$ Boo star is clearly doubtful. Recommendation: uncertain member.

\subsection{HD 221756}

Spectral type of 'A1 Va ${ }^{+}$( $\lambda$ Boo) ... P/NHL' in Gray (1988), with the note: 'The $\mathrm{K}$ line and the metallic-line spectrum are similar to the A1 standards, except that $\lambda 4481$ appears weak. The hydrogen lines show very broad wings with slightly weak cores. This star may be a transitional type between type NHL and type PHL. This star is a suspected photometric variable'. Reobservation for this work the star for this work yielded the spectral type 'A3 $\mathrm{Va}^{+} \mathrm{kA} 1 \mathrm{~mA} 0.5$ ( $\lambda$ Boo)'. LTE abundance analysis (Stürenburg 1993) is incomplete and shows only mild metal deficiency $(\sim 0.5 \mathrm{dex})$, but it does have $\lambda$ Boo features in the infrared (Andrillat et al. 1995), and has a circumstellar Na I D line. Key physical parameters are available (Iliev \& Barzova 1995). NLTE abundances from Kamp et al. (2001) show solar $\mathrm{O}$ and $\mathrm{S} ;[\mathrm{N} / \mathrm{H}]=-0.5$. Paunzen et al. (1999a) included this star in their paper on 'Accurate LTE abundances of seven well-established $\lambda$ Bootis stars', finding $[\mathrm{C} / \mathrm{H}]=0.0$ and metal abundances on the order -0.5 dex. Paunzen et al. (2003) reported both dust and circumstellar gas. The $60-\mu \mathrm{m}$ flux measurement is perhaps the most excessive they measured (their figure 1). Gerbaldi et al. (2003) noted RV variability, though Griffin et al. (2012) noted its $\mathrm{RV}$ to be constant to well within their obtained precision, and found no evidence for an SB2 spectrum-a conclusion that they say was also reached by Stütz \& Paunzen (2006). Recommendation: member.

\subsection{HD 222303}

Maitzen \& Pavlovski (1989b) complained of unknown reddening for this star (whose $b-y$ value is 0.414 !), but commented it would still be in the $\lambda$ Boo regime in their $a$ vs. $b-y$ plot. It was perhaps the high $b-y$ that led Paunzen et al. (1997) to reject this star as a $\lambda$ Boo member. It does appear in 
the Renson et al. (1990) catalogue, and its spectral type from Abt (1984b) is 'A6VmA1 $\lambda$ Boo?'. The metal weakness is thus evident and a red colour is not quite sufficient ground for exclusion on its own. We obtained a new spectrum of this star, which is certainly peculiar but not typical for a $\lambda$ Boo star. The spectrum does not match at any type. We tentatively give the rough spectral type A9 III (met wk A3), but 'metal weak, late-A giant' is as precise as it is possible to be. Because of the peculiar spectrum, we cannot definitively rule out this star as a member of the $\lambda$ Boo class. Further study is recommended. Recommendation: uncertain member.

\subsection{HD 223352}

Abt \& Morrell (1995) classified as A0 Vp (Lam Boo)n. This is a 2.4-d eclipsing binary (Shobbrook 2005) within the Blanco 1 cluster. Inspection of the published light curve, at its somewhat limited resolution, indicates the secondary contributes on the order 10-20 per cent of the combined light output, and its lines may be visible in a spectrum of good signal to noise. Strömgren photometry $(\beta=2.893$, $b-y=-0.004, m_{1}=0.153, c_{1}=1.019$; Hauck \& Mermilliod 1998) would suggest a slightly metal weak star around A2. In this work, we re-examined the spectrum and gave the spectral type A0 Van kB9 ((Lam Boo)), with the comment ' $\lambda 4481$ is weak. He I and hydrogen line profiles put spectral type firmly at A0, but $\mathrm{K}$ line and metallic-line spectrum generally weak and closer to B9. A very mild (marginal) Lambda Boo'. Taking both the spectroscopic and binary information into account, we consider this a probable member of the $\lambda$ Boo group. Recommendation: probable member.

\subsection{HD 225180}

A1 IVp $\lambda$ Boo according to Abt \& Morrell (1995), but a few literature spectral types suggesting an early-A giant exist, including A1 II-III by Gray \& Garrison (1987). Hauck et al. (1998) recommended this star be excluded from the $\lambda$ Boo group. Its $\mathrm{Na}$ I doublet warranted special mention and a figure (their figure 4), and they wrote that 'these lines are strong and all the lines of the optical spectrum possess a shell component. The Na I doublet presents a double structure in which a photospheric and a redshift shell component may be identified. The shell component has an asymmetric background' Recommendation: non-member.

\subsection{HD 225218}

Another Abt $\&$ Morrell (1995) $\lambda$ Boo star in a double or multiple system, having 'duplicity induced variability' (Gerbaldi et al. 2003). The star is a slow rotator, probably as a result of the binarity. The B9 III spectral type from Cowley et al. (1969) does not agree with the A3 V classifications from Barry (1970) and Abt \& Morrell (1995). No strong evidence for $\lambda$ Boo membership. We obtained two new spectra of this star, and in neither does it look like a $\lambda$ Boo star. The $\mathrm{H}$ lines could be placed at either A3 IV or A5 IV, but the stronger cores argue slightly for the former. Both the $\mathrm{Ca}$ II $\mathrm{K}$ line and $\mathrm{Ca}$ I 4226 are weak compared to other metals, and lines of Sr II are slightly strong, so this could be a mild Am star. The compromise type that describes both spectra satisfactorily is A4 IV. The WDS catalogue (Mason et al. 2001) lists a separation of 0.1 arcsec for this star, with a note that it is a spectroscopic binary, which might explain the difficulty in classifying the spectrum. Recommendation: non-member.

\subsection{HD 228509}

The literature on this star is scarce. The $u v b y \beta$ photometry from Hauck \& Mermilliod (1998) indicates a late-A temperature type with metal line strength near A0. We obtained a new spectrum of this star and classify it as A9 V(n) kA5mA5, with a slight weakness in the $\lambda 4481$ line. Whether or not the $\lambda 4481$ line is weak enough to warrant $\lambda$ Boo membership is uncertain, therefore a high-resolution spectrum and full abundance analysis, including volatile elements, will be the discriminant. The high $v \sin i$ may prove obstructive in this regard. Recommendation: uncertain member.

\subsection{HD 245185}

Gray \& Corbally (1998) observed this star but did not note any $\lambda$ Boo peculiarities, instead they classified it as 'A3 Vae $\mathrm{Bd}<\mathrm{Nem1}$ '. Folsom et al. (2012) wrote: 'We find $\mathrm{He}, \mathrm{C}, \mathrm{N}$, and $\mathrm{O}$ abundances that are consistent with solar, and iron peak abundances that are $\sim 0.8$ dex below solar. While the uncertainties are relatively large for this star, the strong iron peak underabundances indicate $\lambda$ Boo peculiarities'. Their analysis showed that metals are $\sim 2 \sigma$ below solar. As such a young star, it is probably a spectrum variable. Recommendation: uncertain member.

\subsection{HD 261904 (NGC 2264-138)}

Gray \& Corbally (1998) gave the spectral type A0 Va $((\lambda$ Boo $))$ with the comment that the star has a slightly weak Mg II 4481 line and other slightly weak metal lines. Paunzen's (2001) list of 'new and confirmed' $\lambda$ Boo stars gives the spectral type as A0.5 V $\lambda$ Boo. Andrievsky et al. (2002) determined the $\mathrm{Mg}$ and $\mathrm{Fe}$ abundances to be supersolar, and thus stated this star 'can be definitely ruled out' as a member of the $\lambda$ Boo group. Inspection of the classification resolution spectrum in Paunzen's (2000) PhD thesis indicates a star that is earlier than $\mathrm{A} 0.5 \mathrm{~V}$, in that the hydrogen cores are too shallow and helium lines too strong for this spectral type. Recommendation: uncertain member.

\subsection{HD 278937}

Gray \& Corbally (1998) observed this star (IP Per) on two separate occasions, two years apart, and found the metal weakness to change in severity. They classified it as 
kA3hA7mA4 III:er, where the uncertainty applies to the luminosity class and the emission features are redshifted compared to the photospheric $\mathrm{H}$ line cores. They described it as 'slightly metal-weak, even though it does not show all of the characteristics of a $\lambda$ Boo star'. Folsom et al. (2012) carried out an abundance analysis, and wrote: 'We find solar abundances for $\mathrm{C}, \mathrm{N}, \mathrm{O}$ and $\mathrm{S}$, while iron peak elements as well as $\mathrm{Na}, \mathrm{Mg}$ and $\mathrm{Si}$ are between 0.5 and 0.7 dex underabundant. This star shows clear $\lambda$ Boo peculiarities'. Recommendation: probable member.

\subsection{HD 290492}

Paunzen \& Gray (1997) recorded this star as A0.5 Vb ( $\lambda$ Boo) with the comment 'Mild $\lambda$ Bootis character, star belongs to the Orion OB1 belt (Guetter 1981), classified therein as A0 V. Close binary system $(\Delta m=0.9, d=2$ arcsec $)$ which was resolved without any contamination'. Marchetti et al. (2001), on the other hand, said of HD 290492 that it is a close binary 'with values of the separation and of the magnitude difference such that only a composite spectrum can be observed'. and directly contested the Paunzen \& Gray (1997) result (see their section 5). According to the WDS catalogue (Mason et al. 2001), the separation is 0.7 arcsec and the magnitude difference is $0.6 \mathrm{mag}$. When we obtained a spectrum the seeing was poor, and we did not see this as a binary. Our classification is $\mathrm{A} 1 \mathrm{~V}(\mathrm{n}) \mathrm{kA} 0.5 \mathrm{~mA} 0.5$, and we note that the spectrum is generally slightly metal weak; Mg II 4481 is not extra weak. Clearly, for this system it will be necessary to obtain resolved high-resolution spectra and carry out a full abundance analysis of each star, including for volatile elements, before a firm membership evaluation can take place. Recommendation: uncertain member.

\subsection{HD 290799}

Identified as a young $\lambda$ Boo star by Gray \& Corbally (1993), where it is listed as $\mathrm{A} 2 \mathrm{Vb} \lambda$ Boo PHL. Described as one of the true ZAMS stars of that survey of OB associations for $\lambda$ Boo stars. [paraphrasing:] The hydrogen line core type is A6-A7, the metallic line type is A2 and the $\mathrm{Ca}$ II $\mathrm{K}$ line type is slightly earlier. $\mathrm{Mg}$ II 4481 is slightly earlier than the metallic-line spectrum. 'The $\left[m_{1}\right]$ index confirms the metal weak character of the star. The $\beta$ index is too small for an early-A-type dwarf, another typical signature of a PHL $\lambda$ Bootis star'. Key physical parameters are available (Iliev \& Barzova 1995). An abundance analysis by Andrievsky et al. (2002) confirmed this star's membership in the $\lambda$ Boo class, with normal $\mathrm{C}$ and $\mathrm{O}$, and $[Z]=-1.0$ dex. The NLTE sodium abundance was determined there, from the $D_{1}$ and $D_{2}$ doublet, to be +0.45 dex. Additionally, we obtained a new spectrum, which we classify as A7 V kA2mA2 $\lambda$ Boo, with the comment: PHL, cores match A7, but the wings are closer to A2. The metal line morphology matches the A7 type, but at a strength consistent with A2. The $\lambda 4481$ line is substantially weakened. Recommendation: member.

\subsection{HD 294253}

Spectral type of B9.5 Va ( $\lambda$ Boo) in Paunzen \& Gray (1997), with the comment that this is one of the hottest as well as youngest $\lambda$ Boo stars. We reobserved this star at 3.6$\AA$ resolution for this work, and assigned the spectral type 'A0 Va kB8.5 ( $\lambda$ Boo)'. Andrievsky et al. (2002) conducted an abundance analysis, but the results were ambiguous as to the star's $\lambda$ Boo nature, in that although $\mathrm{O}$ was solar, metals were only slightly subsolar, and only a few metals could have their abundances measured. Recommendation: member.

\subsection{TYC 3680-215-1 (BSD 8-403)}

A $\lambda$ Boo member on Gray's website that was unpublished until now. We reobserved the star for this work. Its spectrum is consistent with an extreme $\lambda$ Boo star with a hydrogen line type of $\mathrm{A} 0 \mathrm{Va}^{-}$. No metallic lines are visible, with the exception of a narrow $\mathrm{Ca}$ II $\mathrm{K}$ line, which is probably interstellar. Diffuse interstellar band at $\lambda 4420$ is visible, again suggesting significant interstellar absorption. This extreme metallic-line weakness makes it impossible to assign a metallic-line class. We thus admit BSD8-403 to the $\lambda$ Boo class. Note that the $B$ and $V$ magnitudes on SIMBAD are incorrect; magnitudes in $V, B-V$ and $U-B$ are $11.10,0.29$ and 0.14 , respectively (Mermilliod, Mermilliod, \& Hauck 1997). Recommendation: member.

\subsection{TYC 4774-866-1 (T Ori)}

Gray \& Corbally (1998) looked at T Ori and did not note any $\lambda$ Boo peculiarity, even though they were looking for young $\lambda$ Boo stars. They classified it as 'A3 IVeb Bd $>\mathrm{Npc1}$ ', that is, an emission line star with a slightly blueshifted component with respect to $\mathrm{H}$ line cores, having a moderately strong Balmer decrement, and displaying a P Cygni profile. Folsom et al. (2012) concluded they could see $\lambda$ Boo peculiarities, and wrote: 'The abundances for $\mathrm{He}, \mathrm{C}, \mathrm{N}$ and $\mathrm{O}$ are all consistent with solar, while the $\mathrm{S}$ abundance is almost $3 \sigma$ above solar... The iron peak abundances are clearly $\sim 0.5$ dex below solar. We conclude that $\mathrm{T}$ Ori has clear $\lambda$ Boo peculiarities'. We were therefore motivated to reobserve this target. From our spectrum, we assign the spectral type A8 Ve kA2mA2 Bd> Npc1. The $\lambda 4481$ line is normal for an A8 star. This is almost certainly a spectrum variable; in our particular spectrum it did not appear to be a $\lambda$ Boo star, but at some times it may have a $\lambda$ Boo nature. Recommendation: uncertain member.

\section{TABLE AND CONCLUSIONS}

The information in the extended table (Table 1) in this section is a compilation of literature values alongside values we provide ourselves. The SIMBAD identifier, $V$ and $B-V$ values are taken directly from the SIMBAD data base. Upon noticing some inaccuracies in the SIMBAD $V$ and $B-V$ 
Table 1. Summary table for the stars in Section 2. Column 3 indicates the membership recommendation given in Section 2, with symbols: $\checkmark=$ member; $\circ=$ probable member; ? = uncertain member; $x=$ non-member. Column 4 the transverse velocity in km s ${ }^{-1}$. Columns 5 and 6 are Johnson $V$ and $B-V$ magnitudes. Column 7 contains spectral types as specified in the text. Column 8 gives the projected stellar rotational velocity in $\mathrm{km} \mathrm{s}^{-1}$, for which references are given in Table 2.

\begin{tabular}{|c|c|c|c|c|c|c|c|}
\hline HD number & SIMBAD identifier & Member? & T. Vel. & $V$ & $B-V$ & Sp.T. & $v \sin i$ \\
\hline HD 3 & HR 1 & $?$ & 21.9 & 6.71 & 0.06 & A0 Vn $(\lambda$ Boo $)$ & $228^{a}$ \\
\hline HD 319 & HR 12 & $\checkmark$ & 28.9 & 5.94 & 0.13 & kA1hA7mA2 Vb $\lambda$ Boo & $59^{a}$ \\
\hline HD 2904 & HR 129 & $?$ & 25.6 & 6.38 & -0.05 & A0 Vnn $(\lambda$ Boo $)$ & $243^{a}$ \\
\hline HD 4158 & HD 4158 & $\checkmark$ & & 9.54 & 0.22 & F3 V m-3 & \\
\hline HD 5789 & HR 283 & $x$ & 15.6 & 6.06 & -0.03 & B9.5 Vnn $(\lambda$ Boo $)$ & $300^{b}$ \\
\hline HD 6173 & HD 6173 & ? & & 8.52 & 0.13 & A0 IIIn? & \\
\hline HD 6870 & $\mathrm{~V} *$ BS Tuc & $\checkmark$ & 59.9 & 7.45 & 0.26 & $\mathrm{kA} 2 \mathrm{hA} 7 \mathrm{~mA} 2 \lambda$ Boo & $130^{s}$ \\
\hline HD 7908 & HD 7908 & $\checkmark$ & 13.9 & 7.32 & 0.24 & $\mathrm{hF} 0 \mathrm{~mA} 3 \lambda$ Boo & \\
\hline HD 9100 & $\mathrm{~V} * \mathrm{VX}$ Psc & $x$ & 40.5 & 6.02 & 0.14 & $\mathrm{~A} 3 \mathrm{Vb} \lambda$ Boo & $123^{a}$ \\
\hline HD 11413 & $\mathrm{~V} * \mathrm{BD}$ Phe & $\checkmark$ & 17.7 & 5.94 & 0.14 & kA0.5hF0mA0.5 Va $\lambda$ Boo & $139^{a}$ \\
\hline HD 11502 & HR 545 & $x$ & & 4.70 & -0.14 & A0 IV-V(n) kB8 ( $\lambda$ Boo) & $179^{b}$ \\
\hline HD 11503 & $\mathrm{~V} *$ gam Ari & $x$ & 29.6 & 4.52 & -0.03 & knA0hA3 (IV) SiSr & $201^{c}$ \\
\hline HD 11905 & HR 562 & $x$ & 11.0 & 6.77 & -0.07 & B9 HgMn & $68^{d}$ \\
\hline HD 13755 & $\mathrm{~V} * \mathrm{CV}$ Phe & $\checkmark$ & 10.6 & 7.83 & 0.28 & $\mathrm{hF} 2 \mathrm{~mA} 5 \mathrm{~V} \lambda$ Boo & \\
\hline HD 15164 & HD 15164 & $\checkmark$ & 23.2 & 8.27 & 0.31 & $\mathrm{~F} 1 \mathrm{~V}$ kA7mA6 $(\lambda$ Boo $) ?$ & \\
\hline HD 15165 A & $\mathrm{V} * \mathrm{VW}$ Ari & $\checkmark$ & 23.2 & 6.69 & 0.25 & $\mathrm{~F} 2 \mathrm{~V}$ kA2mA2 $\lambda$ Boo? & $129^{a}$ \\
\hline HD 16811 & * 34 Ari & $x$ & 30.5 & 5.74 & -0.03 & A0 IVn & $175^{a}$ \\
\hline HD 16955 & HR 803 & $x$ & 3.9 & 6.36 & 0.10 & A3 V & $175^{a}$ \\
\hline HD 16964 A/B & HD 16964 & $x$ & 7.8 & 8.86 & 0.08 & A0 IV-V; A0.5 IVn & \\
\hline HD 17138 & $\mathrm{~V} * \mathrm{RZ}$ Cas & $\circ$ & 11.5 & 6.26 & 0.14 & $\mathrm{~A} 3 \mathrm{~V}$ & $81^{b}$ \\
\hline HD 21335 & HR 1036 & $x$ & 21.3 & 6.57 & 0.15 & A3 IVn & $217^{a}$ \\
\hline HD 22470 & $\mathrm{~V} *$ EG Eri & $x$ & 24.2 & 5.23 & -0.13 & B9 Si & $75^{c}$ \\
\hline HD 23258 & HR 1137 & $\checkmark$ & 11.1 & 6.08 & 0.03 & kB9.5hA3mB9.5 V ( $\lambda$ Boo $)$ & $123^{a}$ \\
\hline HD 23392 & HD 23392 & $\checkmark$ & 13.1 & 8.24 & 0.05 & $\mathrm{~A} 0 \mathrm{Va}^{-}(\lambda \mathrm{Boo})$ & \\
\hline HD 24712 & $\mathrm{~V} *$ DO Eri & $x$ & 18.6 & 5.99 & 0.30 & A9 $\mathrm{Vp} \mathrm{SrCrEu}$ & $18^{c}$ \\
\hline HD 24472 & HD 24472 & $\circ$ & 18.3 & 7.10 & 0.30 & $*$ & \\
\hline HD 26801 & HD 26801 & $x$ & 4.9 & 7.72 & 0.00 & A0 III $\lambda$ Boo? & \\
\hline HD 27404 & $\mathrm{~V} * \mathrm{~V} 1140 \mathrm{Tau}$ & $x$ & 38.1 & 7.92 & 0.26 & $\mathrm{Ap} \mathrm{Si}$ & $37^{u}$ \\
\hline HD 30422 & $\mathrm{~V} *$ EX Eri & $\checkmark$ & 4.8 & 6.18 & 0.17 & A7 V kA3mA3 ( $\lambda$ Boo $)$ & $128^{a}$ \\
\hline HD 30739 & * 2 Ori & $x$ & 9.8 & 4.35 & 0.02 & A0.5 IVn & $218^{b}$ \\
\hline HD 31293 & HD 31293 & $x$ & 16.4 & 7.06 & 0.12 & A0 Vaer $\mathrm{Bd}<\mathrm{Nem} 5$ & $116^{n}$ \\
\hline HD 31295 & * 7 Ori & $\checkmark$ & 22.9 & 4.66 & 0.08 & $\mathrm{kA} 0 \mathrm{hA} 3 \mathrm{~mA} 0 \mathrm{Va}^{-} \lambda$ Boo & $108^{b}$ \\
\hline HD 34787 & * 16 Cam & $x$ & 28.0 & 5.25 & -0.02 & A0 IIIn & $217^{a}$ \\
\hline HD 34797 & $\mathrm{~V} * \mathrm{TX}$ Lep & $x$ & 12.0 & 6.54 & -0.11 & B7 Vp He-wk & $80^{e}$ \\
\hline HD 35242 & $\mathrm{~V} * \mathrm{~V} 1649$ Ori & $\checkmark$ & 13.1 & 6.34 & 0.12 & $\mathrm{kA} 0.5 \mathrm{hA} 3 \mathrm{~mA} 1 \mathrm{Va} \lambda$ Boo & $86^{a}$ \\
\hline HD 36496 & HR 1853 & $?$ & 7.6 & 6.29 & 0.21 & A5 Vn & $196^{a}$ \\
\hline HD 36726 & HD 36726 & $\checkmark$ & & 8.85 & 0.09 & kA0hA5mA0 V $\lambda$ Boo & $80^{g}$ \\
\hline HD 37411 & HD 37411 & $\checkmark$ & & 9.86 & 0.11 & hA2 Vae kB8mB8 $\lambda$ Boo & \\
\hline HD 37886 & HD 37886 & $x$ & & 9.05 & -0.06 & Ap HgMn & $19^{t}$ \\
\hline HD 38043 & HD 38043 & $\checkmark$ & 5.1 & 9.45 & 0.22 & F1 V kA5mA3 $\lambda$ Boo? & \\
\hline HD 38545 & * 131 Tau & $x$ & 20.6 & 5.73 & 0.07 & $\mathrm{~A} 2 \mathrm{Va}^{+} \lambda$ Boo & $191^{a}$ \\
\hline HD 39283 & * ksi Aur & $x$ & 6.2 & 4.97 & 0.05 & $\mathrm{~A} 1 \mathrm{Va}$ & $68^{b}$ \\
\hline HD 39421 & HR 2039 & $?$ & 15.1 & 5.96 & 0.08 & $\mathrm{~A} 1 \mathrm{Vn}$ & $227^{a}$ \\
\hline HD 40588 & HR 2110 & $\checkmark$ & 6.9 & 6.19 & 0.09 & $\mathrm{~A} 3 \mathrm{~V} \mathrm{kA} 0.5 \mathrm{~mA} 0 \lambda$ Boo & $123^{a}$ \\
\hline HD 41580 & HD 41580 & $x$ & 24.5 & 7.19 & -0.06 & A1 IIIp Si & \\
\hline HD 42503 & $\mathrm{~V} * \mathrm{AU} \mathrm{Col}$ & $\checkmark$ & 13.5 & 7.43 & 0.17 & hA9 Vn kA2mA2 $\lambda$ Boo & \\
\hline HD 47152 & * 53 Aur & $x$ & 11.6 & 5.75 & 0.01 & $\mathrm{~B} 9 \mathrm{Mn}+\mathrm{F} 0 \mathrm{~m}$ & $33^{c}$ \\
\hline HD 54272 & HD 54272 & $?$ & & 8.77 & 0.25 & $\mathrm{kA} 3 \mathrm{hF} 2 \mathrm{~mA} 3 \mathrm{~V} \lambda$ Boo & \\
\hline HD 56405 & HR 2758 & $x$ & 18.3 & 5.45 & 0.09 & A2 Va & $159^{a}$ \\
\hline HD 64491 & $\mathrm{~V} *$ DD Lyn & $\checkmark$ & 17.8 & 6.23 & 0.25 & F1 Vs kA3mA3 $\lambda$ Boo & $23^{c}$ \\
\hline HD 66684 & CCDM J08056+2732AB & $x$ & 17.3 & 6.21 & 0.01 & B9 Va+A1 IVn & $75^{a}$ \\
\hline HD 66920 & HR 3171 & $x$ & 13.1 & 6.33 & 0.14 & A3 V & \\
\hline HD 68695 & HD 68695 & $?$ & 6.6 & 9.87 & 0.06 & $\mathrm{~A} 0 \mathrm{Ve}$ & $44^{n}$ \\
\hline HD 68758 & HR 3230 & $x$ & 30.8 & 6.53 & 0.06 & A1 IVp & $270^{a}$ \\
\hline HD 73210 & HD 73210 & $x$ & 37.1 & 6.75 & 0.16 & A5 IIIs & $80^{b}$ \\
\hline HD 73345 & $\mathrm{~V} * \mathrm{CY} \mathrm{Cnc}$ & $x$ & 31.1 & 8.14 & 0.20 & A7 V kA8 & $98^{b}$ \\
\hline HD 73872 & HD 73872 & $x$ & & 8.34 & 0.21 & kA6hA8mA6 V(n) & $180^{b}$ \\
\hline HD 74873 & $* 50 \mathrm{Cnc}$ & $\checkmark$ & 21.1 & 5.89 & 0.11 & kA0.5hA5mA0.5 V $\lambda$ Boo & $130^{i}$ \\
\hline HD 74911 & HD 74911 & $x$ & 20.4 & 8.51 & 0.13 & A2 IV (4481-wk) & $190^{g}$ \\
\hline
\end{tabular}


Table 1. Continued

\begin{tabular}{|c|c|c|c|c|c|c|c|}
\hline HD number & SIMBAD identifier & Member? & T. Vel. & $V$ & $B-V$ & Sp.T. & $v \sin i$ \\
\hline HD 75654 & $\mathrm{~V} * \mathrm{HZ} \mathrm{Vel}$ & $\checkmark$ & 27.1 & 6.38 & 0.22 & kA3hA7mA3 V $\lambda$ Boo & $45^{c}$ \\
\hline HD 78316 & $\mathrm{~V} *$ kap Cnc & $x$ & 17.9 & 5.24 & -0.09 & B8 IIIp HgMnEu 4481-wk & $18^{c}$ \\
\hline HD 78661 & HR 3635 & $x$ & 14.1 & 6.48 & 0.33 & $\mathrm{hF} 2 \mathrm{~mA} 8 \mathrm{~V}$ & $82^{c}$ \\
\hline HD 79025 & HR 3647 & $x$ & 21.7 & 6.48 & 0.16 & A9 Vn & \\
\hline HD 79108 & HR 3651 & $x$ & 24.3 & 6.14 & 0.00 & $\mathrm{~A} 0 \mathrm{~V} \lambda \mathrm{Boo}$ & $172^{a}$ \\
\hline HD 79469 & LTT 12431 & $x$ & 55.1 & 3.88 & -0.06 & B9.5 IV (CII) & $100^{b}$ \\
\hline HD 80081 & * 38 Lyn & $x$ & 23.5 & 3.82 & 0.06 & $\mathrm{~A} 2 \mathrm{IV}^{-}$ & $160^{b}$ \\
\hline HD 81104 & *21 UMa & $x$ & 40.9 & 7.66 & 0.13 & A3 Van & \\
\hline HD 81290 & HD 81290 & $\circ$ & 18.3 & 8.89 & 0.26 & $\mathrm{~F} 2 \mathrm{~V}$ kA3mA3 $(\lambda$ Boo?) & $56^{h}$ \\
\hline HD 82573 & HR 3796 & $x$ & 19.4 & 5.74 & 0.14 & $\mathrm{~A} 3 \mathrm{~V}$ & $75^{c}$ \\
\hline HD 83041 & $\mathrm{~V} * \mathrm{AK}$ Ant & $\circ$ & 25.3 & 8.79 & 0.31 & $\mathrm{~F} 1 \mathrm{~V}$ kA3mA2 $\lambda$ Boo & $95^{h}$ \\
\hline HD 83277 & HD 83277 & $\circ$ & 43.2 & 8.29 & 0.28 & $\mathrm{~F} 1.5 \mathrm{~V} \mathrm{kA} 3 \mathrm{~mA} 3 \lambda$ Boo? & \\
\hline HD 84123 & HD 84123 & $\checkmark$ & 53.5 & 6.85 & 0.27 & $\mathrm{hF} 2 \mathrm{~V}$ kA6mA6 $\lambda$ Boo & $28^{g}$ \\
\hline HD 84948 & HD 84948 & $\circ$ & 6.7 & 8.12 & 0.30 & F1.5 Vs kA5mA5 $\lambda$ Boo? & $45(\mathrm{~A}) / 55(\mathrm{~B})^{h}$ \\
\hline HD 87271 & V* GM Leo & $\checkmark$ & 4.3 & 7.13 & 0.18 & kA9hA9mA0 V $\lambda$ Boo & \\
\hline HD 87696 & $* 21 \mathrm{LMi}$ & $x$ & 7.1 & 4.49 & 0.18 & $\mathrm{~A} 7 \mathrm{~V}$ & $155^{b}$ \\
\hline HD 89239 & HR 4041 & $x$ & 18.6 & 6.53 & -0.02 & B9.5 V & $149^{a}$ \\
\hline HD 89353 & $\mathrm{~V} * \mathrm{AG}$ Ant & $x$ & 74.7 & 5.53 & 0.23 & B9.5 Ib-II & \\
\hline HD 90821 & HD 90821 & $x$ & & 9.45 & 0.09 & A3 IV-V & $150^{g}$ \\
\hline HD 91130 & * $33 \mathrm{LMi}$ & $\checkmark$ & 7.1 & 5.91 & 0.10 & kA0hA1mA0 Va ( $\lambda$ Boo $)$ & $207^{a}$ \\
\hline HD 97411 & HR 4347 & $x$ & 23.5 & 6.10 & 0.00 & A0 V (4481-wk) & $33^{a}$ \\
\hline HD 97773 & HD 97773 & $?$ & 23.3 & 7.55 & 0.25 & hA8mA3 V $\lambda$ Boo? & \\
\hline HD 97937 & HR 4366 & $?$ & 20.3 & 6.66 & 0.27 & F1 V kA9mA6 & $133^{a}$ \\
\hline HD 98353 & * $55 \mathrm{UMa}$ & $x$ & 24.5 & 4.80 & 0.10 & A1 Va; composite. & $60^{b}$ \\
\hline HD 98772 & HR 4391 & $x$ & 14.7 & 6.03 & 0.08 & $\mathrm{~A} 1 \mathrm{Va}$ & $249^{a}$ \\
\hline HD 100546 & HD 100546 & $?$ & 17.9 & 6.70 & 0.01 & A0 Vae kB8 & $55^{p}$ \\
\hline HD 100740 & HR 4464 & $x$ & 19.6 & 6.57 & 0.13 & A3 IVnn kA2.5mA2.5 & $259^{a}$ \\
\hline HD 101108 & HD 101108 & $\circ$ & 8.1 & 8.89 & 0.15 & A3 IV (4481-wk) & $90^{i}$ \\
\hline HD 101412 & HD 101412 & $\checkmark$ & & 9.29 & 0.18 & A3 Va kA0mA0 $\lambda$ Boo & $3^{q}$ \\
\hline HD 102541 & V* V1023 Cen & $\circ$ & 144.6 & 7.94 & 0.24 & A9 V kA4mA4 ( $\lambda$ Boo $)$ & \\
\hline HD 103483 & $\mathrm{~V} * \mathrm{DN} \mathrm{UMa}$ & $x$ & 10.7 & 6.54 & 0.10 & $\mathrm{kA} 2 \mathrm{hA} 5 \mathrm{~mA} 3 \mathrm{~V}$ & $150^{b}$ \\
\hline HD 105058 & HD 105058 & $\checkmark$ & 7.9 & 8.88 & 0.17 & hA8 V kA0.5mA0.5 $\lambda$ Boo & $130^{e}$ \\
\hline HD 105199 & HD 105199 & $x$ & & 9.83 & 0.14 & kA0.5hF0mA3 V $\lambda$ Boo: & $20^{b}$ \\
\hline HD 105260 & HD 105260 & ? & 78.5 & 9.21 & 0.22 & $\mathrm{hF0mA5} \mathrm{V}$ & \\
\hline HD 105759 & $\mathrm{~V} *$ II Vir & $\checkmark$ & 14.0 & 6.54 & 0.19 & A0 & $120^{h}$ \\
\hline HD 106223 & HD 106223 & $\checkmark$ & 41.4 & 7.43 & 0.26 & $\mathrm{~F} 4 \mathrm{~V}$ kA1.5mA1 $\lambda$ Boo & $80^{g}$ \\
\hline HD 107223 & HD 107223 & $x$ & 35.8 & 8.19 & 0.10 & $\mathrm{~A} 1 \mathrm{IVs}$ & \\
\hline HD 107233 & HD 107233 & $\checkmark$ & 22.7 & 7.36 & 0.24 & kA1hF0mA1 V $\lambda$ Boo & $80^{i}$ \\
\hline HD 108283 & $* 14$ Com & $x$ & 7.0 & 4.92 & 0.26 & A9 IVnp Sr II & $226^{b}$ \\
\hline HD 108714 & HD 108714 & $x$ & 44.0 & 7.74 & 0.09 & A0 & \\
\hline HD 108765 & * $20 \mathrm{Com}$ & $x$ & 16.3 & 5.68 & 0.09 & $\mathrm{kA} 3 \mathrm{hA} 3 \mathrm{~mA} 0 \mathrm{~V}$ & $133^{a}$ \\
\hline HD 109738 & HD 109738 & $\checkmark$ & & 8.30 & 0.20 & A9 $\mathrm{Vn} \mathrm{kB} 9.5 \mathrm{~mA} 0 \lambda$ Boo & $166^{h}$ \\
\hline HD 109980 & $* 9 \mathrm{CVn}$ & $x$ & 9.2 & 6.35 & 0.19 & kA6hA8 Vnn & $250^{b}$ \\
\hline HD 110377 & $\mathrm{~V} * \mathrm{GG}$ Vir & $x$ & 31.7 & 6.22 & 0.19 & A6 Vp $(\lambda$ Boo $)$ & $175^{a}$ \\
\hline HD 110411 & $\mathrm{~V} *$ rho Vir & $\checkmark$ & 20.9 & 4.87 & 0.08 & $\mathrm{kA} 0 \mathrm{hA} 3 \mathrm{~mA} 0$ Va $\lambda$ Boo & $154^{a}$ \\
\hline HD 111005 & HD 111005 & $\circ$ & 32.4 & 7.97 & 0.33 & F2 V kA5mA5 $\lambda$ Boo? & $140^{j}$ \\
\hline HD 111164 & * 34 Vir & $x$ & 19.0 & 6.11 & 0.13 & A3 IV-V & $191^{a}$ \\
\hline HD 111604 & $\mathrm{~V} * \mathrm{DT}$ CVn & $\checkmark$ & 47.6 & 5.89 & 0.16 & kA1.5hA8mA1 Vn $\lambda$ Boo & $200^{b}$ \\
\hline HD 111786 & V* MO Hya & $\checkmark$ & 36.4 & 6.15 & 0.21 & F0 V kA1mA1 $\lambda$ Boo & $47^{c}$ \\
\hline HD 111893 & HR 4886 & $x$ & 23.5 & 6.30 & 0.17 & A5 IV-Vnn & $233^{a}$ \\
\hline HD 112097 & * $41 \mathrm{Vir}$ & $x$ & 21.1 & 6.25 & 0.26 & $\mathrm{kA} 7 \mathrm{hF} 0 \mathrm{mF} 0(\mathrm{~V})$ & $71^{c}$ \\
\hline HD 113848 & * 39 Com & $\times$ & 19.6 & 6.02 & 0.36 & kF0hF8mF3 p & $26^{f}$ \\
\hline HD 114879 & HD 114879 & $x$ & 60.0 & 8.92 & 0.16 & A3 V & \\
\hline HD 114930 & HD 114930 & $x$ & 28.5 & 9.01 & 0.29 & F1 Vs & \\
\hline HD 118623 & CCDM J13375+3617AB & $x$ & 28.4 & 4.82 & 0.23 & kA8hF0 Vnn & $207^{c}$ \\
\hline HD 119288 & G $62-63$ & $x$ & 67.1 & 6.16 & 0.42 & F5 V ((metal-weak)) & $12^{b}$ \\
\hline HD 120500 & $\mathrm{~V} *$ FQ Boo & $\checkmark$ & 21.4 & 6.61 & 0.11 & $\mathrm{kA} 1 / 5 \mathrm{hA} 5 \mathrm{~mA} 1.5 \mathrm{~V}(\lambda$ Boo $)$ & $130^{g}$ \\
\hline HD 120896 & V* QT Vir & $\checkmark$ & 34.4 & 8.51 & 0.25 & kA6hF0mA6 V $\lambda$ Boo & \\
\hline HD 123299 & THUBAN & $x$ & 26.0 & 3.68 & -0.04 & A0 IIIs & $15^{b}$ \\
\hline HD 125162 & LTT 14190 (= $\lambda$ Boötis $)$ & $\checkmark$ & 35.4 & 4.18 & 0.08 & kB9hA3mB9 Va $\lambda$ Boo & $123^{c}$ \\
\hline HD 125489 & HR 5368 & $x$ & 12.1 & 6.19 & 0.19 & A7 Vn & $159^{a}$ \\
\hline HD 125889 & HD 125889 & $\circ$ & & 9.81 & 0.27 & $\mathrm{~F} 1 \mathrm{~V}$ kA4mA4 $(\lambda$ Boo $)$ & \\
\hline HD 128167 & * sig Boo & $x$ & 17.3 & 4.46 & 0.36 & $\mathrm{kF} 2 \mathrm{hF} 4 \mathrm{mF} 1 \mathrm{~V}$ & $5^{b}$ \\
\hline
\end{tabular}


Table 1. Continued

\begin{tabular}{|c|c|c|c|c|c|c|c|}
\hline HD number & SIMBAD identifier & Member? & T. Vel. & $V$ & $B-V$ & Sp.T. & $v \sin i$ \\
\hline HD 130158 & * 55 Hya & $x$ & 16.8 & 5.61 & -0.04 & A0 II-IIIp (Si) & $65^{a}$ \\
\hline HD 130767 & HD 130767 & $\checkmark$ & 28.2 & 6.90 & 0.04 & $\mathrm{~A} 0 \mathrm{Va} \lambda \mathrm{Boo}$ & \\
\hline HD 138527 & * 12 Ser & $\circ$ & 4.8 & 6.22 & -0.04 & B9.5 Vp ( $\lambda$ Boo: $)$ & $158^{a}$ \\
\hline HD 139614 & HD 139614 & $\checkmark$ & & 8.24 & 0.23 & $\mathrm{~A} 7 \mathrm{Ve}$ & $24^{n}$ \\
\hline HD 141569 & HD 141569 & $\checkmark$ & 14.7 & 7.12 & 0.08 & $\mathrm{~A} 2 \mathrm{Ve} \mathrm{kB} 9 \mathrm{mB} 9 \lambda$ Boo & $228^{n}$ \\
\hline HD 141851 & $* \mathrm{~b}$ Ser & $x$ & 22.5 & 5.10 & 0.13 & A2 IVn & $229^{a}$ \\
\hline HD 142666 & HD 142666 & $x$ & & 8.82 & 0.55 & F0 Vs shell? & $65^{n}$ \\
\hline HD 142703 & $\mathrm{~V} * \mathrm{HR} \mathrm{Lib}$ & $\checkmark$ & 19.1 & 6.12 & 0.22 & kA1hF0mA1 Va $\lambda$ Boo & $110^{c}$ \\
\hline HD 142944 & HD 142944 & $x$ & & 10.08 & 0.22 & $\mathrm{~A} 0 \mathrm{~V}$ & \\
\hline HD 142994 & $\mathrm{~V} * \mathrm{IN}$ Lup & $\checkmark$ & & 7.17 & 0.29 & F0 V kA3mA $3 \lambda$ Boo & \\
\hline HD 143148 & HD 143148 & $x$ & & 7.39 & 0.28 & A7 IVn & \\
\hline HD 144708 & $* 11$ Sco & $x$ & 26.3 & 5.76 & 0.01 & B9 $\mathrm{Vp}(\lambda$ Boo $) n n$ & $275^{a}$ \\
\hline HD 145782 & HR 6040 & $x$ & 31.4 & 5.63 & 0.12 & $\mathrm{~A} 3 \mathrm{~V}$ & \\
\hline HD 148638 & $\mathrm{~V} * \mathrm{NP} \operatorname{Tr} \mathrm{A}$ & $?$ & 23.4 & 7.90 & 0.19 & A2 IV $^{-}$n (4481-wk) & $160^{j}$ \\
\hline HD 149130 & HD 149130 & $\circ$ & 21.2 & 8.48 & 0.31 & kA7hF0mA7 V $\lambda$ Boo & \\
\hline HD 149303 & HR 6162 & $?$ & 12.8 & 5.66 & 0.11 & A3 IV-V (4481-wk) + F9 V & $275^{j}$ \\
\hline HD 153747 & V*V922 Sco & $\checkmark$ & 13.6 & 7.42 & 0.12 & A7 V kA0mA0 $\lambda$ Boo & \\
\hline HD 153808 & * eps Her & $x$ & 12.3 & 3.91 & -0.01 & $\mathrm{~A} 0 \mathrm{IV}^{+}$ & $60^{a}$ \\
\hline HD 154153 & HR 6338 & $\circ$ & 9.3 & 6.20 & 0.25 & hF0mA5 ( $\lambda$ Boo) & $125^{c}$ \\
\hline HD 156954 & HD 156954 & $?$ & 18.9 & 7.67 & 0.30 & F1 Vs kA5mA4 & $51^{h}$ \\
\hline HD 159082 & HR 6532 & $x$ & 11.5 & 6.45 & -0.01 & A0p HgMn & $22^{a}$ \\
\hline HD 160928 & HR 6597 & $x$ & 1.9 & 5.88 & 0.14 & $\mathrm{~A} 2 \mathrm{IV}^{-} \mathrm{n}$ & \\
\hline HD 161223 & $\mathrm{~V} * \mathrm{~V} 2314 \mathrm{Oph}$ & $\checkmark$ & & 7.43 & 0.33 & kA5hA9mA5 V ( $\lambda$ Boo $)$ & \\
\hline HD 161868 & * gam Oph & $x$ & 11.7 & 3.75 & 0.04 & kA0hA1mA0 V & $212^{b}$ \\
\hline HD 168740 & V* V346 Pav & $\checkmark$ & 34.5 & 6.13 & 0.19 & A9 V kA2mA2 $\lambda$ Boo & $130^{i}$ \\
\hline HD 168947 & $\mathrm{~V} * \mathrm{~V} 704 \mathrm{CrA}$ & $\checkmark$ & & 8.11 & 0.24 & F0 V kA3mA3 $\lambda$ Boo & \\
\hline HD 169009 & HR 6878 & $x$ & 9.8 & 6.34 & 0.12 & B9 V He-wk & $44^{a}$ \\
\hline HD 169022 & KAUS AUSTRALIS & $x$ & 27.1 & 1.80 & 0.02 & A0 $\mathrm{II}^{-}(\mathrm{n})$ (shell) & $236^{c}$ \\
\hline HD 169142 & HD 169142 & $?$ & & 8.16 & 0.26 & $\mathrm{~F} 1 \mathrm{~V} \mathrm{kA} 4 \mathrm{~mA} 5$ var & $48^{n}$ \\
\hline HD 170000 & V* phi Dra & $x$ & 16.8 & 4.22 & -0.10 & kB9hB9HeA0 V (Si) & $75^{c}$ \\
\hline HD 170680 & HR 6944 & $\widehat{s}$ & 7.5 & 5.13 & 0.01 & A0 Van kB9 ( $\lambda$ Boo $)$ & $222^{a}$ \\
\hline HD 171948 A & HD $171948 \mathrm{~A}$ & $\checkmark$ & 8.1 & 6.77 & 0.05 & $\mathrm{~A} 3 \mathrm{Va}^{-} \mathrm{kB} 8.5 \lambda$ Boo & $15^{k}$ \\
\hline HD $171948 \mathrm{~B}$ & HD $171948 \mathrm{~B}$ & $\checkmark$ & & 11.7 & & & $10^{k}$ \\
\hline HD 172167 & VEGA & $\circ$ & 12.7 & 0.03 & 0.00 & $\mathrm{~A} 0 \mathrm{Va}$ & $5^{b}$ \\
\hline HD 174005 & HD 174005 & $\checkmark$ & 35.5 & 6.50 & 0.22 & A7 V kA2 mA2 $\lambda$ Boo & $87^{c}$ \\
\hline HD 175445 & HD 175445 & $x$ & 12.2 & 7.79 & 0.11 & A1.5 Van & \\
\hline HD 177120 & HD 177120 & $x$ & 9.0 & 6.88 & 0.15 & A0.5 IV (shell) & \\
\hline HD 177756 & * lam Aql & $x$ & 16.7 & 3.43 & -0.08 & B8.5 Vn & $155^{b}$ \\
\hline HD 179218 & HD 179218 & $\circ$ & 25.5 & 7.39 & 0.09 & A0 IVe & $69^{n}$ \\
\hline HD 179791 & HR 7288 & $x$ & 11.4 & 6.48 & 0.09 & A2 IV & $196^{a}$ \\
\hline HD 181470 & HR 7338 & $x$ & 13.7 & 6.26 & 0.00 & B7.5 V + G4 III-IV & $16^{c}$ \\
\hline HD 183007 & HR 7392 & $?$ & 43.5 & 5.71 & 0.19 & A8 Vs kA2(p)mA3 $\lambda$ Boo & \\
\hline HD 183324 & V*V1431 Aql & $\dot{v}$ & 9.5 & 5.79 & 0.08 & kB9hA3mB9 Va $\lambda$ Boo & $110^{a}$ \\
\hline HD 184190 & HD $184190^{\circ}$ & $x$ & & 9.74 & 0.28 & $\mathrm{~A} 7 / 9 \mathrm{~s}$ wl & \\
\hline HD 184779 & HD 184779 & $\checkmark$ & & 8.90 & 0.27 & F0.5 V kA5mA4 ( $\lambda$ Boo $)$ & \\
\hline HD 187949 & V* V505 Sgr & $\times$ & 28.5 & 6.48 & 0.14 & A2 IVn + F8 IV & $101^{b}$ \\
\hline HD 188164 & HR 7588 & $\checkmark$ & 41.1 & 6.38 & 0.15 & A5 IV-V kA2mA3 ( $\lambda$ Boo $)$ & \\
\hline HD 188728 & * phi Aql & $x$ & 10.4 & 5.29 & 0.01 & $\mathrm{~A} 1 \mathrm{~m}$ & $27^{a}$ \\
\hline HD 191850 & HD 191850 & $\checkmark$ & & 9.69 & 0.18 & F0 V kA3mA3 $\lambda$ Boo & \\
\hline HD 192424 & HD 192424 & $x$ & 0.0 & 7.89 & 0.04 & $\mathrm{~A} 2 \mathrm{Vp} \lambda \mathrm{Boo}$ & \\
\hline HD 192640 & V* V1644 Cyg & $\checkmark$ & 19.9 & 4.96 & 0.14 & $\mathrm{kA} 1.5 \mathrm{hA} 7 \mathrm{~mA} 0.5 \lambda$ Boo & $25^{b}$ \\
\hline HD 193063 & IDS $20132+3923$ & $\times$ & 52.3 & 7.73 & -0.05 & B9 III + B9 IIIa & \\
\hline HD 193256 & HD 193256 & $\checkmark$ & & 7.64 & 0.19 & A9 Vn kA2mA2 $\lambda$ Boo & $240^{m}$ \\
\hline HD 193281 & CCDM J20205-2911AB & $?$ & 0.3 & 6.30 & 0.17 & A2 IVn & $95^{a}$ \\
\hline HD 193322 D & HD 193322D & $\times$ & & 11.20 & & $\mathrm{~B} 8 \mathrm{~V} \mathrm{Si}$ & \\
\hline HD 196821 & HR 7903 & $\hat{x}$ & 35.4 & 6.08 & -0.04 & $\mathrm{~A} 0 \mathrm{III}^{+} \mathrm{p}$ kB8mA1 (CP) & $22^{c}$ \\
\hline HD 198160 & HR 7959 & $\hat{\checkmark}$ & & 6.59 & 0.17 & A2 Vann $\lambda$ Boo & $200^{h}$ \\
\hline HD 198161 & HR 7960 & $\checkmark$ & & 6.59 & 0.17 & A3 V & $180^{h}$ \\
\hline HD 200841 & HD 200841 & $x$ & 7.4 & 8.28 & 0.05 & F2 V kA7mA6 $\lambda$ Boo? & \\
\hline HD 201019 & HD 201019 & $?$ & 12.4 & 8.38 & 0.28 & F3 wk met & \\
\hline HD 201184 & * chi Cap & $x$ & 16.5 & 5.28 & 0.01 & $\mathrm{~A} 0 \mathrm{~V}$ & $212^{a}$ \\
\hline HD 204041 & HR 8203 & $\checkmark$ & 17.9 & 6.47 & 0.14 & kA1hA6mA1 V ( $\lambda$ Boo $)$ & $67^{a}$ \\
\hline
\end{tabular}


Table 1. Continued

\begin{tabular}{|c|c|c|c|c|c|c|c|}
\hline HD number & SIMBAD identifier & Member? & T. Vel. & $V$ & $B-V$ & Sp.T. & $v \sin i$ \\
\hline HD 204754 & HR 8226 & $x$ & 38.1 & 6.15 & 0.09 & B5 III-IVs & $19^{c}$ \\
\hline HD 204965 & HR 8237 & $x$ & 20.0 & 6.02 & 0.08 & A2 Vp 4481-wk & $96^{a}$ \\
\hline HD 207978 & * $15 \mathrm{Peg}$ & $x$ & 11.2 & 5.54 & 0.39 & F2 V & $3^{b}$ \\
\hline HD 210111 & HR 8437 & $\checkmark$ & 10.3 & 6.38 & 0.18 & $\mathrm{kA} 2 \mathrm{hA} 7 \mathrm{~mA} 2$ Vas $\lambda$ Boo & $54^{c}$ \\
\hline HD 210418 & * tet Peg & $x$ & 38.1 & 3.50 & 0.11 & hA2mA1 IV-V & $122^{b}$ \\
\hline HD 212061 & * gam Aqr & $x$ & 30.9 & 3.85 & -0.03 & B9.5 III-IV & $75^{b}$ \\
\hline HD 212150 & HR 8525 & $x$ & 17.5 & 6.63 & 0.01 & B9 IIInp kA0 & $196^{a}$ \\
\hline HD 213669 & $\mathrm{~V} * \mathrm{DR}$ Gru & $\circ$ & 16.0 & 7.42 & 0.20 & $\mathrm{~F} 0.5 \mathrm{~V} \mathrm{kA} 2.5 \mathrm{~mA} 2.5 \lambda$ Boo & \\
\hline HD 214454 & * $9 \mathrm{Lac}$ & $x$ & 28.9 & 4.65 & 0.23 & F0 Vp ( $\lambda$ Boo; met A6) & $90^{b}$ \\
\hline HD 216847 & HD 216847 & $\circ$ & 14.3 & 7.06 & 0.23 & $\mathrm{hF0mA3} \mathrm{Vn} \lambda$ Boo & $209^{a}$ \\
\hline HD 217782 & *2 And & $x$ & 34.6 & 5.10 & 0.08 & A2 IIIn & $212^{a}$ \\
\hline HD 218396 & V*V342 Peg & $\checkmark$ & 22.2 & 5.95 & 0.26 & kA5hF0mA5 V $\lambda$ Boo & $49^{a}$ \\
\hline HD 220061 & $\mathrm{~V} *$ tau Peg & $?$ & 7.3 & 4.59 & 0.16 & kA5hA8mA5 V 4481-wk & $150^{b}$ \\
\hline HD 220278 & * 97 Aqr & $?$ & 36.6 & 5.22 & 0.21 & A5 Vn & $175^{a}$ \\
\hline HD 221756 & V*V340 And & $\checkmark$ & 19.0 & 5.56 & 0.09 & $\mathrm{kA} 1 \mathrm{hA} 3 \mathrm{~mA} 0.5 \mathrm{Va}^{+}(\lambda \mathrm{Boo})$ & $86^{c}$ \\
\hline HD 222303 & HD 222303 & ? & & 9.16 & 0.57 & A9 III: (met wk A3) & \\
\hline HD 223352 & $*$ del Scl & $\circ$ & 29.1 & 4.58 & 0.01 & kB9hA0mB9 Van $((\lambda$ Boo $))$ & $299^{a}$ \\
\hline HD 225180 & $* 9$ Cas & $x$ & 7.5 & 5.90 & 0.25 & A3 Vae & $33^{c}$ \\
\hline HD 225218 & ADS 30 & $x$ & 31.1 & 6.12 & 0.15 & A4 IV & $28^{c}$ \\
\hline HD 228509 & HD 228509 & $?$ & & 9.24 & 0.21 & A9 V(n) kA5mA5 & \\
\hline HD 245185 & HD 245185 & $?$ & & 10.00 & 0.03 & A 3 Vae $\mathrm{Bd}<\mathrm{Nem} 1$ & $118^{n}$ \\
\hline HD 261904 & HD 261904 & $?$ & & 10.30 & 0.08 & $\mathrm{~A} 0 \mathrm{Va}^{-}((\lambda \mathrm{Boo}))$ & $150^{g}$ \\
\hline HD 278937 & $\mathrm{~V} *$ IP Per & $\circ$ & & 10.36 & 0.30 & kA3hA7mA4 III:e & $80^{n}$ \\
\hline HD 290492 & CCDM J05313-0029AB & $?$ & & 9.39 & 0.08 & $\mathrm{~A} 0.5 \mathrm{Vb}(\lambda \mathrm{Boo})$ & \\
\hline HD 290799 & $\mathrm{~V} * \mathrm{~V} 1790$ Ori & $\checkmark$ & & 10.80 & 0.00 & $\mathrm{~A} 7 \mathrm{~V} \mathrm{kA} 2 \mathrm{~mA} 2 \lambda \mathrm{Boo}$ & $70^{g}$ \\
\hline HD 294253 & HD 294253 & $\checkmark$ & & 9.67 & 0.01 & A0 Va kB8.5 ( $\lambda$ Boo $)$ & $70^{g}$ \\
\hline TYC $3680-215-1$ & TYC $3680-215-1$ & $\checkmark$ & & 11.00 & 0.50 & $\mathrm{~A} 0 \mathrm{Va}^{-} \lambda \mathrm{Boo}$ & \\
\hline TYC 4774-866-1 & $\mathrm{V} * \mathrm{~T}$ Ori & $?$ & & 11.25 & 0.39 & A3 IVe & $163^{r}$ \\
\hline
\end{tabular}

values, we have checked a sample against The General Catalogue of Photometric Data (Vol. II, Mermilliod et al. 1997). The agreement was satisfactory for almost all the test sample, so for simplicity we adopted the SIMBAD values for every star.

Membership recommendations for inclusion in the $\lambda$ Boo class (fourth column) come from the discussions in the previous section and are given here as follows: $\checkmark$ denotes a member; $\circ$ a probable member; ? an uncertain member; and $x$ a non-member.

Transverse velocities have been calculated from SIMBAD proper motions and parallaxes. These are provided to help eliminate thick disc or Population II objects. One cannot use proper motions alone because nearby stars may have high proper motions yet only small space velocities (e.g. Vega). For stars without parallaxes, no transverse velocity could be calculated. We do, however, recognise the result of Paunzen et al. (2014b), which was that intermediate Pop. II objects cannot be distinguished from $\lambda$ Boo stars by velocities alone. Indeed, we make no attempt to do this, as is evident from the previous section. We do not provide an additional column for the uncertainties on the transverse velocities, but these are provided to be indicative only, and were used with very little weight in our membership evaluations.

The spectral types column contains spectral types as provided in Section 2. When multiple are available, we have cho- sen which is the most appropriate, given the literary sources and the membership. Where possible, we provide our own spectral types based on spectra obtained for this work. Otherwise, we attempt to provide the most trustworthy spectral type from the literature for each object, favouring where possible, those in agreement with the membership recommendations in Section 2. This sometimes results in $\lambda$ Boo classifications for stars we have rejected. We refer the reader to Section 2 in those instances, and strongly recommend cross-referencing our evaluations before quoting any spectral types from Table 1.

Finally, we have provided $v \sin i$ for as many stars as possible, based only on values available in the literature, and we provide a reference for each value as a superscript. Those references can be found in Table 2. For stars without $v \sin i$ values provided, we encourage the community to conduct abundance analyses and to determine $v \sin i$ for the continued investigation of stars belonging the $\lambda$ Boo class.

The number of stars falling in the member, probable member, uncertain member and non-member groups are 64, 19, 26 , and 103, respectively.

\section{ACKNOWLEDGEMENTS}

We thank the referee, Ernst Paunzen, for his thorough reading of this long paper and for his useful comments, and thank 
Table 2. List of references for the $v \sin i$ values in the main table, ordered by most matching entries with Table 1 .

\begin{tabular}{ll}
\hline \hline Note & Reference \\
\hline$a$ & Royer et al. (2007) \\
$b$ & Bernacca \& Perinotto (1970) \\
$c$ & Royer et al. (2002) \\
$d$ & Huang, Gies, \& McSwain (2010) \\
$e$ & Uesugi \& Fukuda (1982) \\
$f$ & Schröder, Reiners \& Schmitt (2009) \\
$g$ & Andrievsky et al. (2002) \\
$h$ & Heiter et al. (2002) \\
$i$ & Heiter (2002) \\
$j$ & Kamp et al. (2001) \\
$k$ & Paunzen et al. (1998a) \\
$m$ & Stürenburg (1993) \\
$n$ & Alecian et al. (2013) \\
$p$ & Acke \& Waelkens (2004) \\
$q$ & Cowley et al. (2010) \\
$r$ & Folsom et al. (2012) \\
$s$ & Rodgers (1968) \\
$t$ & Woolf \& Lambert (1999) \\
$u$ & Kudryavtsev et al. (2007) \\
\hline \hline &
\end{tabular}

Brian Skiff for his attention to detail in noticing some errors in an earlier version. This research was supported by the Australian Research Council. Funding for the Stellar Astrophysics Centre is provided by the Danish National Research Foundation (grant agreement no. DNRF106). The research is supported by the ASTERISK project (ASTERoseismic Investigations with SONG and Kepler) funded by the European Research Council (grant agreement no. 267864). This program is supported by grants from the National Science Foundation to California State University Fullerton (AST1211213), the College of Charleston (AST-1211221), and Appalachian State University (AST-1211215). JEN received additional support through NSFs Independent Research and Development program available to Program Officers.

This research has made extensive use of the SIMBAD data base and the VizieR catalogue access tool (Ochsenbein, Bauer \& Marcout 2000), operated at CDS, Strasbourg, France. Spectral types for some Ap stars were found in the Skiff (2013) Catalogue of Stellar Spectral Classifications, and checked in their original articles. IRAF is distributed by the National Optical Astronomy Observatory, which is operated by the Association of Universities for Research in Astronomy, Inc. under cooperative agreement with the $\mathrm{Na}$ tional Science Foundation.

\section{REFERENCES}

Abt, H. A. 1984a, The MK Process and Stellar Classification, ed. R. F. Garrison (Toronto: University of Toronto, David Dunlap Observatory), 340

Abt, H. A. 1984b, ApJ, 285, 247

Abt, H. A. 1985, ApJS, 59, 95
Abt, H. A. 1988, ApJ, 331, 922

Abt, H. A., \& Cardona, O. 1983, ApJ, 272, 182

Abt, H. A., \& Cardona, O. 1984, ApJ, 276, 266

Abt, H. A., \& Morrell, N. I. 1995, ApJS, 99, 135

Acke, B., van den Ancker, M. E., \& Dullemond, C. P. 2005, A\&A, 436, 209

Acke, B., \& Waelkens, C. 2004, A\&A, 427, 1009

Adelman, S. J., Caliskan, H., Kocer, D., \& Bolcal, C. 1997, MNRAS, 288, 470

Alecian, E., et al. 2013, MNRAS, 429, 1001

Andersen, J., \& Nordstrom, B. 1977, A\&AS, 29, 309

Andrievsky, S. M., Chernyshova, I. V., Usenko, I. A., Kovtyukh, V. V., Panchuk, V. E., \& Galazutdinov, G. A. 1995, PASP, 107, 219

Andrievsky, S. M., et al. 2002, A\&A, 396, 641

Andrillat, Y., Jaschek, C., \& Jaschek, M. 1995, A\&A, 299, 493

Appenzeller, I. 1967, PASP, 79, 102

Balona, L. A. 1977, MmRAS, 84, 101

Barry, D. C. 1970, ApJS, 19, 281

Bartolini, C., Dapergolas, A., Piccioni, A., \& Voli, M. 1980, IBVS, 1757, 1

Baschek, B., Koeppen, J., Scholz, M., Wehrse, R., Heck, A., Jaschek, C., \& Jaschek, M. 1984, A\&A, 131, 378

Baschek, B., \& Slettebak, A. 1988, A\&A, 207, 112

Batten, A. H., Fletcher, J. M., \& Mann, P. J. 1978, PDAO, 15, 121

Bernacca, P. L., Perinotto, M. 1970, CoAsi, 239, 1

Bidelman, W. P. 1943, ApJ, 98, 61

Bidelman, W. P. 1988, PASP, 100, 1084

Bidelman, W. P., Ratcliff, S. J., \& Svolopoulos, S. 1988, PASP, 100, 828

Bohlender, D. A., Gonzalez, J.-F., \& Matthews, J. M. 1999, A\&A, 350,553

Bohlender, D. A., \& Walker, G. A. H. 1994, MNRAS, 266, 891

Booth, M., et al. 2013, MNRAS, 428, 1263

Breger, M. 1969, AJ, 74, 166

Breger, M. 1979, PASP, 91, 5

Breger, M., Hutchins, J., \& Kuhi, L. V. 1976, ApJ, 210, 163

Budaj, J. 1997, A\&A, 326, 655

Cayrel de Strobel, G., Soubiran, C., Friel, E. D., Ralite, N., \& Francois, P. 1997, A\&AS, 124, 299

Cenarro, A. J., et al. 2007, MNRAS, 374, 664

Cheng, K.-P., Bruhweiler, F. C., Kondo, Y., \& Grady, C. A. 1992, ApJ, 396, L83

Childress, M. J., Vogt, F. P. A., Nielsen, J., \& Sharp, R. G. 2014, Ap\&SS, 349, 617

Colomba, A., de Benedetto, G., \& Ielo, A. 1991, IBVS, 3597, 1

Corbally, C. J. 1984, ApJS, 55, 657

Corbally, C. J., \& Garrison, R. F. 1980, PASP, 92, 493

Corbally, C. J., \& Gray, R. O. 1996, AJ, 112, 2286

Cowley, A., Cowley, C., Jaschek, M., \& Jaschek, C. 1969, AJ, 74, 375

Cowley, A. P., \& Bidelman, W. P. 1979, PASP, 91, 83

Cowley, C. R. 1991, in IAU Symp., 145, Evolution of Stars: the Photospheric Abundance Connection, ed. G. Michaud, \& A. V. Tutukov (Dordrecht: Kluwer), 183

Cowley, C. R., Hubrig, S., González, J. F., \& Savanov, I. 2010, A\&A, 523, A65

Crawford, D. L. 1975, AJ, 80, 955

Crawford, D. L. 1978, AJ, 83, 48

Crawford, D. L. 1979, AJ, 84, 1858 
Cucchiaro, A., Jaschek, M., Jaschek, C., \& Macau-Hercot, D. 1980, A\&AS, 40, 207

Cucchiaro, A., Macau-Hercot, D., Jaschek, M., \& Jaschek, C. 1979, A\&AS, 35, 75

Desikachary, K., \& McInally, C. J. 1979, MNRAS, 188, 67

Dommanget, J., \& Nys, O. 2002, yCat, 1274, 0

Dopita, M., Hart, J., McGregor, P., Oates, P., Bloxham, G., \& Jones, D. 2007, Ap\&SS, 310, 255

Dunkin, S. K., Barlow, M. J., \& Ryan, S. G. 1997, MNRAS, 286, 604

Eggen, O. J. 1984, AJ, 89, 1878

Eggleton, P. P., \& Tokovinin, A. A. 2008, MNRAS, 389, 869

Erspamer, D., \& North, P. 2003, A\&A, 398, 1121

Faraggiana, R. 1990, in IUE Proposal, 3832, IUE Proposal ID \#LA061

Faraggiana, R., \& Bonifacio, P. 1999, A\&A, 349, 521

Faraggiana, R., \& Bonifacio, P. 2005, A\&A, 436, 697

Faraggiana, R., Bonifacio, P., Caffau, E., Gerbaldi, M., \& Nonino, M. 2004, A\&A, 425, 615

Faraggiana, R., \& Gerbaldi, M. 2003, A\&A, 398, 697

Faraggiana, R., Gerbaldi, M., \& Boehm, C. 1990, A\&A, 235, 311

Faraggiana, R., Gerbaldi, M., \& Bonifacio, P. 2001a, A\&A, 380, 286

Faraggiana, R., Gerbaldi, M., Bonifacio, P., \& François, P. 2001b, A\&A, 376, 586

Faraggiana, R., Gerbaldi, M., \& Burnage, R. 1997, A\&A, 318, L21

Feigelson, E. D., Lawson, W. A., \& Garmire, G. P. 2003, ApJ, 599, 1207

Folsom, C. P., Bagnulo, S., Wade, G. A., Alecian, E., Landstreet, J. D., Marsden, S. C., \& Waite, I. A. 2012, MNRAS, 422, 2072

Garrison, R. F., \& Gray, R. O. 1994, AJ, 107, 1556

Gerbaldi, M., \& Faraggiana, R. 1993, in ASP Conf. Ser., 44, IAU Colloq. 138: Peculiar versus Normal Phenomena in A-type and Related Stars, ed. M. M. Dworetsky, F. Castelli, R. Faraggiana (San Francisco: Astron. Soc. Pac.), 368

Gerbaldi, M., \& Faraggiana, R. 2004, in ASP Conf. Ser., 318, Spectroscopically and Spatially Resolving the Components of the Close Binary Stars, ed. R. W. Hilditch, H. Hensberge, K. Pavlovski (San Francisco: Astron. Soc. Pac.), 312

Gerbaldi, M., Faraggiana, R., \& Lai, O. 2003, A\&A, 412, 447

Glagolevskij, Y. V. 2011, Ap, 54, 231

Gopka, V., et al. 2007, in ASP Conf. Ser., 362, The $7^{\text {th }}$ Pacific Rim Conference on Stellar Astrophysics, ed. Y. W. Kang, H.-W. Lee, K.-C. Leung, \& K.-S. Cheng (San Francisco: Astron. Soc. Pac.), 249

Graham, J. A., \& Slettebak, A. 1973, AJ, 78, 295

Gray, R. O. 1986, PhD thesis, University of Toronto.

Gray, R. O. 1988, AJ, 95, 220

Gray, R. O. 1989, AJ, 98, 1049

Gray, R. O. 2014, in Determination of Atmospheric Parameters of B-, A-, F- and G-Type Stars, E. Niemczura, B. Smalley, W. Pych (Switzerland: Springer International Publishing), 75

Gray, R. O., \& Corbally, C. J. 1993, AJ, 106, 632

Gray, R. O., \& Corbally, C. J. 1998, AJ, 116, 2530

Gray, R. O., \& Corbally, C. J. 2002, AJ, 124, 989

Gray, R. O., \& Corbally, C. J. 2014, AJ, 147, 80

Gray, R. O., Corbally, C. J., Garrison, R. F., McFadden, M. T., \& Robinson, P. E. 2003, AJ, 126, 2048

Gray, R. O., Corbally, C. J., \& Philip, A. G. D. 1996, AJ, 112, 2291

Gray, R. O., \& Corbally, J. C. 2009, Stellar Spectral Classification (Princeton, NJ: Princeton University Press)
Gray, R. O., \& Garrison, R. F. 1987, ApJS, 65, 581

Gray, R. O., \& Garrison, R. F. 1989a, ApJS, 69, 301

Gray, R. O., \& Garrison, R. F. 1989b, ApJS, 70, 623

Gray, R. O., \& Kaye, A. B. 1999, AJ, 118, 2993

Gray, R. O., Napier, M. G., \& Winkler, L. I. 2001, AJ, 121, 2148

Gray, R. O., \& Olsen, E. H. 1991, A\&AS, 87, 541

Grenier, S., Burnage, R., Faraggiana, R., Gerbaldi, M., Delmas, F., Gómez, A. E., Sabas, V., \& Sharif, L. 1999, A\&AS, 135, 503

Griffin, R. E., Gray, R. O., \& Corbally, C. J. 2012, A\&A, 547, A8

Guetter, H. H. 1981, AJ, 86, 1057

Handler, G. 1999, IBVS, 4817, 1

Handler, G., Gray, R. O., \& Shobbrook, R. R. 2000, IBVS, 4876, 1

Hashimoto, J., et al. 2011, ApJ, 729, L17

Hauck, B. 1986, A\&A, 154, 349

Hauck, B., Ballereau, D., \& Chauville, J. 1998, A\&AS, 128, 429

Hauck, B., \& Mermilliod, M. 1998, A\&AS, 129, 431

Hauck, B., \& Slettebak, A. 1983, A\&A, 127, 231

Heiter, U. 1998, CoSka, 27, 403

Heiter, U. 2002, A\&A, 381, 959

Heiter, U., Kupka, F., Paunzen, E., Weiss, W. W., \& Gelbmann, M. 1998, A\&A, 335, 1009

Heiter, U., Weiss, W. W., \& Paunzen, E. 2002, A\&A, 381, 971

Hekker, S., Frémat, Y., Lampens, P., De Cat, P., Niemczura, E., Creevey, O. L., \& Zorec, J. 2009, MNRAS, 396, 1689

Heller, C. H., \& Kramer, K. S. 1988, PASP, 100, 583

Hoffleit, D., \& Jaschek, C. 1982, The Bright Star Catalogue, 4th revised edn. (containing data compiled through 1979) (New Haven: Yale University Observatory)

Holweger, H., \& Rentzsch-Holm, I. 1995, A\&A, 303, 819

Holweger, H., \& Stuerenburg, S. 1991, A\&A, 252, 255

Horn, J., Kubat, J., Harmanec, P., Koubsky, P., Hadrava, P., Simon, V., Stefl, S., \& Skoda, P. 1996, A\&A, 309, 521

Houk, N. 1978, Michigan Catalogue of Two-Dimensional Spectral Types for the HD Stars (Ann Arbor, MI: University of Michigan)

Houk, N. 1982, Michigan Catalogue of Two-dimensional Spectral Types for the HD Stars, Volume 3, Declinations $-40^{\circ}$ to $-26^{\circ}$ (Ann Arbor, MI: University of Michigan)

Houk, N., \& Cowley, A. P. 1975, University of Michigan Catalogue of two-dimensional spectral types for the HD stars, Volume 1 , Declinations $-90^{\circ}$ to $-53^{\circ}$ (Ann Arbor, MI: University of Michigan)

Houk, N., \& Smith-Moore, M. 1988, Michigan Catalogue of Twodimensional Spectral Types for the HD Stars, Volume 4, Declinations $-26^{\circ}$ to $-12^{\circ}$ (Ann Arbor, MI: University of Michigan)

Huang, W., Gies, D. R., \& McSwain, M. V. 2010, ApJ, 722, 605

Hube, D. P. 1970, MmRAS, 72, 233

Ibanoğlu, C., Soydugan, F., Soydugan, E., \& Dervişoğlu, A. 2006, MNRAS, 373, 435

Iliev, I. K., \& Barzova, I. S. 1993a, in ASP Conf. Ser., 44, IAU Colloq. 138: Peculiar versus Normal Phenomena in A-type and Related Stars, ed. M.M. Dworetsky, F. Castelli, R. Faraggiana (San Francisco: Astron. Soc. Pac.), 423

Iliev, I. K., \& Barzova, I. S. 1993b, Ap\&SS, 208, 277

Iliev, I. K., \& Barzova, I. S. 1995, A\&A, 302, 735

Iliev, I. K., \& Barzova, I. S. 1998, CoSka, 27, 441

Iliev, I. K., Paunzen, E., Barzova, I., Andrievsky, S. M., Chernyshova, I. V., \& Kamp, I. 2001, IBVS, 5178, 1

Iliev, I. K., Paunzen, E., Barzova, I. S., Griffin, R. F., Kamp, I., Claret, A., \& Koen, C. 2002, A\&A, 381, 914 
Ilijic, S., Rosandic, M., Dominis, D., Planinic, M., \& Pavlovski, K. 1998, CoSka, 27, 467

Jackisch, G. 1972, AN, 294, 1

Jaschek, M., \& Jaschek, C. 1980, A\&AS, 42, 115

Kamp, I., Iliev, I. K., Paunzen, E., Pintado, O. I., Solano, E., \& Barzova, I. S. 2001, A\&A, 375, 899

Kamp, I., \& Paunzen, E. 2002, MNRAS, 335, L45

King, J. R. 1994, MNRAS, 269, 209

Koen, C., Kilkenny, D., van Wyk, F., Roberts, G., \& Marang, F. 1995, MNRAS, 277, 217

Koen, C., Paunzen, E., van Wyk, F., Marang, F., Chernyshova, I. V., Andrievsky, S. M. 2003, MNRAS, 338, 931

Kohoutek, L. 2001, A\&A, 378, 843

Kudryavtsev, D. O., Romanyuk, I. I., Elkin, V. G., \& Paunzen, E. 2006, MNRAS, 372, 1804

Kudryavtsev, D. O., Romanyuk, I. I., Semenko, E. A., \& Solov'ev, G. A. 2007, AstBu, 62, 147

Kuschnig, R., Gelbmann, M., Paunzen, E., \& Weiss, W. W. 1996 IBVS, 4310, 1

Kuschnig, R., Paunzen, E., \& Weiss, W. W. 1994a, IBVS, 4069, 1

Kuschnig, R., Paunzen, E., \& Weiss, W. W. 1994b, IBVS, 4070, 1

Kuschnig, R., Paunzen, E., \& Weiss, W. W. 1997, IBVS, 4483, 1

Landstreet, J. D., Borra, E. F., Angel, J. R. P., \& Illing, R. M. E. 1975, ApJ, 201, 624

Lemke, M. 1989, A\&A, 225, 125

Liu, N., et al. 1997, ApJ, 485, 350

Lloyd, C. 1981, MNRAS, 195, 805

Maitzen, H. M., \& Pavlovski, K. 1989a, A\&AS, 81, 335

Maitzen, H. M., \& Pavlovski, K. 1989b, A\&A, 219, 253

Marchetti, E., Faraggiana, R., \& Bonifacio, P. 2001, A\&A, 370, 524

Martinez, P., Koen, C., Handler, G., \& Paunzen, E. 1998, MNRAS, 301, 1099

Mason, B. D., Wycoff, G. L., Hartkopf, W. I., Douglass, G. G., Worley, C. E. 2001, AJ, 122, 3466

Matthews, J. M., Wehlau, W. H. 1985, IBVS, 2725, 1

Mermilliod, J.-C., Mermilliod, M., \& Hauck, B. 1997, A\&AS, 124, 349

Mora, A., et al. 2001, A\&A, 378, 116

Morgan, W. W., Keenan, P. C., \& Kellman, E. 1943, An Atlas of Stellar Spectra, with an Outline of Spectral Classification (Chicago, IL: University of Chicago Press)

Moya, A., Amado, P. J., Barrado, D., García Hernández, A., Aberasturi, M., Montesinos, B., \& Aceituno, F. 2010, MNRAS, 405, L81

Mulders, G. D., Paardekooper, S.-J., Panić, O., Dominik, C., van Boekel, R., \& Ratzka, T. 2013, A\&A, 557, A68

Murphy, S. J. 2014, PhD thesis, Jeremiah Horrocks Institute, University of Central Lancashire

Murphy, S. J., et al. 2013, MNRAS, 432, 2284

Nakos, T., Sinachopoulos, D., \& van Dessel, E. 1995, A\&AS, 112 , 453

Narusawa, S.-Y., Ozaki, S., Kambe, E., \& Sadakane, K. 2006a, PASJ, 58, 617

Narusawa, S.-Y., Ozaki, S., Okyudo, M., Takano, R., \& Nakamura, Y. 2006b, PASP, 118, 809

Nordström, B., et al. 2004, A\&A, 418, 989

Oblak, E. 1978, A\&AS, 34, 453

Ochsenbein, F., Bauer, P., \& Marcout, J. 2000, A\&AS, 143, 23

Ohanesyan, J. B. 2008, Ap, 51, 490

Olsen, E. H. 1979, A\&AS, 37, 367

Olsen, E. H. 1980, A\&AS, 39, 205
Olsen, E. H. 1983, A\&AS, 54, 55

Oppenheimer, B. R., et al. 2008, ApJ, 679, 1574

Osawa, K. 1965, AnTok, 9, 121

Parenago, P. P. 1958, SvA, 2, 151

Paunzen, E. 2000, PhD thesis, University of Vienna

Paunzen, E. 2001, A\&A, 373, 633

Paunzen, E., \& Gray, R. O. 1997, A\&AS, 126, 407

Paunzen, E., \& Handler, G. 1996, IBVS, 4318, 1

Paunzen, E., Heiter, U., Handler, G., Garrido, R., Solano, E., Weiss, W. W., \& Gelbmann, M. 1998a, A\&A, 329, 155

Paunzen, E., Weiss, W. W., Heiter, U., \& North, P. 1997, A\&AS, 123, 93

Paunzen, E., Weiss, W. W., \& Kuschnig, R. 1996, IBVS, 4302, 1

Paunzen, E., Weiss, W. W., Martinez, P., Matthews, J. M., Pamyatnykh, A. A., \& Kuschnig, R. 1998b, A\&A, 330, 605

Paunzen, E., Weiss, W. W., \& North, P. 1994, IBVS, 4068, 1

Paunzen, E., et al. 1998c, A\&A, 335, 533

Paunzen, E., Andrievsky, S. M., Chernyshova, I. V., Klochkova, V. G., Panchuk, V. E., \& Handler, G. 1999a, A\&A, 351, 981

Paunzen, E., Duffee, B., Heiter, U., Kuschnig, R., \& Weiss, W. W. 2001, A\&A, 373, 625

Paunzen, E., Heiter, U., Fraga, L., \& Pintado, O. 2012, MNRAS, 419,3604

Paunzen, E., Iliev, I. K., Fossati, L., Heiter, U., \& Weiss, W. W. 2014b, A\&A, 567, A67

Paunzen, E., Iliev, I. K., Kamp, I., \& Barzova, I. S. 2002a, MNRAS, 336, 1030

Paunzen, E., Kamp, I., Weiss, W. W., \& Wiesemeyer, H. 2003, A\&A, 404, 579

Paunzen, E., Skarka, M., Holdsworth, D. L., Smalley, B., \& West, R. G. 2014a, MNRAS, 440, 1020

Paunzen, E., Stütz, C., \& Maitzen, H. M. 2005, A\&A, 441, 631

Paunzen, E., et al. 1999b, A\&A, 345, 597

Paunzen, E., et al. 2002b, A\&A, 392, 515

Pavlovski, K., Schnell, A., \& Maitzen, H. M. 1993, in ASP Conf. Ser., 44, IAU Colloq. 138: Peculiar versus Normal Phenomena in A-type and Related Stars, ed. M. M. Dworetsky, F. Castelli, \& R. Faraggiana (San Francisco: Astron. Soc. Pac.), 429

Philip, A. G. D., \& Hayes, D. S. 1983, ApJS, 53, 751

Renson, P., \& Manfroid, J. 2009, A\&A, 498, 961

Renson, P., Faraggiana, R., \& Boehm, C. 1990, BICDS, 38, 137

Richards, M. T., \& Albright, G. E. 1999, ApJS, 123, 537

Rodgers, A. W. 1968, ApJ, 152, 109

Rodríguez, E., \& Breger, M. 2001, A\&A, 366, 178

Rodríguez, E., López-González, M. J., \& López de Coca, P. 2000, A\&AS, 144, 469

Royer, F., Grenier, S., Baylac, M.-O., Gómez, A. E., \& Zorec, J. 2002, A\&A, 393, 897

Royer, F., Zorec, J., \& Gómez, A. E. 2007, A\&A, 463, 671

Sadakane, K., \& Nishida, M. 1986, PASP, 98, 685

Saffe, C., Gómez, M., Pintado, O., \& González, E. 2008, A\&A, 490, 297

Sargent, W. L. W. 1965, ApJ, 142, 787

Schröder, C., Reiners, A., \& Schmitt, J. H. M. M. 2009, A\&A, 493, 1099

Shobbrook, R. R. 2005, JAD, 11, 7

Skarka, M. 2013, A\&A, 549, A101

Skiff, B. A. 2013, yCat, 1, 2023

Slawson, R. W., Hill, R. J., \& Landstreet, J. D. 1992, ApJS, 82, 117

Slettebak, A. 1952, ApJ, 115, 575

Slettebak, A. 1954, ApJ, 119, 146 
Slettebak, A. 1963, ApJ, 138, 118

Slettebak, A. 1968, ApJ, 154, 933

Slettebak, A. 1975, ApJ, 197, 137

Slettebak, A., Wright, R. R., \& Graham, J. A. 1968, AJ, 73, 152

Sódor, Á., et al. 2014, A\&A, 568, A106

Solano, E., \& Paunzen, E. 1998, in ESA Special Publication, ed. W. Wamsteker, R. Gonzalez Riestra, \& B. Harris, Vol. 413, Ultraviolet Astrophysics Beyond the IUE Final Archive (Noordwijk: ESA), 129

Solano, E., \& Paunzen, E. 1999, A\&A, 348, 825

Solano, E., Paunzen, E., Pintado, O. I., \& Córdoba Varela, J. 2001, A\&A, 374, 957

Soubiran, C., Le Campion, J.-F., Cayrel de Strobel, G., \& Caillo, A. 2010, A\&A, 515, A111

Soummer, R., Brendan Hagan, J., Pueyo, L., Thormann, A., Rajan, A., \& Marois, C. 2011, ApJ, 741, 55

Stürenburg, S. 1993, A\&A, 277, 139

Stütz, C., \& Paunzen, E. 2006, A\&A, 458, L17,

Szczygieł, D. M., \& Fabrycky, D. C. 2007, MNRAS, 377, 1263
Takeda, Y., \& Sadakane, K. 1997, PASJ, 49, 571

Uesugi, A., \& Fukuda, I. 1982, Catalogue of Stellar Rotational Velocities (revised) (Kyoto, Japan: Kyoto University, Department of Astronomy)

Venn, K. A., \& Lambert, D. L. 1990, ApJ, 363, 234

Vican, L. 2012, AJ, 143, 135

Vogt, N., Kerschbaum, F., Maitzen, H. M., \& Faundez-Abans, M. 1998, A\&AS, 130, 455

Warren, Jr W. H., \& Hoffleit, D. 1987, BAAS 19, 733

Weiss, W. W., Paunzen, E., Kuschnig, R., \& Schneider, H. 1994, A\&A, 281, 797

Woolf, V. M., Lambert, D. L. 1999, ApJ, 520, L55

Wraight, K. T., Fossati, L., Netopil, M., Paunzen, E., Rode-Paunzen, M., Bewsher, D., Norton, A. J., \& White, G. J. 2012, MNRAS, 420, 757

Wright, D. J., et al. 2011, ApJ, 728, L20

Zasche, P., Uhlář, R., Šlechta, M., Wolf, M., Harmanec, P., Nemravová, J. A., \& Korčáková, D. 2012, A\&A, 542, A78

Zverko, J., Žižňovský, J., Mikulášek, Z., \& Iliev, I. K. 2008, CoSka, 38,467 